\title{
Local profiles for elliptic problems at different scales: defects in, and interfaces between periodic structures.
}

\author{
X. Blanc ${ }^{1}$, C. Le Bris ${ }^{2} \&$ P.-L. Lions ${ }^{3}$ \\ ${ }^{1}$ Université Paris Diderot, Laboratoire Jacques-Louis Lions, \\ Bâtiment Sophie Germain, 5, rue Thomas Mann \\ 75205 Paris Cedex 13, FRANCE, \\ blanc@ann.jussieu.fr \\ 2 Ecole des Ponts and INRIA, \\ $6 \& 8$, avenue Blaise Pascal, 77455 Marne-La-Vallée Cedex 2, FRANCE \\ lebris@cermics.enpc.fr \\ ${ }^{3}$ Collège de France, 11, place Marcelin Berthelot, \\ 75231 Paris Cedex 05, and \\ CEREMADE, Université Paris Dauphine, Place de Lattre de Tassigny, \\ 75775 Paris Cedex 16, FRANCE \\ lions@ceremade.dauphine.fr
}

April 16, 2015

\begin{abstract}
Following-up on our previous work [10], we present a general approach to approximate at the fine scale the solution to an elliptic equation with oscillatory coefficient when this coefficient consists of a "nice" (in the simplest possible case say periodic) function which is, in some sense to be made precise, perturbed. The approach is based on the determination of a local profile, solution to an equation similar to the corrector equation in classical homogenization. The well-posedness of that equation, in various functional settings depending upon the nature of the perturbation, is the purpose of this article. The case of a local perturbation is first addressed. The case of a more complex geometrical structure (such as the prototypical case of two different periodic structures separated by a common interface) is next discussed. Some related problems, and future directions of research are mentioned. Most of the results presented here have been announced in [28] and summarized in [11].
\end{abstract}

Keywords: Homogenization; elliptic PDE; defects; quasiperiodic; interface

AMS subject classification: 35J15, 35J70, 35B27, 74Q15, 76M50 


\section{Contents}

1 Introduction 3

2 Homogenization setting and previous works 5

2.1 Homogenization setting . . . . . . . . . . . . . . . . . . . . . 5

2.2 The $L^{2}$ case . . . . . . . . . . . . . . . . . . . . . . . . . . . . . . . . 7

3 Local defect in $L^{r}, 1 \leq r<d \quad 10$

4 Local defect in $L^{r}, r<+\infty \quad 12$

5 Interfaces between periodic structures 18

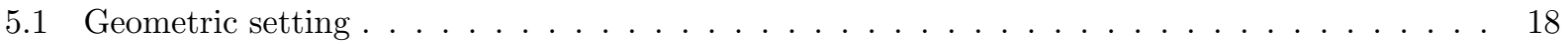

5.2 Matching interfaces . . . . . . . . . . . . . . . . . . . . . . . . 19

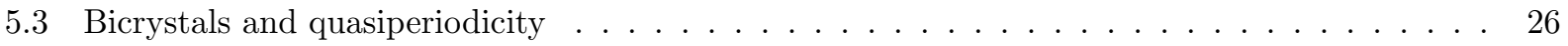

5.4 Remarks on the corrector in quasiperiodic homogenization . . . . . . . . . . . . . . 40

6 Related issues and future works 42

A Technical lemmas 


\section{Introduction}

Our motivation for the mathematical theory we develop here stems from our interest in a particular class of multiscale problems for which we aim to establish results similar to those of classical homogenization theory for the approximation of the oscillating solution. The specifics of the problems under consideration is that these problems present, at the small scale, a geometry that is typically a "nice" geometry (that is, amenable to classical techniques of homogenization, and, for simplicity of exposition, we first choose it periodic here) perturbed by a "local" (in a sense to be made precise below) modification of the structure, namely a defect. Even in the simple case where this defect does not modify the macroscopic behavior, that is, the homogenized solution, it may nevertheless affect the solution locally, and at the small scale. There is therefore some definite interest in examining the behavior of the solution at the vicinity of this defect. We indeed note that, in many practical applications, the interest of practitioners primarily lies in the understanding of the impact of the presence of defects (think of cracks, grain boundaries, dislocations, point defects in materials) onto the response of the medium under study. Our purpose is both theoretical and computational. The present contribution only contains theoretical considerations. We hope to complement those considerations with some numerical experiments further demonstrating the interest of the approach in the future. Note however the numerical simulations already reported on in [10].

Our starting point is a simple scalar elliptic equation in divergence form:

$$
-\operatorname{div}\left(a(x / \varepsilon) \nabla u^{\varepsilon}\right)=f
$$

supplied with homogeneous Dirichlet boundary conditions on a bounded regular domain $\Omega \subset \mathbb{R}^{d}, d \geq 1$, a right-hand-side term $f \in L^{2}(\Omega)$ and where the coefficient $a$ (see more details below) is such that the above problem is well-posed, for $\varepsilon>0$ fixed, by classical arguments. We readily mention that, throughout this article, the coefficient is assumed scalar-valued for simplicity. The extension to a matrix-valued coefficient is only a matter of technicalities. When the matrix is symmetric, the extension is immediate and we will not address this extension at all. When the matrix is not symmetric, some slight modifications are occasionally in order. We also note that other equations, such as fully nonlinear equations, will be briefly discussed toward the end of the present contribution. Their study is postponed until [29] and forthcoming works by the same authors.

The well-known difficulty in (1) is that, when the coefficient $\varepsilon$ encoding the existence of two different scales in the original problem is small, the solution $u^{\varepsilon}$ oscillates wildly and is therefore delicate, if not impossible, to capture by traditional discretization techniques. Homogenization theory provides a way to approximate the solution $u^{\varepsilon}$, for $\varepsilon$ small. We refer the reader to [5, 22] for an exposition of this classical theory. The theoretical developments of homogenization are also useful, as guidelines, to construct efficient numerical approaches, see for instance [15]. The bottom line for the efficiency of homogenization-based approaches is that, when the problem (1) is to be solved repeatedly, then it is useful to design a numerical approach that, although perhaps too costly for a single computation, scales much better in terms of computational time when several $f$ in (1) are successively considered. This may occur if the solution is needed for a large number of right-hand-sides $f$, a context called multi-query context by the practitioners. Another possible situation is the case where only one right-hand-side $f$ is considered, but the domain is so large (or the mesh so fine) that a domain decomposition is used. Then, in the iterations due to this numerical strategy, the problem is solved repeatedly for each subdomain. Our practical motivation for the theoretical developments performed here is exactly in the same vein.

The practical efficiency mentioned above is typically achieved, for simple, say periodic coefficients $a=a_{\text {per }}$ in (1), upon considering the corrector problem

$$
-\operatorname{div}\left(a_{p e r}(x)\left(p+\nabla w_{p, p e r}(x)\right)\right)=0, \quad w_{p, p e r} \text { is periodic, }
$$

posed for each fixed vector $p \in \mathbb{R}^{d}$ and associated to (1). Its periodic solution $w_{p, p e r}$ provides a local model for the oscillations of the solution $u^{\varepsilon}$ to (1). At the theoretical level, this is encoded in the two-scale approximation of $u^{\varepsilon}$ :

$$
u^{\varepsilon}=u^{*}(x)+\varepsilon \sum_{i=1}^{d} \partial_{x_{i}} u^{*}(x) w_{e_{i}, p e r}(x / \varepsilon)+\text { h.o.t. }
$$


where $u^{*}$ denotes the homogenized limit of $u^{\varepsilon}$, that is the solution to

$$
-\operatorname{div}\left(A^{*} \nabla u^{*}\right)=f
$$

where $A^{*}$ is the homogenized coefficient (actually also computed from local averages of the solution $w_{p, p e r}$ to (2)). See more details on the classical theory in Section 2 below.

At the practical, computational level, one e.g. uses equation (2), or equations similar in spirit to that equation, to construct finite element basis functions which better mimic, thus better capture the local oscillations of $u^{\varepsilon}$. They therefore allow for a more efficient approximation of the solution than generic basis functions.

Interestingly, there are some cases (and the periodic context is one such case) when the quality of approximation provided by (3) extends to the microscopic scale (that is, when $x$ is replaced by $\varepsilon x$ ). Put differently, problem (2) is therefore a key step in the understanding, and approximation, of the solution $u^{\varepsilon}$ both locally and globally. This fact is intuitively clear when one has realized that problem (2) is obtained zooming-in from (1) to the small scale.

Our intent is to extend the above approximation technique and properties to the context where the coefficient $a$, encoding in (1) the intrinsic geometric structure of the medium (or material, in the computational mechanics terminology) considered, is a perturbation, in a sense to be made precise, of a simple geometry. The two instances explicitly considered in the present manuscript are:

- a local perturbation of a periodic structure,

- a juxtaposition of two different periodic structures along a common interface,

the terms local perturbation and juxtaposition being made precise mathematically in the sequel.

The above discussion on the periodic case suggests, and this is indeed the case, that a fundamental step in our endeavor is the consideration of the problem analogous to (2) in each of our specific contexts. If $a$ in (1) denotes the coefficient modeling our medium ( $a$ will be made precise below), this problem reads, for each $p \in \mathbb{R}^{d}$,

$$
\left\{\begin{array}{l}
-\operatorname{div}\left(a\left(p+\nabla w_{p}\right)\right)=0 \quad \text { in } \mathbb{R}^{d}, \\
\frac{w_{p}(x)}{1+|x|} \stackrel{|x| \rightarrow \infty}{\longrightarrow} 0,
\end{array}\right.
$$

where, evidently, the strict sub-linearity condition (5.2) is the generalization of the periodicity condition imposed on $w_{p, p e r}$ in the simple setting (2). The reader familiar with homogenization theory is well aware that this strict sub-linearity is in fact the key property useful when considering the approximation of $u^{\varepsilon}$ from (3), in $H^{1}(\Omega)$. Formally, the reader unfamiliar with the topic of homogenization observes on (3) that $u^{\varepsilon}-u^{*}$ can converge to zero as $\varepsilon$ vanishes only if this strict-subadditivity holds.

The study of the well-posedness of problem (5) is the main purpose of the present work.

Our contribution is organized as follows.

Section 2 formalizes mathematically the somewhat vague description we have given above. In particular, it briefly reviews our previous work [10] where the case of a perturbation in $L^{2}\left(\mathbb{R}^{d}\right)$ of a periodic coefficient was considered. This Hilbertian case $r=2$ has been addressed using the Lax-Milgram Theorem and a regularization. Our discussion of that section is also the opportunity to recall, to the attention of the reader not familiar with homogenization theory, some basic facts, and to expand upon the approximation property at the small scale which we alluded to above.

We then proceed with the generalization of our result of [10] to the case of a perturbation in $L^{r}\left(\mathbb{R}^{d}\right)$, for $1 \leq r<+\infty$, the exponent $r$ being not necessarily equal to 2 . Section 3 addresses the case $r<d$, while Section 4 considers the more general case $1 \leq r<+\infty$, upon some additional assumptions. In Section 3, the case $r<d$ is understood using a representation of the solution and the classical Marcinkiewitz estimates on the Green function of the operator $-\operatorname{div}(a \nabla$.). It is then easily proven that the solution to (5) exists, as a superposition of the periodic classical corrector and a function that vanishes at infinity. The uniqueness, up to an additive constant of course, is a consequence of a classical Liouville-type Theorem. Interestingly, the 
proof does not make use of the periodicity of the unperturbed coefficient and therefore carries over to some more general cases. In Section 4, the more delicate case $r>d$ is addressed with a proof (actually essentially valid also for $r \leq d$ ) that, in its present state, requires the additional assumption that the coefficient $a$ is sufficiently regular. The proof is more intricate than when $r<d$ and involves i) estimations of the Green function in dyadic rings, somewhat in the spirit of the Littlewood-Payley decomposition and ii) the classical result [3] on the Green function of the periodic operator - div $\left(a_{p e r} \nabla\right.$.). We construct a solution $w_{p}$ to (5), the gradient of which consists of the superposition of the gradient of the periodic corrector and a gradient in $L^{r}$. The strict sub-linearity (5.2) follows by application of the Sobolev inequalities which actually show Hölder continuity. Uniqueness is obtained by a separate argument. We are unfortunately unable to conclude on the general case of a perturbation that only goes to zero at infinity, i.e. the case $r=\infty$.

In Section 5, we consider a different setting, consisting of two different periodic structures separated by a common interface. This setting can already be seen as a "defect", which in some sense, is unbounded in $d-1$ directions, and thus intrinsically different in nature from the local defect considered in the previous sections. We may superimpose to that defect another, more local defect. The setting of Section 5 raises new questions. In the particular case, considered in Subsection 5.2, when the two periodic structures match conveniently, the technicalities are limited and the approach of the previous sections designed for a local perturbation carries over to this case with not much additional difficulty. When the structures do not match conveniently, some quasiperiodic feature arises along the interface and the situation readily gets more complicated. We specifically address this case in Subsection 5.3. Subsection 5.4 contains some remarks on the general quasiperiodic setting, to which the methods we develop here apply.

Finally, Section 6 briefly discusses the extension of our results to some other equations. Considering a simple example, we show that fully nonlinear equations, such as Hamilton-Jacobi type equations, behave very differently from the linear (elliptic) case considered in this work.

We conclude this introduction by mentioning that the present work is the continuation of our long term endeavor $[7,8,9]$ to understand multiscale problems for (realistic if not real) structures that are variants of the idealized structures (periodic, stochastic, etc) traditionally considered for this purpose. Also, we note that most of the results presented here have been announced in [28] and summarized in [11].

\section{Homogenization setting and previous works}

\subsection{Homogenization setting}

General considerations The general perturbation setting we adopt is the following. We consider a scalar-valued coefficient $a$ in (1) of the form

$$
a=a_{0}+b
$$

where $a_{0}$ denotes the (unperturbed) background, and $b$ the perturbation. We will assume throughout the article that all the various $a_{0}$ and $b$ we consider satisfy

$$
\left\{\begin{array}{l}
0<\underline{\mu} \leq a_{0}(x)+b(x), \quad 0<\underline{\mu} \leq a_{0}(x), \quad \text { a.e., for some fixed constant } \underline{\mu}, \\
a_{0} \in L^{\infty}\left(\mathbb{R}^{d}\right), \\
b \in L^{\infty}\left(\mathbb{R}^{d}\right) .
\end{array}\right.
$$

We will also assume that $a_{0}$ is such that the problem

$$
\left\{\begin{array}{l}
-\operatorname{div}\left(a_{0}\left(p+\nabla w_{p, 0}\right)\right)=0 \quad \text { in } \mathbb{R}^{d}, \\
\frac{w_{p, 0}(x)}{1+|x|} \stackrel{|x| \rightarrow \infty}{\longrightarrow} 0,
\end{array}\right.
$$

admits a solution $w_{p, 0}$, unique up to the addition of a constant, that additionally satisfies

$$
\nabla w_{p, 0} \in L^{\infty}\left(\mathbb{R}^{d}\right)
$$


Remark 1 Although we have not worked out all the details of such an extension of our arguments, assuming $\nabla w_{p, 0} \in L_{\text {unif }}^{s}\left(\mathbb{R}^{d}\right)$, for some $s$, instead of (9), should be sufficient for proceeding, up to a modification of our conclusions. One would then typically obtain that, when $b \in L^{r}\left(\mathbb{R}^{d}\right), r \geq 2$, the corrector solution to (5) is such that $\nabla w_{p}-\nabla w_{p, 0}$ belongs to $L^{r^{\prime}}\left(\mathbb{R}^{d}\right)$ for $\frac{1}{r^{\prime}}=\frac{1}{r}+\frac{1}{s}$ in Theorem 4.1.

In many circumstances, we will have to assume local regularity on both the coefficients $a_{0}$ and $b$, namely

$$
a_{0}, b \in C^{0, \alpha} \quad \text { for some } \quad \alpha>0 .
$$

Although this assumption is clearly not standard for the problems considered in homogenization theory, it is necessary for our specific strategy of proof to apply. We do not know whether this assumption is actually necessary for our results to hold. Note that, by classical elliptic regularity theory, the $C^{0, \alpha}$ regularity of $a_{0}$ stated in (10) implies the regularity (9) of the solution to (8).

Remark 2 If (9)-(10) are satisfied, then in fact we have $\nabla w_{p, 0} \in C^{0, \alpha}\left(\mathbb{R}^{d}\right)$ (see Lemma 4.4 below).

Our assumptions In the present contribution, we will make the above general setting more specific in two different ways.

Our first geometric setting is, as briefly mentioned in the introduction, the perturbation of an otherwise perfect periodic setting. More precisely, we assume $a_{0}=a_{p e r}$, that is

$$
a=a_{p e r}+b
$$

where $a_{p e r}$ is periodic and $b$ is a localized perturbation, so that (7) is satisfied. As is well known from classical homogenization theory, (8) then satisfies both the well-posedness property and the additional regularity (9) we require, the latter when we assume (10). In order to express the locality of the perturbation, the natural assumption would be $b(x) \stackrel{|x| \rightarrow \infty}{\longrightarrow} 0$. However, we are unable to address the problem in this full generality and therefore assume that $b$ "vanishes at infinity" in the following weak way:

$$
b \in L^{r}\left(\mathbb{R}^{d}\right), \quad \text { for some } 1 \leq r<+\infty .
$$

Our strategy of proof, and the results we obtain, will critically depend upon the exponent $r$ (see Sections 3 and 4 below).

Our second geometric setting concerns the situation of two different periodic structures separated by a flat interface (see Section 5 below). Up to a rotation, we may always assume that this interface is the hyperplane $\left\{x_{1}=0\right\}$ in $\mathbb{R}^{d}$. In each of the half-spaces $\left\{x_{1}<0, x_{2}, \ldots, x_{d}\right\}$ and $\left\{x_{1}>0, x_{2}, \ldots, x_{d}\right\}$, we have a periodic geometry. Put differently, we assume that $a_{0}$ is of the form

$$
a_{0}(x)=a_{p e r,-,+}(x)=\left\{\begin{array}{lll}
a_{-}(x) & \text { when } x_{1} \leq 0, \\
a_{+}(x) & \text { when } \quad x_{1}>0
\end{array}\right.
$$

where each $a_{ \pm}$denotes a periodic structure. Even when $b=0$ in (6), there is already an interesting situation to consider at the vicinity of the interface, since, in some vague sense at least, this interface is a defect of the periodic structure on each side. The superposition of a nontrivial defect $b$ makes the problem even more interesting.

The difficulty of the problem is essentially related to the commensurability of the two periodic cells, on each side of the interface. Assuming that the periodic cell for $x_{1}<0$ is of the form $Q_{-}=\left[0, R_{1}\right] \times\left[0, R_{2}\right] \times$ $\cdots \times\left[0, R_{d}\right]$ while that for $x_{1}>0$ reads $Q_{+}=\left[0, S_{1}\right] \times\left[0, S_{2}\right] \times \cdots \times\left[0, S_{d}\right]$ with $S_{1}=R_{1}$ and $S_{k} / R_{k} \in \mathbb{Q}$ for all $2 \leq k \leq d$, the same remark holds: one can reduce the problem to a common periodic cell.

The first nontrivial situation is when $S_{1} \neq R_{1}$ and $S_{k} / R_{k} \in \mathbb{Q}$ for all $2 \leq k \leq d$. Up to choosing a common multiple in the directions $k \geq 2$, the background is thus of a common period in those directions, while it has two incommensurable periods in the direction $k=1$. This is the situation we shall study in Section 5.2 below. 
A more intricate situation is that when the periods are incommensurable in a second direction, say $S_{2} / R_{2} \notin \mathbb{Q}$. Then, a quasi periodic structure appears along the interface and the techniques developed in Section 5 are ineffective. We address that case in Section 5.3. We of course note that the two different structures may also be assembled along an inclined interface. The arguments developed here actually allow to also cover such situations.

Remark 3 For simplicity, we use here a periodic background. Similar questions could be considered for a reference background that has a different structure, such as, e.g., a stationary background (see the related Remark 16). The proofs contained in our present study would then have to be adapted, and some of our results might require a certain amount of technicalities, or additional assumptions, to carry over to these more general backgrounds. In the same vein, one could imagine that the background is kept periodic, but the local perturbation we apply is taken stochastic. Many other variants are possible.

Basics of homogenization theory, in the periodic setting We now recall some classical elements of periodic homogenization theory. We refer to the textbooks $[5,22]$ for more details. Consider, for $p=e_{i}$, $i=1,2, \ldots d$, the periodic solution $w_{p, p e r}$ to the periodic corrector equation (2) for the problem where our perturbative term $b$ is omitted. The solution to (2) is unique (up to an irrelevant additive constant). The first order approximation for the solution $u^{\varepsilon}$ to (1) is then defined as

$$
u_{\text {per }}^{\varepsilon, 1}(x)=u^{*}(x)+\varepsilon \sum_{i=1}^{d} \partial_{x_{i}} u^{*}(x) w_{e_{i}, p e r}(x / \varepsilon),
$$

where $e_{i}$ are the canonical vectors of $\mathbb{R}^{d}$ and where $u^{*}$ is the solution to (4) with homogeneous Dirichlet boundary conditions on $\partial \Omega$ and where the entries of the matrix of homogenized coefficients read, for all $1 \leq i, j \leq d$,

$$
\left[A^{*}\right]_{i j}=\text { weak } \lim _{\varepsilon \longrightarrow 0} a_{p e r}(. / \varepsilon)\left(\delta_{i j}+\partial_{j} w_{e_{i}, p e r}(. / \varepsilon)\right)=\int_{Q} a_{p e r}(y)\left(\delta_{i j}+\partial_{j} w_{e_{i}, p e r}(y)\right) d y .
$$

The fact that $u_{\text {per }}^{\varepsilon, 1}$ is an approximation in $H^{1}$ of $u^{\varepsilon}$ (away from the boundary $\partial \Omega$, since peculiar, but well documented effects occur at the vicinity of $\partial \Omega$ ) is a standard fact, for the details of which we refer to the bibliography, see e.g. [5].

\subsection{The $L^{2}$ case}

In [10], we have considered the case (11)-(12), in the specific case $r=2$, and with the regularity assumption $a_{0}=a_{\text {per }} \in C^{0, \alpha}$ which in turn implies the regularity (9). Notice that the regularity of $b$ stated in (10) is not needed in that case $r=2$. Notice also that, although we restricted our presentation in [10] (and its summary here) to the case $a_{0}=a_{\text {per }}$, the periodicity of $a_{0}$ is actually not needed, provided we assume (6)-(7)-(8)-(9). The latter observation is recalled in Remark 4 below.

Well-posedness of the corrector equation We have proved in [10] the following result:

Lemma 2.1 [Lemma 1 in [10]] For all $p \in \mathbb{R}^{d}$, equation (5.1) admits a solution $w_{p}$ such that $\nabla w_{p} \in$ $L_{\text {per }}^{2}+L^{2}\left(\mathbb{R}^{d}\right)$ (and consequently $\lim _{R \rightarrow+\infty} \frac{1}{\left|B_{R}\right|} \int_{B_{R}} \nabla w_{p}=0$, where $B_{R}$ denotes the ball of radius $R$ centered at the origin). Such a solution is unique up to an additive constant.

The proof (see the details in [10]) is based upon the following arguments. We look for $w_{p}$ solution to (5.1) under the form $w_{p}=w_{p, p e r}+\tilde{w}_{p}$ where $w_{p, p e r}$ is the unique (up to an additive constant) periodic solution to (2). The function $\tilde{w}_{p}$ is then solution to

$$
-\operatorname{div}\left(\left(a_{p e r}+b\right)(y) \nabla \tilde{w}_{p}(y)\right)=\operatorname{div}\left(b(y)\left(p+\nabla w_{p, p e r}(y)\right)\right),
$$


and the proof amounts to establishing that such a solution exists, uniquely up to a constant, with $\nabla \tilde{w}_{p} \in$ $L^{2}\left(\mathbb{R}^{d}\right)$. The existence proof classically proceeds by regularization of (14), introducing a small positive scalar $\eta$ and the equation

$$
-\operatorname{div}\left(\left(a_{p e r}+b\right)(y) \nabla \tilde{w}_{p}^{\eta}(y)\right)+\eta \tilde{w}_{p}^{\eta}(y)=\operatorname{div}\left(b(y)\left(p+\nabla w_{p, p e r}(y)\right)\right)
$$

which evidently admits a unique solution by application of the Lax-Milgram Theorem. Note that, at this point, we need $b\left(p+\nabla w_{p, p e r}\right) \in L^{2}\left(\mathbb{R}^{d}\right)$, which is where the regularity assumptions (9)-(10) come in.

A priori estimates are next established, and next one passes to the limit as $\eta$ vanishes. Uniqueness is showing that the only solution $v_{p}=v_{p, p e r}+\tilde{v}_{p}$ with $v_{p, p e r}$ periodic and $\nabla \tilde{v}_{p} \in L^{2}\left(\mathbb{R}^{d}\right)$ to the equation

$$
-\operatorname{div}\left(\left(a_{p e r}+b\right) \nabla v_{p}\right)=0,
$$

is constant. This is achieved upon i) multiplying that equation by $v_{p}$ times a cut-off function $\chi_{R}=\chi(. / R)$, where $\chi$ is a smooth non-negative bounded cut-off function that has value 1 on the ball $B_{1}$ and vanishes outside $B_{2}$, and ii) inferring from this that $\nabla v_{p, p e r}=0$ and next $\nabla \tilde{v}_{p}=0$.

Using Lemma 2.1, it is now straightforward to prove

Lemma 2.2 For all $p \in \mathbb{R}^{d}$, equation (5) admits a solution $w_{p}$, unique up to an additive constant. This solution is indeed such that $\nabla w_{p} \in L_{\text {per }}^{2}+L^{2}\left(\mathbb{R}^{d}\right)$.

The proof is easy and will actually be useful later on in this article. It is an immediate consequence of the following lemma, in the particular case $r=2$.

Lemma 2.3 Assume that $u \in L_{\text {loc }}^{1}\left(\mathbb{R}^{d}\right)$ and $\nabla u \in L^{r}\left(\mathbb{R}^{d}\right)$ for some $1 \leq r<+\infty$. Then $u$ is strictly sub-linear at infinity, in the sense made precise in (5.2).

Proof of Lemma 2.3: For $r>d$, the result is actually a consequence of the Sobolev embeddings, since then $W^{1, r}$ is continuously embedded in some $C^{0, \alpha}$ and the strict sub-linearity follows. For any $1 \leq r<+\infty$, we may argue as follows. We temporarily fix $x \in \mathbb{R}^{d}$, and for $\varepsilon>0$ consider the rescaled function $u_{\varepsilon}(x)=$ $\varepsilon^{\beta} u(x / \varepsilon)$. We compute that $\left\|\nabla u_{\varepsilon}\right\|_{L^{r}\left(\mathbb{R}^{d}\right)}=\varepsilon^{\beta-1+d / r}\|\nabla u\|_{L^{r}\left(\mathbb{R}^{d}\right)}$. Choosing $1>\beta>1-d / r$, which is always possible, we obtain that $\nabla u_{\varepsilon}$ vanishes in $L^{r}\left(\mathbb{R}^{d}\right)$, thus that $u_{\varepsilon}$ converges to a constant in $L^{r^{*}}\left(\mathbb{R}^{d}\right)$, which is exactly saying that $u(x / \varepsilon)=O\left(\varepsilon^{-\beta}\right)$, and thus, since $\beta<1$, that $u$ is strictly sub-linear at infinity. $\diamond$

Remark 4 Actually, in the light of our set of assumptions (7)-(8)-(9), it is worth noticing that the proof performed for $b \in L^{2}$ in [10] and complemented here, does not make explicit use of the periodicity of the unperturbed coefficient $a_{\text {per. }}$. It only uses (7)-(8)-(9). We will return to such considerations in Section 3, when studying the case $b \in L^{r}\left(\mathbb{R}^{d}\right)$ with $r<d$.

Approximation property Using the corrector the existence of which is established in Lemma 2.2, we now construct the non-periodic approximation

$$
u^{\varepsilon, 1}(x)=u^{*}(x)+\varepsilon \sum_{i=1}^{d} \partial_{x_{i}} u^{*}(x) w_{i}(x / \varepsilon),
$$

analogous to (13) and which we hope to be more accurate at the fine scale for the approximation to $u^{\varepsilon}$ solution to (1). We have denoted by $w_{i}=w_{e_{i}}$. We note that

$$
\partial_{j} u^{\varepsilon, 1}=\sum_{i=1}^{d}\left(\delta_{i j}+\partial_{j} w_{i}(. / \varepsilon)\right) \partial_{i} u^{*}+\varepsilon \sum_{i=1}^{d} w_{i}(. / \varepsilon) \partial_{i j}^{2} u^{*},
$$

for all $1 \leq j \leq d$, a fact that we more concisely denote by $\nabla u^{\varepsilon, 1}=(I d+\nabla w(. / \varepsilon)) \nabla u^{*}+\varepsilon w(. / \varepsilon) \nabla \nabla u^{*}$ in the sequel. To assess the quality of the approximation, we perform the following calculation which we 
borrow from [22, p26-27]. For brevity, our calculation is formal. Otherwise stated, we assume the data are sufficiently regular so that all functions manipulated are also regular and all differential equations hold in the strong sense. Our argument may be given a rigorous meaning using a weak formulation for the equations and the actual regularity of the functions involved. We denote by $a_{\varepsilon}()=.\left(a_{p e r}+b\right)(. / \varepsilon)$. We write

$$
-\operatorname{div}\left(a_{\varepsilon} \nabla\left(u^{\varepsilon}-u^{\varepsilon, 1}\right)\right)=-\operatorname{div}\left(\left(A^{*}-a_{\varepsilon}(I d+\nabla w(. / \varepsilon)) \nabla u^{*}(x)\right)\right)+\varepsilon \operatorname{div}\left(a(. / \varepsilon) w(. / \varepsilon) \nabla \nabla u^{*}\right)
$$

since $-\operatorname{div}\left(a_{\varepsilon} \nabla u^{\varepsilon}\right)=-\operatorname{div}\left(A^{*} \nabla u^{*}\right)=f$. We next introduce the matrix valued function

$$
G=A^{*}-a(I d+\nabla w),
$$

which, component by component, reads $[G(x)]_{i j}=\left[A^{*}\right]_{i j}-a(x)\left(\delta_{i j}+\partial_{i} w_{j}(x)\right)$. Because of the corrector equation (5), we know that, component by component, $G$ is divergence-free so there exists a matrix-valued function $B$ such that $G=\operatorname{curl} B$, thus $G(. / \varepsilon)=\varepsilon \operatorname{curl}(B(. / \varepsilon))$. The first term of the right-hand side of (16) may be written

$$
-\operatorname{div}\left(\left(A^{*}-a_{\varepsilon}(I d+\nabla w(. / \varepsilon)) \nabla u^{*}(x)\right)\right)=\varepsilon \operatorname{div}\left(B(x / \varepsilon) \times \nabla \nabla u^{*}\right) .
$$

Inserting this information in (16), we obtain

$$
-\operatorname{div}\left(a_{\varepsilon} \nabla\left(u^{\varepsilon}-u^{\varepsilon, 1}\right)\right)=\varepsilon \operatorname{div}\left(B(. / \varepsilon) \times \nabla \nabla u^{*}\right)+\varepsilon \operatorname{div}\left(a(. / \varepsilon) w(. / \varepsilon) \nabla \nabla u^{*}\right)
$$

The calculation we have just performed is valid both in the periodic case, that is, $a=a_{p e r}$ and $w=w_{p e r}$ is the periodic corrector solution to (2), and in the perturbed case $a=a_{p e r}+b$ with the non-periodic corrector $w$ solution to (5). Our reference calculation is the couple $\left(a=a_{p e r}, w=w_{p e r}\right)$. In that case, the function $B$, potential of the divergence free field $G$, may be taken periodic since $G$ is periodic itself and has zero average. The functions $a, B, w$ appearing in the right-hand side of (17) are uniformly bounded and, since $u^{*}$ is $H^{2}(\Omega)$ (recall that $u^{*}$ solves (4) and that we have taken for simplicity $f \in L^{2}(\Omega)$ ), it is then easy to see that this right-hand side is thus the divergence of a function that is $O(\varepsilon)$ in $L^{2}$ norm. This is the essence of the classical proof of correction in periodic homogenization. In addition, we remark, and this relates to the focus of this article, that the same conclusion of strong convergence holds at the small scale $\varepsilon x$, as easily seen on (17).

For the perturbed case with coefficient $a=a_{p e r}+b$, we argue similarly. The only difference is that, in (17), we need to replace $B$ by $B_{\text {per }}+\tilde{B}$, and $w$ by $w_{\text {per }}+\tilde{w}$, where, according to the properties of $b$,

$$
\operatorname{curl} \tilde{B} \in L^{2} \quad \text { and } \quad \nabla \tilde{w} \in L^{2} .
$$

We need to prove that $\varepsilon \tilde{B}(x / \varepsilon) \rightarrow 0$ as $\varepsilon \rightarrow 0$, and similarly for $\tilde{w}$. Both terms may be treated in the same way.

For the sake of comparison, we finally consider the case where we use the periodic corrector $w_{p e r}$ in the perturbed case. Then the above calculation which led to (17) is now modified. We introduce $\tilde{w}=w-w_{\text {per }}$ and write

$$
\begin{array}{r}
-\operatorname{div}\left(a_{\varepsilon} \nabla\left(u^{\varepsilon}-u_{p e r}^{\varepsilon, 1}\right)\right)=-\operatorname{div}\left(a_{\varepsilon} \nabla\left(u^{\varepsilon}-u^{\varepsilon, 1}\right)\right)-\operatorname{div}\left(a(. / \varepsilon)(\nabla \tilde{w})(. / \varepsilon) \nabla u^{*}(x)\right) \\
-\varepsilon \operatorname{div}\left(a(. / \varepsilon) \tilde{w}(. / \varepsilon) \nabla \nabla u^{*}\right) .
\end{array}
$$

In the right-hand side, the first term is evaluated as in the previous equality (17), and the third term is $O(\varepsilon)$. The difficulty now arises from the presence of the second term which, although strongly convergent (in $H^{-1}$ ) at scale one, does not vanish in any norm at scale $\epsilon x$. A poor quality of the approximation follows.

This leads to the result.

Lemma 2.4 [Lemma 2 from [10]] The solution $u^{\varepsilon}$ to (1) is well approximated in $H^{1}$ norm, both at scale one and at scale $\varepsilon$, by the first order expansion $u^{\varepsilon, 1}(x)=u^{*}(x)+\varepsilon \sum_{i=1}^{d} \partial_{x_{i}} u^{*}(x) w_{e_{i}}(x / \varepsilon)$, in the following sense:

$$
\left\{\begin{array}{l}
\left\|\nabla\left(u^{\varepsilon}-u^{\varepsilon, 1}\right)\right\|_{L^{2}(\Omega)} \longrightarrow 0, \\
\left\|\nabla\left(u^{\varepsilon}-u^{\varepsilon, 1}\right)(\varepsilon .)\right\|_{L^{2}\left(B_{R}\right)} \longrightarrow 0,
\end{array}\right.
$$


where $B_{R}$ of course denotes any arbitrary fixed ball of radius $R$ such that $\varepsilon B_{R} \subset \Omega$. The latter approximation property does not hold in general for the periodic first-order approximation $u_{p e r}^{\varepsilon, 1}(x)=u^{*}(x)+$ $\varepsilon \sum_{i=1}^{d} \partial_{x_{i}} u^{*}(x) w_{e_{i}, p e r}(x / \varepsilon)$ constructed using the periodic corrector $w_{p, p e r}$ solution to (2).

Remark 5 It is actually rather easy to realize that the quality of approximation at the microscopic scale (that is, that of $u^{\varepsilon}(\varepsilon x)$ ) is related to the quality of approximation at the macroscopic scale (that is, that of $u^{\varepsilon}(x)$ ) in a stronger Sobolev norm. Put differently, accounting for the defect modeled by the presence of $b$ by using the corrector $w_{p}$ solution to (5), instead of the corrector $w_{p, 0}$ solution to (8), also allows to quantitatively improve the approximation of $u^{\varepsilon}(x)$ in the $H^{2}$ norm. A result analogous to that of Lemma 2.4 could be stated.

\section{Local defect in $L^{r}, 1 \leq r<d$}

We consider the problem (5) under the assumptions (6)-(7)-(8)-(9).

We assume in addition

$$
b \in L^{r}\left(\mathbb{R}^{d}\right), \quad \text { for some } r<d .
$$

As in Section 2.2, restricted to the case $r=2$, we begin by introducing $\tilde{w}_{p}=w_{p}-w_{p, 0}$ where $w_{p, 0}$ is the solution to (8). The function $\tilde{w}_{p}$ is then sought as the solution to

$$
-\operatorname{div}\left(a \nabla \tilde{w}_{p}\right)=\operatorname{div}\left(b\left(p+\nabla w_{p, 0}\right)\right),
$$

an equation similar to (14). We readily note that, like in the previous section and in sharp contrast with the next section, we will not use, throughout this section, the fact that $a_{0}$ has a particular geometric structure (such as e.g. periodicity). We shall only use the properties (7)-(8)-(9) listed above. See the related Remark 4.

Another comment is in order. The restriction $r<d$ which we assume here on the exponent in (18) is not only related to our technique of proof: $r=d$ is a critical exponent for the question we are investigating. Indeed, consider the simplest possible case of an equation of the form (19), namely, forcefully putting $a=1$ and $w_{p, 0}=0$,

$$
-\Delta \tilde{w}_{p}=\operatorname{div}(b p)
$$

It follows that, as $|x| \rightarrow+\infty$,

$$
\tilde{w}_{p} \propto \int \frac{x-y}{|x-y|^{d}} b(y) d y
$$

which shows that the critical behavior of $b$ is $b(y) \propto|y|^{-1}$, which in turn corresponds to the criticality of the space $L^{d}$. When $b(y) \propto|y|^{-\alpha}$ with $\alpha>1$, (20) vanishes when $|x| \rightarrow \infty$. It suggests, and this will indeed be the case, that, for $r<d, \tilde{w}_{p}$ solution to (19) vanishes at infinity. In contrast, for $r>d$, the solution might even be unbounded, as again suggested by the particular expression (20).

Our purpose is now to establish the following.

Theorem 3.1 Consider the problem (5) under the assumptions (6)-(7)-(8)-(9). Assume (18). Then (5) admits a solution $w_{p}$ which reads $w_{p}=w_{p, 0}+\tilde{w}_{p}$, where $w_{p, 0}$ is the solution to (8), and $\tilde{w}_{p} \in L^{\infty}\left(\mathbb{R}^{d}\right)$ vanishes at infinity. Such a solution is unique up to the addition of a constant.

Remark 6 It is important to note that we are not able to prove that problem (5) has a unique solution. We are only able to prove that $w_{p}$ is unique among the solutions to (5) of the form $w_{p}=w_{p, 0}+\tilde{w}_{p}$, with $\tilde{w}_{p}$ bounded. This is a simple application of Liouville's Theorem. Note, however, that sub-linearity at infinity is not sufficient for this theorem to directly apply to $w_{p}$ and prove its uniqueness. See for instance the counter-examples in [30, $p$ 204] and [33, $p$ 400].

Remark 7 We emphasize that Theorem 3.1 does not make use of the periodicity, or any similar geometric property of the unperturbed coefficient $a_{0}$. It does not use the regularity assumption (10). It is only based upon the properties of the unperturbed corrector problem mentioned in the statement of Theorem 3.1. 
Remark 8 We also note that Theorem 3.1 establishes the strict sub-linearity condition (5.2) but does not show that $\nabla \tilde{w}_{p} \in L^{r}$. Intuitively, this property could be expected to hold because it is true for the Laplacian operator (that is $a \equiv 1$ ) and because $b\left(p+\nabla w_{p, 0}\right) \in L^{r}$ in the right hand side of (19). We will see in the next section some argument that proves the latter property for $r \geq 2$, under additional assumptions on the coefficients $a_{0}$ and $b$.

We now prove Theorem 3.1.

Proof of Theorem 3.1: We begin by briefly proving the uniqueness (up to an additive constant) of $\tilde{w}_{p}$ solution vanishing to infinity of (19), thus that of $w_{p}$. It is obtained by application of a Liouville-type Theorem, (see, for instance, [31, Theorem 4 and its corollary] for the scalar, or more generally symmetric case, and [18, Theorem 8.20] in the general case). The coefficient $a$ is bounded and the solution considered $\tilde{w}_{p}$ vanishes at infinity, thus is bounded. Uniqueness of that solution follows.

We now turn to existence, which is the main issue addressed by Theorem 3.1. We first address the case of dimensions $d \geq 3$ and next that of the dimension $d=2$. Note that the dimension $d=1$ is excluded from our setting since we have assumed (18) throughout this section.

Assume $d \geq 3$. Consider the Green function of the operator $-\operatorname{div}(a \nabla$.$) , that is the solution G(x, y)$, vanishing at infinity in a weak sense to

$$
-\operatorname{div}_{x}\left(a \nabla_{x} G(x, y)\right)=\delta(x-y) .
$$

The existence and uniqueness of such a function $G$ is proved in [21, Theorem 1.1] (see also [12]). Moreover, we have, for all $y \in \mathbb{R}^{d}$,

$$
\|G(., y)\|_{L^{d /(d-2), \infty}}+\left\|\nabla_{x} G(., y)\right\|_{L^{d /(d-1), \infty}} \leq C(d),
$$

where $C(d)$ is a constant that depends on the ambient dimension $d$ and on $a$. This result is a direct consequence of the estimates of [21, Theorem 1.1], or of the proof of [12, Theorem 1].

In (21), we have of course denoted by $L^{q, \infty}$ the weak- $L^{q}$ space, also called the Marcinkiewitz space (see [6, Chapter 1], [35, Chapter 1, Section 8], and also [34] for the definition and standard properties of the Macinkiewitz spaces and more generally those of the Lorentz spaces $\left.L^{p, q}\right)$. It is equipped with the quasi-norm

$$
|f|_{L^{q, \infty}}:=\sup _{s>0}\left[s \text { meas }\{x ;|f(x)|>s\}^{1 / q}\right] .
$$

We next remark that (21) is also true when the roles of $x$ and $y$ are exchanged. Using the linearity of equation (19), the uniqueness we have proven above, and the estimates (21), we may write the solution $\tilde{w}_{p}$ using the representation formula

$$
\tilde{w}_{p}(x)=\int \nabla_{y} G(x, y)\left(b\left(p+\nabla w_{p, 0}\right)\right)(y) d y .
$$

For this purpose, it suffices to remark that when $h \in \mathcal{D}\left(\mathbb{R}^{d}\right)$ is a smooth, $\mathbb{R}^{d}$-valued, compactly supported function, the solution $u$ to $-\operatorname{div}(a \nabla u)=\operatorname{div}(h)$ writes

$$
\tilde{w}_{p}(x)=\int G(x, y) \operatorname{div}(h)(y) d y=-\int \nabla_{y} G(x, y) \cdot h(y) d y .
$$

Next, we notice, in view of (21) (with the exchange of $x$ and $y$ ), that $\nabla_{y} G(x, y) \in L^{d /(d-1), \infty}\left(\mathbb{R}^{d}\right)$. The dual space of that space, namely $L^{d, 1}\left(\mathbb{R}^{d}\right)$, is an interpolate space between $L^{r}=L^{r, r}$ and $L^{\infty}$, for $r<d$. Therefore, using the density of $\mathcal{D}\left(\mathbb{R}^{d}\right)$ in $L^{d, 1}$, we may approximate $b\left(p+\nabla w_{p, 0}\right)$ in $L^{d, 1}\left(\mathbb{R}^{d}\right)$ by a sequence of smooth functions $h_{n} \in \mathcal{D}\left(\mathbb{R}^{d}\right)$ and pass to the limit in (23) to obtain (22).

Next, we need to prove that $\tilde{w}_{p}$ vanishes at infinity. This is again completed using the above density argument. Indeed, using the generalization of the Hölder inequality to Lorentz spaces, we have

$$
\left|\tilde{w}_{p}(x)\right| \leq\left\|\nabla_{y} G(x, y)\right\|_{L^{d /(d-1), \infty}}\left\|b\left(p+\nabla w_{p, 0}\right)\right\|_{L^{d, 1}} .
$$


The rightmost term is bounded from above using the interpolation of $L^{d, 1}$ between $L^{r}=L^{r, r}$ and $L^{\infty}$, for $r<d$. This yields a bound on $\left|\tilde{w}_{p}(x)\right|$ for all $x \in \mathbb{R}^{d}$, that is, an $L^{\infty}$ bound on $\tilde{w}_{p}$. The bound (24) actually shows that $\tilde{w}_{p}$ vanishes at infinity. Indeed, returning to the representation formula (23), we notice that, when $h \in \mathcal{D}\left(\mathbb{R}^{d}\right), u$ vanishes at infinity, since (see [21]), $G(x, y) \longrightarrow 0$ when $|x-y| \longrightarrow \infty$. Next, as above, the density of $\mathcal{D}\left(\mathbb{R}^{d}\right)$ in $L^{d, 1}$ allows to approximate $b\left(p+\nabla w_{p, 0}\right)$ by a sequence $h_{n} \in \mathcal{D}\left(\mathbb{R}^{d}\right)$. The inequality (24) shows that the corresponding sequence $u_{n}$, constructed from $h_{n} \in \mathcal{D}\left(\mathbb{R}^{d}\right)$ approximating $b\left(p+\nabla w_{p, 0}\right)$ in $L^{d, 1}$ converges in $L^{\infty}$ to $\tilde{w}_{p}$. Since all the functions $u_{n}$ vanish at infinity, so does $\tilde{w}_{p}$. This concludes the existence part of Theorem 3.1 for $d \geq 3$.

It remains to establish existence in the case $d=2$, where of course (21) makes no sense. For this purpose, we point out that, according to the appendix of [23] (see also [12]), the Green function is still well defined, satisfies

$$
\forall x \in \mathbb{R}^{2}, \quad \forall y \in \mathbb{R}^{2}, \quad|G(x, y)| \leq C(1+|\log (|x-y|)|),
$$

and is unique in this class. Notice that in sharp contrast to the situation in dimensions $d \geq 3, G(x, y)$ does not vanish when $|x-y| \longrightarrow+\infty$. In addition, instead of (21), we have

$$
\left\|\nabla_{x} G(., y)\right\|_{L^{2, \infty}} \leq C .
$$

This proves that formula (22) still holds true. Indeed, if $h \in \mathcal{D}\left(\mathbb{R}^{2}\right)$, then the solution $u$ of $-\operatorname{div}(a \nabla u)=$ $\operatorname{div}(h)$ again satisfies (23). We now use the following estimation, analogous in spirit to (24):

$$
\|u\|_{L^{\infty}\left(\mathbb{R}^{2}\right)} \leq \sup _{x \in \mathbb{R}^{2}}\left\|\nabla_{y} G(x, \cdot)\right\|_{L^{2, \infty}}\|h\|_{L^{2,1}\left(\mathbb{R}^{2}\right)} .
$$

Since $\mathcal{D}\left(\mathbb{R}^{2}\right)$ is dense in $L^{2,1}\left(\mathbb{R}^{2}\right)$, this implies, arguing as above, that (22) is valid. The fact that $\tilde{w}_{p}$ vanishes at infinity is proved likewise by a density argument. First, if $h \in \mathcal{D}\left(\mathbb{R}^{2}\right)$, then $-\operatorname{div}(a \nabla u)=0$ in $B_{R}^{C}$, for some $R>0$. Hence, a simple application of [31, Theorem 5] proves that $u$ has a limit at infinity. Since $u$ is defined up to the addition of a constant, this limit may be assumed to be 0 . Note that [31, Theorem 5] is stated only for symmetric matrices, but its proof is based only on the Harnack inequality, which is true for non-symmetric matrices (see $[18,24]$ ). We conclude here again by a density argument.

Remark 9 We note in passing that $C(d)$ in (21) in fact only depends on the coefficient a through the ellipticity constant of that function, and not the actual $L^{\infty}$ bound or any further specifics of that function. Intuitively, the larger the coefficient a is, the better for inequality (21). We also note that the proof of existence we have performed above in the specific case $d=2$ actually also carries over to dimensions $d \geq 3$.

\section{Local defect in $L^{r}, r<+\infty$}

We again consider the same problem as in the previous section but now do not restrict $r$ as in (18), that is, we only assume

$$
b \in L^{r}\left(\mathbb{R}^{d}\right), \quad \text { for some } 1 \leq r<\infty .
$$

We will establish Theorem 4.1 below, which is similar in spirit to Theorem 3.1 but requires a different strategy of proof and has a slightly different statement. As shown by the case of the Laplacian briefly summarized at the beginning of Section 3, we cannot expect, indeed, the exact same properties on the solution to (5) whether $r<d$ or not.

Theorem 4.1 Assume that $a=a_{0}+b$ satisfies (6)-(7)-(8)-(9)-(10). Assume that $a_{0}=a_{\text {per }}$ is periodic. Then, problem (5) has a solution $w_{p}$ such that $w_{p}=w_{p, 0}+\tilde{w}_{p}$, where $w_{p, 0}$ is the periodic corrector, that is, the solution to (8), and

- if $1 \leq r<d$, then $\nabla \tilde{w}_{p} \in L^{r}, \lim _{|x| \rightarrow+\infty} \tilde{w}_{p}(x)=0$, and the solution $w_{p}$ is unique among those satisfying $w_{p}=v_{\text {per }}+v$, where $v_{\text {per }}$ is periodic and $\nabla v \in L^{r}$;

- if $2 \leq r$, then $\nabla \tilde{w}_{p} \in L^{r}$. In addition, the solution $w_{p}$ is unique in the class of solutions $w_{p}=v_{\mathrm{per}}+v$, where $v_{\text {per }}$ is periodic and $\nabla v \in L^{r}$. 
Remark 10 The first assertion $\lim _{|x| \rightarrow+\infty} \tilde{w}_{p}(x)=0$ of Theorem 4.1 has actually already been established in Theorem 3.1, under less restrictive assumptions on the coefficient since the periodicity and the $C^{0, \alpha}$ regularity of $a_{0}$ were not assumed there. The interest of Theorem 4.1 is that its proof, different from that of Theorem 3.1, also applies to exponents $r \geq d$.

To prepare ourselves for the proof of Theorem 4.1, we first establish the following classical result which generalizes to the operator - $\operatorname{div}(a \nabla$. $)$ the elementary pointwise estimations known on the first and second gradient of the Green function of the Laplace operator. The generalization requires some local integration, and does not hold pointwise.

Lemma 4.2 Assume that the coefficient a satisfies (6)-(7). Then, for all $1 \leq q \leq 2$, there exists a constant $C$ such that, for all $R>0$ and all $x \in \mathbb{R}^{d}$, the Green function $G$, solution to

$$
-\operatorname{div}_{x}\left(a \nabla_{x} G(x, y)\right)=\delta(x-y)
$$

satisfies

$$
\begin{aligned}
& \int_{B_{2 R}(x) \backslash B_{R}(x)}\left|\nabla_{y} G(x, y)\right|^{q} d y \leq \frac{C}{R^{d(q-1)-q}}, \\
& \int_{B_{2 R}(x) \backslash B_{R}(x)}\left|\nabla_{x} \nabla_{y} G(x, y)\right|^{q} d y \leq \frac{C}{R^{d(q-1)}},
\end{aligned}
$$

where $B_{2 R}(x) \backslash B_{R}(x)=\{y, R \leq|x-y| \leq 2 R\}$ denotes the annular region enclosed between the balls of radius $R$ and $2 R$.

Remark 11 Note that, according to the results of [21] and [23], the following pointwise estimates for G still hold true: if $d=2$,

$$
\forall x, y \in \mathbb{R}^{2}, \quad|G(x, y)| \leq C(1+|\log | x-y||),
$$

and if $d \geq 3$

$$
\forall x, y \in \mathbb{R}^{d}, \quad 0 \leq G(x, y) \leq \frac{1}{|x-y|^{d-2}} .
$$

In the course of the proof of Lemma 4.2, the following, also classical, result will be useful:

\section{Lemma 4.3 (Caccioppoli inequality, Proposition 2.1 p 76 in [17])}

Assume $a$ is elliptic and bounded, that is, in the simple scalar case, satisfies (7). Assume $-\operatorname{div}(a \nabla u)=0$ in the ball $B_{2 R}$. Then

$$
\int_{B_{R}}|\nabla u|^{2} \leq \frac{C}{R^{2}} \int_{B_{2 R}}|u|^{2}
$$

where the constant $C$ only depends upon a.

Remark 12 Note that no regularity on the coefficient a is needed for Lemma 4.3 to hold true.

For the convenience of the reader and for consistency, we provide below a possible proof of Lemma 4.3.

Proof of Lemma 4.3: We follow the proof of [17, Proposition 2.1, Chapter III]. Let $\eta_{R}$ be a cut-off function satisfying the following:

$$
\eta_{R} \in \mathcal{D}\left(B_{2 R}\right), \quad 0 \leq \eta_{R} \leq 1, \quad \eta_{R \mid B_{R}}=1, \quad\left|\nabla \eta_{R}\right| \leq \frac{c}{R} .
$$

We multiply the equation by $u \eta_{R}^{2}$, use the Green formula and find

$$
0=\int-\operatorname{div}(a \nabla u) u \eta_{R}^{2}=\int(a \nabla u) \cdot \nabla\left(u \eta_{R}\right) \eta_{R}+\int u \eta_{R}(a \nabla u) \cdot \nabla \eta_{R} .
$$


Since $(a \nabla u) \eta_{R}=a \nabla\left(u \eta_{R}\right)-u a \nabla \eta_{R}$, we have

$$
\int(a \nabla u) \eta_{R} \cdot \nabla\left(u \eta_{R}\right)=\int a \nabla\left(u \eta_{R}\right) \cdot \nabla\left(u \eta_{R}\right)-\int a u \nabla \eta_{R} \cdot \nabla\left(u \eta_{R}\right),
$$

and thus, using (31) in the left hand side, $-\int u \eta_{R}(a \nabla u) \cdot \nabla \eta_{R}=\int a \nabla\left(u \eta_{R}\right) \cdot \nabla\left(u \eta_{R}\right)-\int a u \nabla \eta_{R} \cdot \nabla\left(u \eta_{R}\right)$. Therefore,

$$
\int a \nabla\left(u \eta_{R}\right) \cdot \nabla\left(u \eta_{R}\right)=\int u\left(a \nabla \eta_{R}\right) \cdot \nabla\left(u \eta_{R}\right)-\int u a \nabla\left(u \eta_{R}\right) \cdot \nabla \eta_{R}+\int u^{2}\left(a \nabla \eta_{R}\right) \cdot \nabla \eta_{R} .
$$

Using Cauchy-Schwarz inequality, together with the ellipticity of $a$, we obtain

$$
\underline{\mu} \int\left|\nabla\left(u \eta_{R}\right)\right|^{2} \leq 2\|a\|_{L^{\infty}}\left(\int u^{2}\left|\nabla \eta_{R}\right|^{2}\right)^{1 / 2}\left(\int\left|\nabla\left(u \eta_{R}\right)\right|^{2}\right)^{1 / 2}+\|a\|_{L^{\infty}} \int u^{2}\left|\nabla \eta_{R}\right|^{2} .
$$

This clearly implies that

$$
\frac{\underline{\bar{\mu}}}{2} \int\left|\nabla\left(u \eta_{R}\right)\right|^{2} \leq\left(\frac{2\|a\|_{L^{\infty}}^{2}}{\underline{\mu}}+\|a\|_{L^{\infty}}\right) \int u^{2}\left|\nabla \eta_{R}\right|^{2} .
$$

Hence, using (30), we find

$$
\frac{\overline{\bar{\mu}}}{\int_{B_{R}}}|\nabla u|^{2} \leq\left(\frac{2\|a\|_{L^{\infty}}^{2}}{\underline{\mu}}+\|a\|_{L^{\infty}}\right) \frac{c^{2}}{R^{2}} \int_{B_{2 R}} u^{2},
$$

which concludes the proof of Lemma 4.3.

Remark 13 In the above proof, it is clear that the constant $C$ in (29) only depends on $\frac{\|a\|_{L} \infty}{\underline{\mu}}$.

We are now able to proceed with the proof of Lemma 4.2, which we also provide here for the sake of consistency:

Proof of Lemma 4.2: We first prove, for any $q \in[1,2]$,

$$
\int_{B_{2 R} \backslash B_{R}}\left|\nabla_{x} G(x, y)\right|^{q} d y \leq \frac{C}{R^{d(q-1)-q}} .
$$

We perform the proof of (32) first for dimensions $d \geq 3$.

In the case $q=2$, we use Lemma 4.3 , which implies that

$$
\int_{B_{R / 2}\left(x_{0}\right)}\left|\nabla_{x} G(x, y)\right|^{2} d x \leq \frac{C}{R^{2}} \int_{B_{R}\left(x_{0}\right)}|G(x, y)|^{2} d x .
$$

Next, we cover $B_{2 R} \backslash B_{R}=\{x, R<|x-y|<2 R\}$ by balls $B_{R / 2}\left(x_{i}\right)$, for some points $x_{i}$ such that $5 R / 4<$ $\left|x_{i}\right|<7 R / 4$, in such a way that (i) a finite number of such $x_{i}$ is sufficient to cover the ring $B_{2 R} \backslash B_{R}$ and that (ii) any point in $B_{2 R} \backslash B_{R}$ belongs to at most $K$ balls $B_{R / 2}\left(x_{i}\right)$, for some $K$ that is independent of the radius $R$. This is easily seen to be possible .

The above estimate holds for any couple of balls $\left(B_{R / 2}\left(x_{i}\right), B_{R}\left(x_{i}\right)\right)$. We sum all such estimates over the finite number of indices $i$ and obtain

$$
\int_{B_{2 R} \backslash B_{R}}\left|\nabla_{x} G(x, y)\right|^{2} d x \leq \frac{C K}{R^{2}} \int_{B_{11 R / 4} \backslash B_{R / 4}}|G(x, y)|^{2} d x .
$$

Then, since $d \geq 3$, we get, using (28),

$$
\int_{B_{2 R} \backslash B_{R}}\left|\nabla_{x} G(x, y)\right|^{2} d x \leq \frac{C K}{R^{2}} \int_{R / 4}^{11 R / 4} \frac{r^{d-1}}{r^{2 d-4}} d r \leq \frac{C}{R^{d-2}} .
$$


This proves the case $q=2$. For $q<2$, we simply apply the Hölder inequality and use (34):

$$
\begin{aligned}
\int_{B_{2 R} \backslash B_{R}}\left|\nabla_{x} G(x, y)\right|^{q} d x & \leq\left(\int_{B_{2 R} \backslash B_{R}}\left|\nabla_{x} G(x, y)\right|^{2} d x\right)^{q / 2} R^{d(1-q / 2)} \\
& \leq C R^{-(d-2) q / 2+d-d q / 2}=C R^{-d q+d+q} .
\end{aligned}
$$

We thus have proved (32) for $d \geq 3$.

We next prove (32) for $d=2$. For this purpose, we use the following inequality, valid for any $\beta \in(0,2]$, and which expresses and quantifies the continuous embedding of $L^{2, \infty}$ into $L^{r}$ for $r<2$ on bounded domains:

$$
\forall f \in L^{2, \infty}(\Omega), \quad \int_{\Omega}|f|^{2-\beta} \leq C_{\beta}|\Omega|^{\beta / 2}\|f\|_{L^{2, \infty}(\Omega)}^{2-\beta},
$$

where $C_{\beta}=4 \frac{1+2^{-\beta}}{\left(2^{\beta}-1\right)^{(2-\beta) / 2}}$ is suitable. This estimate is proved for instance in the Appendix of [12]. We apply it to $f=\nabla_{x} G$ and $\Omega=B_{2 R} \backslash B_{R}$, and find, using (25)

$$
\int_{B_{2 R} \backslash B_{R}}\left|\nabla_{x} G\right|^{2-\beta} \leq C_{\beta} C R^{\beta} .
$$

This implies (32) for $q=2-\beta \in[0,2)$. Finally, in order to prove (32) for $q=2$, we fix $y$ and first point out that, integrating the equation $-\operatorname{div}_{x}\left(a(x) \nabla_{x} G(x, y)\right)=\delta_{y}(x)$ on the set $\{x, G(x, y) \geq s\}$ (which contains $y$ ) for some $s \in \mathbb{R}$, that

$$
1=\int_{G \geq s}-\operatorname{div}_{x}\left(a \nabla_{x} G\right) d x=-\int_{G=s}\left(a \nabla_{x} G\right) \cdot n_{s}
$$

where $n_{s}$ denotes the outward normal to the set $\{x, G(x, y) \geq s\}$. Note that, here, we have implicitly assumed that the set $\{x, G(x, y) \geq s\}$ is Lipschitz-continuous, so that its outer normal is well defined and we can integrate by parts. This may not be the case, given the regularity of $G$. However, using the co-aera formula (see [2, Theorem 3.40]), it is simple to prove that, since $G \in C^{0, \alpha}$ away from $x=y$, that this set is Lipschitz-continuous for almost all $s \in \mathbb{R}$. This is sufficient for our purpose here.

Next, we multiply the equation by $G$ and integrate on $\{m \leq G \leq M\}$ for some $m \leq M$. This gives

$0=\int_{M \geq G \geq m}-\operatorname{div}_{x}\left(a \nabla_{x} G\right) G d x=\int_{G=M} G\left(a \nabla_{x} G\right) \cdot n_{M}-\int_{G=m} G\left(a \nabla_{x} G\right) \cdot n_{m}+\int_{M \geq G \geq m}\left(a \nabla_{x} G\right) \cdot \nabla_{x} G$.

Hence, using (36), we have

$$
\int_{M \geq G \geq m}\left(a \nabla_{x} G\right) \cdot \nabla_{x} G=M-m .
$$

Next, we define, for $R>0, m_{R}=\inf \left\{G(x, y), x \in B_{2 R} \backslash B_{R}\right\}$, and $M_{R}=\sup \left\{G(x, y), x \in B_{2 R} \backslash B_{R}\right\}$. We have

$$
B_{2 R} \backslash B_{R} \subset\left\{m_{R} \leq G \leq M_{R}\right\} .
$$

Hence,

$$
\int_{B_{2 R} \backslash B_{R}}\left|\nabla_{x} G\right|^{2} d x \leq C \int_{m_{R} \leq G \leq M_{R}}\left(a \nabla_{x} G\right) \cdot \nabla_{x} G=M_{R}-m_{R} .
$$

We apply the estimate (30) of [14], namely here

$$
\|G\|_{C^{0, \alpha}\left(B_{r}\right)} \leq C r^{-\alpha}\|\nabla G\|_{L^{2, \infty}\left(B_{2 r}\right)},
$$

for all $r$ such that $B_{2 r} \subset B_{2 R} \backslash B_{R}$. In view of (25), that estimate implies that $M_{R}-m_{R}$ is bounded independently of $R$. This proves (32) in the case $q=2$.

At this stage, we have proved (32). We next point out that, in all generality and for non necessarily symmetric matrix-valued coefficients $a, H(x, y)=G(y, x)$ is the Green function of the operator $-\operatorname{div}\left(a^{T} \nabla \cdot\right)$, 
where $a^{T}$ is the transpose matrix of $a$, which satisfies the same hypotheses as $a$. Hence, we may apply (32) to $H$, finding (26).

Finally, we use the fact that $\nabla_{y} G$ satisfies $\operatorname{div}_{x}\left(a \nabla_{x} \nabla_{y} G\right)=0$ in $B_{2 R} \backslash B_{R}$. Hence, we use the Caccioppoli inequality (29) once again, finding (33), namely

$$
\int_{B_{2 R} \backslash B_{R}}\left|\nabla_{x} \nabla_{y} G(x, y)\right|^{2} d x \leq \frac{C}{R^{2}} \int_{B_{2 R} \backslash B_{R}}\left|\nabla_{y} G(x, y)\right|^{2} d x .
$$

Hence, applying (26), we find

$$
\int_{B_{2 R} \backslash B_{R}}\left|\nabla_{x} \nabla_{y} G(x, y)\right|^{2} d x \leq \frac{C}{R^{d}},
$$

that is, (27) for $q=2$. Here again, we conclude for $q \in[1,2]$ using the Hölder inequality, exactly as in (35). This concludes the proof of Lemma 4.2.

Before we are in position to prove Theorem 4.1, we need yet another technical lemma:

Lemma 4.4 Assume that (9) and (10) are satisfied, together with (7). Then $\nabla w_{p, 0} \in C^{0, \alpha}\left(\mathbb{R}^{d}\right)$.

Proof of Lemma 4.4: We simply note that

$$
-\operatorname{div}\left(a \nabla\left(p . x+w_{p, 0}\right)\right)=0,
$$

thus, applying Theorem 3.2 (page 88) of [17], we have

$$
\left\|\nabla\left(p . x+w_{p, 0}\right)\right\|_{C^{0, \alpha}\left(B_{1}\right)} \leq C\left\|\nabla\left(p . x+w_{p, 0}\right)\right\|_{L^{2}\left(B_{2}\right)},
$$

on all couple of balls sharing the same center. It follows that $\nabla w_{p, 0} \in C^{0, \alpha}\left(\mathbb{R}^{d}\right)$ since, by assumption, $\nabla w_{p, 0} \in L^{\infty}\left(\mathbb{R}^{d}\right)$.

We now finally turn to the

Proof of Theorem 4.1: We consider $a_{0}=a_{\text {per }}$ periodic (this is essential in our argument) satisfying the additional regularity (10), and $b \in L^{r}\left(\mathbb{R}^{d}\right), r \geq 2$.

We begin by proving uniqueness. To this end, we assume that we have two solutions and denote by $v$ their difference, which reads

$$
v=u_{\text {per }}+u, \quad u_{\text {per periodic }, ~}^{\nabla u \in L^{r}} \text {. }
$$

One can shift the problem to infinity in such a way that both $\nabla u$ and $b$ vanish. We obtain $\operatorname{div}\left(a_{\text {per }} \nabla u_{\text {per }}\right)=0$. Hence, $\nabla u_{\text {per }}=0$. Thus, we have

$$
-\operatorname{div}\left(a_{p e r} \nabla u\right)=\operatorname{div}(b \nabla u) .
$$

Now, $b \nabla u \in L^{r / 2}\left(\mathbb{R}^{d}\right)$, since $b \in L^{r}$ and $\nabla u \in L^{r}$. Hence, applying the results of [3], $\nabla u \in L^{r / 2}$. This implies that $b \nabla u \in L^{r / 3}$, hence that $\nabla u \in L^{r / 3}$. Repeating this argument, we find that $\nabla u \in L^{2}$. Since $-\operatorname{div}(a \nabla u)=0$, the results of [10] imply that $u$ is a constant.

The rest of the proof is devoted to existence. We represent a solution $\tilde{w}_{p}$ to (19) by

$$
\tilde{w}_{p}(y)=\int \nabla_{x} G(y, x)\left[b\left(p+\nabla w_{p, p e r}(x)\right)\right] d x .
$$

Using an approximation argument like for (15), it is easy to prove that such a solution exists. We now prove that this solution is such that $\nabla \tilde{w}_{p} \in L^{r}\left(\mathbb{R}^{d}\right)$. Lemma 2.3 will then show the strict sub-linearity (19).

For this purpose, we fix $x_{0} \in \mathbb{R}^{d}$, and define a cut-off function $\chi$ around it:

$$
\chi \in \mathcal{D}\left(\mathbb{R}^{d}\right), \quad \chi_{\mid B_{1}\left(x_{0}\right)}=1, \quad \chi_{\mid B_{2}^{C}\left(x_{0}\right)}=0, \quad \chi \geq 0, \quad|\nabla \chi| \leq 2 .
$$


We split (37) into different terms:

$$
\tilde{w}_{p}(y)=\underbrace{\int_{\mathbb{R}^{d}} \nabla_{x} G(y, x)\left[b\left(p+\nabla w_{p, p e r}(x)\right)\right] \chi(x) d x}_{:=w_{1}(y)}+\underbrace{\int_{\mathbb{R}^{d}} \nabla_{x} G(y, x)\left[b\left(p+\nabla w_{p, p e r}(x)\right)\right](1-\chi(x)) d x .}_{:=w_{2}(y)}
$$

We start with the first term:

$$
\left\|w_{1}\right\|_{L^{\infty}\left(B_{1}\left(x_{0}\right)\right)} \leq\left\|b\left(\nabla w_{p, p e r}+p\right)\right\|_{L^{\infty}\left(\mathbb{R}^{d}\right)} \sup _{y \in B_{1}\left(x_{0}\right)}\left(\int_{B_{2}\left(x_{0}\right)}\left|\nabla_{x} G(y, x)\right| d x\right) \leq C\|b\|_{L^{\infty}\left(\mathbb{R}^{d}\right)},
$$

where $C$ does not depend on $x_{0}$ nor on $\tilde{w}_{p}$. Next, we apply Theorem 3.2 (page 88) of [17], which, since $-\operatorname{div}\left(a \nabla w_{1}\right)=\operatorname{div}\left(b\left(p+\nabla w_{p, p e r}\right) \chi\right)$, implies that

$$
\left\|\nabla w_{1}\right\|_{C^{0, \alpha}\left(B_{1 / 2}\left(x_{0}\right)\right)} \leq C\left(\left\|\nabla w_{1}\right\|_{L^{2}\left(B_{1}\left(x_{0}\right)\right)}+\left\|b\left(\nabla w_{p, p e r}+p\right)\right\|_{C^{0, \alpha}\left(\mathbb{R}^{d}\right)}\right) .
$$

Here, we have used that $a \in C^{0, \alpha}$ and that $b \in C^{0, \alpha}$, that is, (10). Moreover, we bound the $L^{2}$ norm of $\nabla w_{1}$ using Caccioppoli's inequality (29), with $R=1$, finding

$$
\left\|\nabla w_{1}\right\|_{C^{0, \alpha}\left(B_{1 / 2}\left(x_{0}\right)\right)} \leq C\left(\left\|w_{1}\right\|_{L^{2}\left(B_{1}\left(x_{0}\right)\right)}+\left\|b\left(\nabla w_{p, p e r}+p\right)\right\|_{C^{0, \alpha}\left(\mathbb{R}^{d}\right)}\right) .
$$

Next, using (38), we have

$$
\left\|\nabla w_{1}\right\|_{L^{\infty}\left(B_{1 / 2}\left(x_{0}\right)\right)} \leq C\|b\|_{C^{0, \alpha}\left(\mathbb{R}^{d}\right)},
$$

where $C$ does not depend on $x_{0}$ nor on $\tilde{w}_{p}$.

Notice that the above argument is actually the only one where we are using the Hölder regularity assumed on $b$ in (10).

We now turn our attention to the second term. We differentiate it, and split the integral into, of course, dyadic rings:

$$
\nabla w_{2}(y)=\sum_{i \in \mathbb{N}} \int_{B_{2^{i+1}}(y) \backslash B_{2^{i}}(y)} \nabla_{x} \nabla_{y} G(y, x)\left[b\left(p+\nabla w_{p, p e r}(x)\right)\right] d x .
$$

We apply (27), for $q$ such that $1 / q+1 / r=1$ (this is where we use our assumption that $r \geq 2$, so that $q \leq 2$ in $(27))$, and get

$$
\left|\nabla w_{2}(y)\right| \leq \sum_{i \in \mathbb{N}}\left\|\nabla_{x} \nabla_{y} G\right\|_{L^{q}\left(B_{2^{i+1}} \backslash B_{2^{i}}\right)}\left\|b\left(p+\nabla w_{p, p e r}\right)\right\|_{L^{r}\left(B_{2^{i+1}} \backslash B_{2^{i}}\right)} \leq \sum_{i \in \mathbb{N}} \frac{C}{2^{i d(1-1 / q)}}\|b\|_{L^{r}\left(\mathbb{R}^{d}\right)} .
$$

This and (39) imply that $\nabla \tilde{w}_{p} \in L^{\infty}\left(B_{1}\left(x_{0}\right)\right)$, with

$$
\left\|\nabla \tilde{w}_{p}\right\|_{L^{\infty}\left(B_{1 / 2}\left(x_{0}\right)\right)} \leq C\left(\|b\|_{L^{r}\left(\mathbb{R}^{d}\right)}+\|b\|_{C^{0, \alpha}\left(\mathbb{R}^{d}\right)}\right),
$$

where $C$ depends only on $a$. Since $x_{0}$ is arbitrary, this proves that $\nabla \tilde{w}_{p} \in L^{\infty}\left(\mathbb{R}^{d}\right)$.

We next write (19) as

$$
-\operatorname{div}\left(a_{p e r} \nabla \tilde{w}_{p}\right)=\operatorname{div}\left(b \nabla \tilde{w}_{p}\right)+\operatorname{div}\left(b\left(p+\nabla w_{p, p e r}\right)\right) .
$$

The classical result of [3], which technically requires the periodicity and the regularity (10) of the coefficient, allows to derive that

$$
\left\|\nabla \tilde{w}_{p}\right\|_{L^{r}} \leq C\|b\|_{L^{r}}\left(\left\|\nabla \tilde{w}_{p}\right\|_{L^{\infty}}+\left\|p+\nabla w_{p, p e r}\right\|_{L^{\infty}}\right) .
$$

This concludes the proof of Theorem 4.1.

Remark 14 For $r \leq 2$, we have $b \in L^{r} \cap L^{\infty}$, hence $b \in L^{2}$. Thus, the results of [10] apply. This proves that $w_{p}$ exists, with $\nabla \tilde{w}_{p} \in L^{2}$. This implies that $\tilde{w}_{p}$ is strictly sublinear at infinity (see Lemma 2.3). However, this does not imply that $\nabla \tilde{w}_{p} \in L^{r}$. 
Remark 15 The questions we address here are of course closely related to the theory of Calderon-Zygmund operators. Using the specific form of the coefficient in the divergence operator (in particular when necessary the periodicity of the coefficient), we establish the integrability of the solution assuming that of the right-hand side. The question is a particular case of the general question: under what assumptions does the linear operator implicitly defined by (19), and that maps $b$ to $\nabla \tilde{w}_{p}$, transform a function in $L^{r}, 1 \leq r<+\infty, L^{\infty}$, or BMO to a function in those spaces?

Remark 16 Our proof makes an essential use of the results of [3], proved under the regularity assumption (10) and the periodicity of the coefficient. The recent reference [19] establishes results on the asymptotic behaviour of the Green function for an operator in divergence form with random stationary coefficients and a zero-order term, but without regularity. It is mentioned in Remark 2.2 of that reference and comments afterwards that the technique of proof carries over to the random case and the periodic case, both without zero-order term. Since estimates on the Green function in turn imply, using the David-Journé theory, that the corresponding operator acts from $L^{p}$ to $L^{p}$, such results would allow one to extend our own results to the case where the regularity assumption (10) is not satisfied, and/or where periodicity is replaced by stationarity.

Remark 17 In the special case of $b \in L^{r} \cap L^{\infty}$ with $\|b\|_{L^{\infty}}$ small, it is possible to give a different and much simpler proof. Indeed, it is possible to write (19) as follows:

$$
-\operatorname{div}\left(a_{0} \nabla \tilde{w}_{p}\right)=\operatorname{div}\left(b \nabla \tilde{w}_{p}\right)+\operatorname{div}\left(b\left(p+\nabla w_{p, p e r}\right)\right),
$$

where $w_{p, p e r}$ is the solution to the periodic corrector problem (that is, with $a_{0}$ ). We then apply a fixed-point strategy, computing the following sequence: $\tilde{w}_{p}^{0}=0$, and

$$
\forall n \in \mathbb{N}, \quad-\operatorname{div}\left(a_{0} \nabla \tilde{w}_{p}^{n+1}\right)=\operatorname{div}\left(b \nabla \tilde{w}_{p}^{n}\right)+\operatorname{div}\left(b\left(p+\nabla w_{p, p e r}\right)\right) .
$$

This defines a sequence $\nabla \tilde{w}_{p}^{n} \in L^{r}$, thanks to the results of [3]. Moreover, the corresponding operator is continuous, so that we have

$$
\left\|\nabla \tilde{w}_{p}^{n+1}-\nabla \tilde{w}_{p}^{n}\right\|_{L^{r}} \leq C\|b\|_{L^{\infty}}\left\|\nabla \tilde{w}_{p}^{n}-\nabla \tilde{w}_{p}^{n-1}\right\|_{L^{r}},
$$

where the constant $C$ depends only on $a_{0}$. Hence, if $\|b\|_{L^{\infty}}<C^{-1}$, the sequence $\nabla \tilde{w}_{p}^{n}$ converges in $L^{r}$, which gives the existence. Uniqueness is dealt with as in the proof of Theorem 4.1.

\section{$5 \quad$ Interfaces between periodic structures}

In this Section, we address a different problem than that studied in Sections 3 and 4. We consider two different periodic structures separated by an hyperplane. In Section 5.2, we consider the simplest case in which this hyperplane is aligned with the periodic cells of both periodic structures considered on each side. We additionally assume that, in the directions parallel to this hyperplane, the two structures share a common periodic structure (see Figure 1 and condition (47)). This situation is the closest possible situation to that of a localized defect. In Section 5.3, we consider the significantly more intricate case when the hyperplane is still parallel to both periodic cells but where the two periodic structures do not share a common periodic cell along the directions of that hyperplane. Up to geometric transformations and possibly a slight adaptation of the arguments of our proofs, this situation is the generic setting for interfaces between two different periodic structures. A quasiperiodic feature emerges in the directions of the hyperplane separating the periodic structures. We will study that situation in details.

\section{$5.1 \quad$ Geometric setting}

We now make precise the geometric setting. We assume that the interface is located at $\left\{x_{1}=0\right\}$, as it is the case in Figure 1. The coefficient $a$ is defined as

$$
a(x)= \begin{cases}a_{-}(x) & \text { if } x_{1}<0 \\ a_{+}(x) & \text { if } x_{1}>0\end{cases}
$$




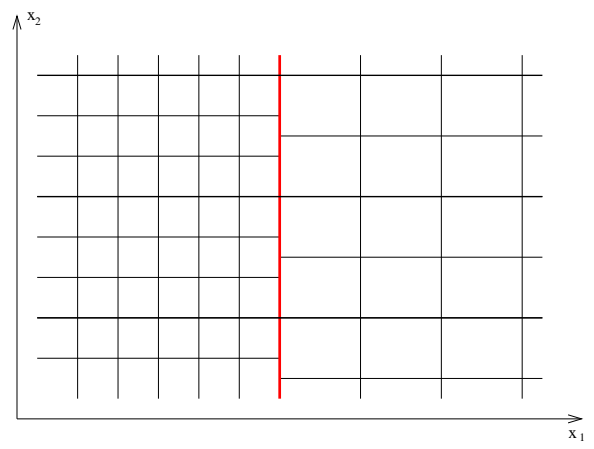

Figure 1: The case of two periodic structures having common periods in the direction of the interface.

with

$$
a_{-} \text {is periodic of period } R_{i} \text { in } x_{i}, \forall i \in\{1, \cdots, d\}
$$

and

$$
a_{+} \text {is periodic of period } S_{i} \text { in } x_{i}, \forall i \in\{1, \cdots, d\} .
$$

As announced above, Section 5.2 below assumes a condition of commensurability (namely (47)) on the periods $R_{i}$ and $S_{i}$ for $i \geq 2$, while Section 5.3 removes that condition.

Applying homogenization theory to this setting, it is easily seen that the homogenized operator corresponding to (1) - (40) -(41) -(42) is

$$
-\operatorname{div}\left(a^{*} \nabla \cdot\right)
$$

where

$$
a^{*}(x)= \begin{cases}a_{-}^{*} & \text { if } x_{1}<0 \\ a_{+}^{*} & \text { if } x_{1}>0\end{cases}
$$

and the homogenized coefficients $a_{ \pm}^{*}$ are the periodic homogenized coefficients associated with $a_{ \pm}$, namely

$$
\forall p \in \mathbb{R}^{d}, \quad a_{ \pm}^{*} p=\left\langle a_{ \pm}\left(\nabla w_{ \pm}+p\right)\right\rangle .
$$

In $(44),\langle\cdot\rangle$ denotes the mean value of periodic functions, and the correctors $w_{ \pm}$are solution to

$$
\left\{\begin{array}{l}
-\operatorname{div}\left(a_{+}\left(\nabla w_{+}+p\right)\right)=0 \\
w_{+} \text {is } S_{i}-\text { periodic in } x_{i}, \forall 1 \leq i \leq d
\end{array}\right.
$$

and

$$
\left\{\begin{array}{l}
-\operatorname{div}\left(a_{-}\left(\nabla w_{-}+p\right)\right)=0 \\
w_{-} \text {is } R_{i}-\text { periodic in } x_{i}, \forall 1 \leq i \leq d
\end{array}\right.
$$

We know that $w_{+}$and $w_{-}$both exist and are both unique up to the addition of a constant.

\subsection{Matching interfaces}

We assume in this Section that

$$
\forall 2 \leq i \leq d, \quad \frac{S_{i}}{R_{i}} \in \mathbb{Q} .
$$

Note that $S_{1} / R_{1}$ can be any positive finite number, but of course if that ratio is also a rational number then the problem is only interesting when $a_{-} \neq a_{+}$(otherwise the problem is periodic throughout the whole space).

For all $2 \leq i \leq d$, we may find a common period $T_{i}$ multiple of both $R_{i}$ and $S_{i}$. Hence, in all the directions except $x_{1}$, the problem is periodic. In contrast, in the direction $x_{1}$, even if $S_{1}=R_{1}$, no periodicity is present, unless $S_{i}=R_{i}$ for all $i$ and $a_{+}=a_{-}$which again is the uninteresting case we exclude. The situation considered here enjoys some formal similarity with that of a local defect previously considered in 
this article in that, in the $x_{1}$ direction, the coefficient $a$ is periodic at infinity, that is, as $x_{1} \rightarrow+\infty$ on the one hand, and as $x_{1} \rightarrow-\infty$ on the other hand. Although these periods may be different, the problem is thus intuitively similar in nature with the case of a point-defect in the $x_{1}$-direction.

We denote by

$$
D=\mathbb{R} \times\left[0, T_{2}\right] \times \cdots \times\left[0, T_{d}\right], \quad D_{ \pm}=\mathbb{R}^{ \pm} \times\left[0, T_{2}\right] \times \cdots \times\left[0, T_{d}\right],
$$

so that $D=D_{+} \cup D_{-}$. We define the corrector problem:

$$
\left\{\begin{array}{l}
-\operatorname{div}\left(a\left(\nabla w_{p}+p\right)\right)=-\operatorname{div}\left(a^{*} p\right) \quad \text { in } \mathbb{R}^{d} \\
w_{p} \text { is } T_{i}-\text { periodic in } x_{i}, \forall 2 \leq i \leq d \\
\nabla\left(w_{p}-w_{ \pm}\right) \in L^{2}\left(D_{ \pm}\right)
\end{array}\right.
$$

Note that the condition $\nabla\left(w_{p}-w_{ \pm}\right) \in L^{2}\left(D_{ \pm}\right)$in (49) formalizes the property: $\nabla w_{p}$ converges to $\nabla w_{ \pm}$as $x_{1} \rightarrow \pm \infty$.

We are going to prove the following

Theorem 5.1 Assume that the two periodic structures satisfy the commensurability condition (47). Let $a(x)$ be defined by (40), (41), (42). Assume that the regularity conditions (7) and (10) (with $b=0$ ) are satisfied. Then, problem (49) has a solution in $H_{\mathrm{loc}}^{1}\left(\mathbb{R}^{d}\right)$, which is unique up to the addition of a constant. In addition, $\nabla\left(w_{p}-w_{ \pm}\right)$decays exponentially as $x_{1} \rightarrow \pm \infty$.

Remark 18 The properties of $w_{p}$ established in Theorem 5.1 of course imply that $w_{p}$ is strictly sublinear at infinity when $\left|x_{1}\right| \longrightarrow+\infty$.

In order to prove Theorem 5.1, we need a couple of technical lemmas.

Lemma 5.2 Consider $g \in W_{\mathrm{loc}}^{1,1}\left(D_{+}, \mathbb{R}^{d}\right)$ such that

(i) $g$ is $T_{i}$-periodic in $x_{i}$ for any $i \geq 2$,

(ii) g satisfies

$$
\exists R>0, \quad \forall x \in \mathbb{R}^{d} \text { s.t. } x_{1} \geq R, \quad \operatorname{div}(g)(x)=0 .
$$

(iii) $g$ is periodic with respect to $x_{1} \geq R$, that is, there exists $S_{1}>0$ such that

$$
\forall x \in \mathbb{R}^{d} \text { s.t. } x_{1}>R, \quad g\left(x_{1}+S_{1}, x_{2}, \ldots, x_{d}\right)=g\left(x_{1}, x_{2}, \ldots, x_{d}\right) .
$$

(iiii) denoting by $Q_{+}=\left[0, S_{1}\right] \times\left[0, T_{2}\right] \times \cdots \times\left[0, T_{d}\right]$, g satisfies, $\forall x \in \mathbb{R}^{d}$,

$$
x_{1}>R \Rightarrow \int_{x+Q_{+}} g=0 .
$$

Then,

$$
\forall x_{1} \geq R, \quad \int_{\left[0, T_{2}\right] \times \ldots\left[0, T_{d}\right]} g\left(x_{1}, x_{2}, \ldots, x_{d}\right) \cdot e_{1} d x_{2} \cdots d x_{d}=0 .
$$

Proof: Using a density argument, it is sufficient to prove the result for $g \in C^{1}$. Consider $R$ as defined in the Lemma, and $R^{\prime} \geq R$. Then we integrate $\operatorname{div}(g)=0$ on the set

$$
D_{R, R^{\prime}}=\left[R, R^{\prime}\right] \times\left[0, T_{2}\right] \times \cdots \times\left[0, T_{d}\right]=D \cap\left\{R \leq x_{1} \leq R^{\prime}\right\} .
$$

Using the fact that $g$ is periodic in the directions $2 \leq i \leq d$, we see that the boundary terms in those directions cancel out, whence: $0=\int_{D_{R, R^{\prime}}} \operatorname{div}(g)=\int_{D \cap\left\{x=R^{\prime}\right\}} g \cdot e_{1}-\int_{D \cap\{x=R\}} g \cdot e_{1}$. Hence, the map $R^{\prime} \mapsto \int_{D \cap\left\{x=R^{\prime}\right\}} g \cdot e_{1}$, is constant for $R^{\prime}>R$. If this constant is not 0 , then we have a contradiction with (51). This shows (52). 
Lemma 5.3 Consider $g \in L^{\infty}\left(D, \mathbb{R}^{d}\right)$ such that $\operatorname{div}(g) \in L^{\infty}, g$ is $T_{i}$-periodic in $x_{i}$ for any $i \geq 2$, the restriction of which on $D_{-}$(resp. $D_{+}$) satisfies the assumptions (i)-(ii)-(iii)-(iiii) of Lemma 5.2, thus (52). Then, the problem

$$
\left\{\begin{array}{l}
-\Delta u=\operatorname{div}(g), \\
u \text { is } T_{i}-\text { periodic in } x_{i}, \forall 2 \leq i \leq d, \\
\nabla u \in L^{2}(D),
\end{array}\right.
$$

has a solution, unique up to the addition of a constant.

Proof: We define, for $k=\left(0, k_{2}, \ldots, k_{d}\right) \in\{0\} \times \mathbb{Z}^{d-1}$,

$$
D_{k}=D+\left(0, k_{2} T_{2}, k_{3} T_{3}, \ldots, k_{d} T_{d}\right) .
$$

We introduce $u_{k}$, the convolution of $\operatorname{div}(g) \mathbf{1}_{D_{k}}$ with the Green function of the Laplacian of $\mathbb{R}^{d}$

$$
u_{k}=\int_{D_{k}} \operatorname{div}(g(y)) \frac{1}{|x-y|^{d-2}} d y
$$

so that

$$
\nabla u_{k}=\int_{D_{k}} \operatorname{div}(g(y)) \frac{x-y}{|x-y|^{d}} d y .
$$

Up to irrelevant universal constants we omit throughout this proof, $u_{k}$ solves

$$
-\Delta u_{k}=\operatorname{div}(g) \mathbf{1}_{D_{k}},
$$

the solution of which is given by (54). This convolution product exists because $\operatorname{div}(g)$ is bounded, has compact support in $x_{1}$, and $|x|^{-d+2} \in L_{\mathrm{loc}}^{1}\left(\mathbb{R}^{d}\right)$. Since the $D_{k}$ form a partition of the space, in the sense that

$$
\sum_{k \in\{0\} \times \mathbb{Z}^{d-1}} 1_{D_{k}}=1
$$

we are going to show that

$$
u=\sum_{k \in\{0\} \times \mathbb{Z}^{d-1}} u_{k}
$$

makes sense and that this function is a solution to (53). Formally, it is, by construction, a $T_{i}$-periodic function for all $2 \leq i \leq d$. The main point is to prove that the series

$$
(\nabla u=) \sum_{k \in\{0\} \times \mathbb{Z}^{d-1}} \nabla u_{k}=\sum_{k \in\{0\} \times \mathbb{Z}^{d-1}} \int_{D_{k}} \operatorname{div}(g(y)) \frac{x-y}{|x-y|^{d}} d y,
$$

converges, and that this function is in $L^{2}(D)$. On the other hand, to prove that $u$ itself is well defined as the series (55), one uses the Green formula on the integral (54) to make a series in $\frac{x-y}{|x-y|^{d-1}}$ appear, and next mimick the proof we are going to make. The property (51) then plays the role of property (52) we will use below.

In order to prove that the series (56) converges, we define, for any $k=\left(0, k_{2}, \ldots, k_{d}\right) \in\{0\} \times \mathbb{Z}^{d-1}$,

$$
k_{T}=\left(0, k_{2} T_{2}, \ldots, k_{d} T_{d}\right)
$$

and write, using the fact that $g$ is periodic in each variable except $x_{1}$,

$$
\nabla u_{k}(x)=\int_{D} \operatorname{div}(g(y)) \frac{x-y-k_{T}}{\left|x-y-k_{T}\right|^{d}} d y=\int_{D_{R}} \operatorname{div}(g(y)) \frac{x-y-k_{T}}{\left|x-y-k_{T}\right|^{d}} d y,
$$

where $R$ satisfies (50), and, as above, $D_{R}=D \cap\left\{\left|x_{1}\right|<R\right\}$. Next, we expand $\left(x-y-k_{T}\right)\left|x-y-k_{T}\right|^{-d}$ in powers of $\left|x-k_{T}\right|$ when the latter goes to infinity:

$$
\frac{x-y-k_{T}}{\left|x-y-k_{T}\right|^{d}}=\frac{x-k_{T}}{\left|x-k_{T}\right|^{d}}+O\left(\frac{1}{\left|x-k_{T}\right|^{d}}\right) \text {, }
$$


where the remainder is bounded uniformly with respect to $y \in D_{R}$. Inserting this into the expression of $\nabla u_{k}$, we find

$$
\nabla u_{k}(x)=\frac{x-k_{T}}{\left|x-k_{T}\right|^{d}} \int_{D_{R}} \operatorname{div}(g(y)) d y+O\left(\frac{1}{\left|x-k_{T}\right|^{d}}\right)
$$

where we have used the fact that $\operatorname{div}(g) \in L^{\infty}$ in order to bound the remainder term. Now, using the Green formula on $D_{R},(52)$ implies that $\int_{D_{R}} \operatorname{div}(g)=0$, so we obtain that the first term vanishes. Thus, for $\left|x-k_{T}\right|$ sufficiently large, we have

$$
\left|\nabla u_{k}(x)\right| \leq \frac{C}{\left|x-k_{T}\right|^{d}},
$$

for a constant $C$ that does not depend on $x-k_{T}$. This proves the convergence of the series (56). We now prove that this defines a function in $L^{2}(D)$. We split $x$ into two components : $x_{1}$ and $x^{\prime}=\left(0, x_{2}, \ldots, x_{d}\right) \in$ $\{0\} \times \mathbb{R}^{d-1}$, and observe, for all $k$, that

$$
\left|x-k_{T}\right| \geq\left|x_{1}\right|, \quad \text { and } \quad\left|x-k_{T}\right| \geq\left|x^{\prime}-k_{T}\right|,
$$

so that

$$
\left|x-k_{T}\right|^{d} \geq\left|x_{1}\right|^{\alpha d}\left|x^{\prime}-k_{T}\right|^{(1-\alpha) d}
$$

where $\alpha \in[0,1]$ will be chosen below. Hence,

$$
\sum_{\substack{k \in\{0\} \times \mathbb{Z}^{d-1} \\\left|x-k_{T}\right| \text { large }}}\left|\nabla u_{k}(x)\right| \leq C \frac{1}{\left|x_{1}\right|^{\alpha d}} \sum_{\substack{k \in\{0\} \times \mathbb{Z}^{d-1} \\\left|x-k_{T}\right| \text { large }}} \frac{1}{\left|x^{\prime}-k_{T}\right|^{(1-\alpha) d}} .
$$

We now choose $\alpha$ such that $(1-\alpha) d>d-1$, so that the sum converges, and $\alpha d>1 / 2$, so that $\left|x_{1}\right|^{2 \alpha d}$ is integrable at infinity. These constraints are equivalent to

$$
\frac{1}{d}>\alpha>\frac{1}{2 d}
$$

Using this value of $\alpha$, we have proved that the right-hand side of $(57)$ is in $L^{2}(D)$. The terms corresponding to indices $k$ such that $\left|x-k_{T}\right|$ is not large are simply addressed upon noticing directly on the expression of $\nabla u_{k}$ that

$$
\forall x \in D_{R}^{C}, \quad\left|\nabla u_{k}(x)\right| \leq \frac{C}{|x|^{d-1}},
$$

so that $\nabla u_{k} \in L^{2}(D)$.

It remains to prove uniqueness. This amounts to proving that if $u$ satisfies (53) with $g=0$, then $u$ is constant. Put differently, if $u$ is harmonic, periodic in $x_{i}$ for all $i \geq 2$ and $\nabla u \in L^{2}(D)$, then $u$ is constant. This is easily performed either by making a simple proof using Fourier series, or by applying our argument of Step 4 of the proof of Theorem 5.1 below.

We are now in position to give the proof of Theorem 5.1.

Proof of Theorem 5.1: The outline of the proof is as follows. In order to solve (49), we first write this equation in the form (59) below, namely

$$
-\operatorname{div}(a \nabla \tilde{w})=\operatorname{div}(f)+\operatorname{div}(g),
$$

where $f$ and $g$ have appropriate properties, for an unknown function $\tilde{w}$ that is essentially defined as $\tilde{w}=$ $w_{p}-\left(w_{-}, w_{+}\right)$(see the correct expression in (58) below). The point (which is the purpose of Step 3 below) is to first solve that equation (59) for $a \equiv 1$ and $f \equiv 0$ (the term arising from the presence of $f$ is indeed simpler, and addressed separately). This is where we use Lemma 5.3 and the properties of $g$ : the fact that we manipulate the Laplacian operator allows us to prove existence of a solution $u$ by using explicit convolution formulae for the solution (as seen in the proof of Lemma 5.3 above). Next, we reinstate the value of $a$ and solve

$$
-\operatorname{div}(a \nabla \tilde{w})=-\Delta u,
$$


using the Lax-Milgram Theory.

Let us make this precise. We proceed in several steps.

Step 1: subtracting the correctors $w_{+}$and $w_{-}$. As announced, we decompose as follows the solution $w_{p}$ to (49) we seek: we define

$$
\tilde{w}=w_{p}-\chi_{+} w_{+}-\chi_{-} w_{-},
$$

where the correctors $w_{ \pm}$are the periodic correctors defined in (45)-(46) and $\chi_{ \pm} \geq 0$ are smooth, bounded, nonnegative cut-off functions depending only on $x_{1}$ such that

$$
\chi_{+}(x)=\left\{\begin{array}{ll}
1 & \text { if } x_{1}>1, \\
0 & \text { if } x_{1}<0,
\end{array} \quad \chi_{-}(x)=\left\{\begin{array}{ll}
0 & \text { if } x_{1}>0 \\
1 & \text { if } x_{1}<-1
\end{array} .\right.\right.
$$

Then we have:

$$
\begin{gathered}
\nabla w_{p}=\nabla \tilde{w}+\chi_{+} \nabla w_{+}+\chi_{-} \nabla w_{-}+w_{+} \nabla \chi_{+}+w_{-} \nabla \chi_{-} . \\
a\left(\nabla w_{p}+p\right)=a \nabla \tilde{w}+a_{+} \chi_{+}\left(\nabla w_{+}+p\right)+a_{-} \chi_{-}\left(\nabla w_{-}+p\right) \\
+a p\left(1-\chi_{+}-\chi_{-}\right)+a_{+} w_{+} \nabla \chi_{+}+a_{-} w_{-} \nabla \chi_{-} .
\end{gathered}
$$

Hence (49) writes as

$$
\begin{aligned}
\operatorname{div}\left(a^{*} p\right)=\operatorname{div}\left(a\left(\nabla w_{p}+p\right)\right)=\operatorname{div}(a \nabla \tilde{w}) & +\operatorname{div}\left(a_{+} \chi_{+}\left(\nabla w_{+}+p\right)\right)+\operatorname{div}\left(a_{-} \chi_{-}\left(\nabla w_{-}+p\right)\right) \\
& +\operatorname{div}\left(a p\left(1-\chi_{+}-\chi_{-}\right)\right)+\operatorname{div}\left(a_{+} w_{+} \nabla \chi_{+}\right)+\operatorname{div}\left(a_{-} w_{-} \nabla \chi_{-}\right),
\end{aligned}
$$

that is,

$$
\begin{aligned}
-\operatorname{div}(a \nabla \tilde{w})=\operatorname{div}\left[\left(a-a^{*}\right) p\left(1-\chi_{+}-\chi_{-}\right)\right. & \left.+a_{+} w_{+} \nabla \chi_{+}+a_{-} w_{-} \nabla \chi_{-}\right] \\
& +\operatorname{div}\left[\chi_{+}\left(a_{+}\left(\nabla w_{+}+p\right)-a_{+}^{*} p\right)+\chi_{-}\left(a_{-}\left(\nabla w_{-}+p\right)-a_{-}^{*} p\right)\right] .
\end{aligned}
$$

This equation may be written in the more concise form,

$$
-\operatorname{div}(a \nabla \tilde{w})=\operatorname{div}(f)+\operatorname{div}(g),
$$

where

$$
f=\left(a-a^{*}\right) p\left(1-\chi_{+}-\chi_{-}\right)+a_{+} w_{+} \nabla \chi_{+}+a_{-} w_{-} \nabla \chi_{-}
$$

and

$$
g=\chi_{+}\left(a_{+}\left(\nabla w_{+}+p\right)-a_{+}^{*} p\right)+\chi_{-}\left(a_{-}\left(\nabla w_{-}+p\right)-a_{-}^{*} p\right)
$$

are both periodic in $x_{i}$ for any $i \geq 2$. In addition, because of the properties of the cut-off functions $\chi_{ \pm}$,

$$
f \text { has compact support in } x_{1} \text {, }
$$

and, because of (45)-(46),

$$
g \in C^{0, \alpha}, \quad \operatorname{div}(g) \text { has compact support in } x_{1} .
$$

Note that $f$ is not continuous since $a^{*}$ and $a$ are discontinuous, and that $g$ is not compactly supported in $x_{1}$ (except in the trivial case when $a$ is constant). Equation (59) being linear, we can solve it separately for the right hand sides in $f$ and $g$. This will give a solution to (59).

Step 2: solution of (59) with $g=0$. We wish to solve equation (59) with $g=0$, namely

$$
-\operatorname{div}(a \nabla u)=\operatorname{div}(f) .
$$

Here, we have $f \in L^{2}(D)^{d}$, periodic in $x_{i}$ for all $i \geq 2$. We define

$$
\mathcal{H}=\mathcal{G} / \mathbb{R}, \quad \text { with } \quad \mathcal{G}=\left\{u \in H_{\text {loc }}^{1}\left(\mathbb{R}^{d}\right), \quad u T_{i} \text { - periodic in } x_{i}, \quad \forall 2 \leq i \leq d, \quad u \in H^{1}(D)\right\} .
$$


This space is a Hilbert space for the scalar product defined by

$$
\forall(u, v) \in \mathcal{H}^{2}, \quad\langle u \mid v\rangle=\int_{D} \nabla u \cdot \nabla v
$$

In that space $\mathcal{H}$, equation (60) is equivalent to the weak formulation

$$
\forall v \in \mathcal{H}, \quad \int_{D}(a \nabla u) \cdot \nabla v=\int_{D} f \nabla v
$$

Since $a$ is elliptic and bounded, the left-hand side is a continuous coercive bilinear form on $\mathcal{H}$, and since $f \in L^{2}$, the right-hand side is a continuous linear form on $\mathcal{H}$. Applying Lax-Milgram's lemma, (60) has a unique solution in $\mathcal{H}$, that is, up to the addition of a constant.

Note that, actually in step 2, we did not use the fact that $f$ has compact support, but only that $f \in L^{2}(D)^{d}$.

Step 3: solution of (59) with $f=0$. Note that, in general, $g \notin L^{2}$, so we need to use in this Step 3 a different strategy from Step 2. In order to solve (59) with $f=0$, we first solve the equation

$$
-\Delta v=\operatorname{div}(g),
$$

with periodic boundary conditions in $x_{i}$, for $i \geq 2$. Indeed, $g$ satisfies the assumptions of Lemma 5.2 and Lemma 5.3. Hence, it satisfies (52). We may thus apply Lemma 5.3, which implies that $v$ exists and satisfies $\nabla v \in L^{2}(D)$. Precisely since $\nabla v \in L^{2}(D)$, we next solve the equation

$$
-\operatorname{div}(a \nabla u)=-\Delta v
$$

with the same strategy as in Step 2, that is, by a simple application of the Lax-Milgram Lemma.

Adding the solutions obtained in steps 2 and 3, and using the linearity of the equation, we obtain a solution to (49).

Step 4: uniqueness. Proving uniqueness amounts to proving that if

$$
\operatorname{div}(a \nabla w)=0
$$

with periodic boundary condition in $x_{i}$ for $i \geq 2$ and $\nabla w \in L^{2}(D)$, then $w$ is a constant.

We first note that, according to [18, Theorem 8.32], the fact that $a \in C^{0, \alpha}$ implies that $w \in C^{1, \alpha}$. Next, using the same argument based on the integration over the domain and the application of the Green formula performed in the proof of Lemma 5.2, we have

$$
\forall x \in \mathbb{R}, \quad \int_{D \cap\left\{x_{1}=0\right\}}(a \nabla w) \cdot e_{1}=\int_{D \cap\left\{x_{1}=x\right\}}(a \nabla w) \cdot e_{1} .
$$

Now, since $\nabla w \in L^{2}(D)$, this constant must be 0 . Hence,

$$
\forall x \in \mathbb{R}, \quad \int_{D \cap\left\{x_{1}=x\right\}}(a \nabla w) \cdot e_{1}=0 .
$$

We multiply (61) by $w$ and integrate over the domain $D_{R}=D \cap\left\{\left|x_{1}\right|<R\right\}$. Using the periodicity in the transverse directions, we find

$$
\int_{D_{R}}(a \nabla w) \cdot \nabla w=\int_{D \cap\left\{x_{1}=R\right\}} w(a \nabla w) \cdot e_{1}-\int_{D \cap\left\{x_{1}=-R\right\}} w(a \nabla w) \cdot e_{1} .
$$

Using (62), we see that, in each term of the right-hand side, we can add to $w$ a function independent of $\left(x_{2}, x_{3}, \ldots, x_{d}\right)$. Hence, we have

$$
\int_{D_{R}}(a \nabla w) \cdot \nabla w=\int_{D \cap\left\{x_{1}=R\right\}}\left(w-C_{R}\right)(a \nabla w) \cdot e_{1}-\int_{D \cap\left\{x_{1}=-R\right\}}\left(w-C_{-R}\right)(a \nabla w) \cdot e_{1} .
$$


We choose $C_{R}$ and $C_{-R}$ to be the average of $w$ over the set $D \cap\left\{x_{1}=R\right\}$ and $D \cap\left\{x_{1}=-R\right\}$, respectively. Then, successively applying the Cauchy-Schwarz and the Poincaré-Wirtinger inequality, and using that $|\nabla w|^{2} \geq \sum_{2 \leq i \leq d}\left|\nabla_{x_{i}} w\right|^{2}$, we find that

$$
\int_{D_{R}}(a \nabla w) \cdot \nabla w \leq C \int_{D \cap\left\{\left|x_{1}\right|=R\right\}}|\nabla w|^{2} .
$$

Hence, using that $a$ is elliptic, we have, denoting, for $R \geq 0, F(R)=\int_{D_{R}}|\nabla w|^{2}$,

$$
F(R) \leq C F^{\prime}(R),
$$

for some constant $C>0$ independent of $R$. Thus, if $F$ is not 0 , it grows exponentially as $R \rightarrow+\infty$.

Step 5: exponential decay. It remains to prove that $\nabla \tilde{w}$ decays exponentially as $\left|x_{1}\right| \rightarrow+\infty$. For this purpose, we note that $\operatorname{div}(a \nabla \tilde{w})$ has compact support in $x_{1}$. Hence, defining $R_{0}>0$ such that $\operatorname{supp}(\operatorname{div}(a \nabla \tilde{w})) \subset$ $\left\{\left|x_{1}\right|<R_{0}\right\}$, we pick $R^{\prime}>R>R_{0}$ and integrate (59) on $D_{R, R^{\prime}}=D \cap\left\{R<x_{1}<R^{\prime}\right\}$. Arguing as above, we find that $\tilde{w}$ satisfies $(62)$ for $x>R_{0}$.

Next, we multiply (59) by $\tilde{w}$ and integrate over $D_{R, R^{\prime}}$. We find

$$
\int_{D_{R, R^{\prime}}}(a \nabla \tilde{w}) \cdot \nabla \tilde{w}=\int_{D \cap\left\{x_{1}=R^{\prime}\right\}} \tilde{w}(a \nabla \tilde{w}) \cdot e_{1}-\int_{D \cap\left\{x_{1}=R\right\}} \tilde{w}(a \nabla \tilde{w}) \cdot e_{1} .
$$

Applying (62), and arguing as in Step 4, we get

$$
c \int_{D_{R, R^{\prime}}}|\nabla \tilde{w}|^{2} \leq \int_{D_{R, R^{\prime}}}(a \nabla \tilde{w}) \cdot \nabla \tilde{w} \leq C \int_{D \cap\left\{x_{1}=R^{\prime}\right\}}|\nabla \tilde{w}|^{2}+C \int_{D \cap\left\{x_{1}=R\right\}}|\nabla \tilde{w}|^{2} .
$$

Since $\nabla \tilde{w} \in L^{2}(D)$, one can find a sequence $R_{n}^{\prime} \rightarrow+\infty$ such that

$$
\int_{D \cap\left\{x_{1}=R_{n}^{\prime}\right\}}|\nabla \tilde{w}|^{2} \underset{n \rightarrow+\infty}{\longrightarrow} 0
$$

Thus, taking $R^{\prime}=R_{n}^{\prime}$ in (63), and letting $n \rightarrow+\infty$, we obtain

$$
\int_{D_{R,+\infty}}|\nabla \tilde{w}|^{2} \leq C \int_{D \cap\left\{x_{1}=R\right\}}|\nabla \tilde{w}|^{2},
$$

where we have denoted by $D_{R,+\infty}$ the set $D \cap\left\{x_{1}>R\right\}$. Hence, defining

$$
F(R)=\int_{D_{R,+\infty}}|\nabla \tilde{w}|^{2},
$$

we obtain

$$
\forall R>R_{0}, \quad F^{\prime}(R)+\frac{1}{C} F(R) \leq 0 .
$$

This implies that $F$ decays exponentially as $R \rightarrow+\infty$. In particular, we have

$$
\exists C, \delta>0, \quad \forall R>0, \quad \int_{D \cap\left\{R<\left|x_{1}\right|<R+1\right\}}|\nabla \tilde{w}|^{2} \leq C e^{-\delta R} .
$$

Using standard elliptic regularity results [17, Theorem 3.2, page 88], we thus have that $|\nabla \tilde{w}|$ decays exponentially as $x_{1} \rightarrow+\infty$.

Repeating the same argument for $x_{1}<0$, we conclude the proof.

Remark 19 It is possible to extend the result of Theorem 5.1 to the case where a local defect is superimposed, that is the case of a coefficient $a=a_{0}+b$, where $a_{0}$ satisfies (40), (41) and (42), and $b \not \equiv 0 \in L^{2}\left(\mathbb{R}^{d}\right)$. We again have to assume (7) and (10). If $b \in L^{p}\left(\mathbb{R}^{d}\right)$ for some $p \leq 2$, then in fact $b \in L^{2}\left(\mathbb{R}^{d}\right)$. Our proof easily carries over to that case and the results of Theorem 5.1 are valid. Indeed, one then considers the system for $b \equiv 0$ as the reference background, and the "defect" $b$ is then addressed by a combination of our proof in [10] and by the proof above. If $p>2$, the situation is less simple. An adaptation of the proof of Section 4 is in order. Although we have not checked all the details, we believe the conclusions of Theorem 5.1 again hold. 


\subsection{Bicrystals and quasiperiodicity}

In this section, we drop the assumption (47) of commensurability of the periods. We will put ourselves in the other "extreme" setting where

$$
\forall 2 \leq i \leq d, \quad \frac{S_{i}}{R_{i}} \notin \mathbb{Q} .
$$

This is done without loss of generality. Indeed, if not all the quotients $\frac{S_{i}}{R_{i}}, i \geq 2$, are irrational and some of them are indeed rational, then a combination of the proof of this section with some ingredients of the proof of Section 5.2 allows to conclude. As we shall see below, our strategy of proof to address the quasiperiodic character of the problem that arises from the non commensurability $\frac{S_{i}}{R_{i}} \notin \mathbb{Q}$ in (64) consists in considering the problem as the trace of a periodic problem in a space of higher dimension. In that process, if some of the directions correspond to rational quotients $\frac{S_{i}}{R_{i}}$, they are easily accounted for using arguments of the preceding section. This will be clear to the reader at the end of this Section. An adaptation of our main result, Theorem 5.7 below, therefore still holds.

We define $a^{*}, a_{ \pm}^{*}$ and $w_{ \pm}$from (43), (44), (45) and (46) as above. We define

$$
D_{+}=\mathbb{R}^{+} \times\left[0, S_{2}\right] \times \cdots \times\left[0, S_{d}\right], \quad D_{-}=\mathbb{R}^{-} \times\left[0, R_{2}\right] \times \cdots \times\left[0, R_{d}\right] .
$$

similarly to our definition (48) above.

The corrector problem we now want to solve reads

$$
\left\{\begin{array}{l}
-\operatorname{div}\left(a\left(\nabla w_{p}+p\right)\right)=-\operatorname{div}\left(a^{*} p\right), \\
\limsup _{R \rightarrow+\infty}\left(\frac{1}{R^{d-1}} \int_{\left|x^{\prime}\right| \leq R} \int_{0}^{+\infty}\left|\nabla\left(w_{p}-w_{+}\right)\left(x_{1}, x^{\prime}\right)\right|^{2} d x_{1} d x^{\prime}\right)<+\infty \\
\limsup _{R \rightarrow+\infty}\left(\frac{1}{R^{d-1}} \int_{\left|x^{\prime}\right| \leq R} \int_{-\infty}^{0}\left|\nabla\left(w_{p}-w_{-}\right)\left(x_{1}, x^{\prime}\right)\right|^{2} d x_{1} d x^{\prime}\right)<+\infty
\end{array}\right.
$$

We will actually establish the quasiperiodicity of $\nabla w_{p}$ in the directions $x^{\prime}$. Therefore, the integrability conditions in (65) formally imply the sub-linearity of $w_{p}$ in all directions, as stated in (5). This will be indeed proved below.

The result we will establish below sensitively depends upon the nature of the irrational numbers $\frac{S_{i}}{R_{i}}$. We recall the following notion.

Definition 5.4 The real number $x \in \mathbb{R}$ is said to be a Liouville-Roth number if, for any $\alpha \in \mathbb{N}$, there exists $k_{\alpha} \in \mathbb{Z}$ and $j_{\alpha} \in \mathbb{N}^{*}$ such that

$$
0<\left|x-\frac{k_{\alpha}}{j_{\alpha}}\right| \leq \frac{1}{\left(j_{\alpha}\right)^{\alpha}}
$$

Consequently, if $x$ is not a Liouville-Roth number, there exists $\alpha>0$ such that

$$
\forall(k, j) \in \mathbb{Z} \times \mathbb{N}^{*}, \quad\left|x-\frac{k}{j}\right| \geq \frac{1}{j^{\alpha}} .
$$

We recall a few results about Liouville-Roth numbers, which may be found in [16]. First, a Liouville-Roth number cannot be rational. Second, for any irrational number, there exists infinitely many $(k, j) \in \mathbb{Z} \times \mathbb{N}^{*}$ such that $0<|x-k / j| \leq j^{-2}$. As a consequence, we can always take $\alpha \geq 2$.

Intuitively, Liouville-Roth numbers are numbers well approximated by rational numbers. They form a set of zero Lebesgue measure. The notion is in particular useful when it comes to deriving, for quasiperiodic functions, extensions of Poincaré-Wirtinger type inequalities for periodic functions. By definition (see more details in $[13,26,32])$, quasiperiodic functions are traces of periodic functions of a higher dimensional space, and we will use this definition throughout our text. Poincaré-Wirtinger type inequalities are generically wrong for quasiperiodic functions, but they admit some generalizations modulo some adaptation. The following Lemma 5.5 makes precise a Poincaré-Wirtinger type inequality for periodic functions under a condition that 
relates to Liouville-Roth numbers. Intuitively, taking the trace $x=y$, one can deduce from this Lemma that a Poincaré-Wirtinger type inequality holds for certain quasiperiodic functions, but it comes at the price of losing some derivatives (Note that the right-hand side of (66) is an $H^{s}$ norm, for $s \geq 1$ and not an $L^{2}$ norm as in the classical Poincaré-Wirtinger inequality). This type of result is also known as Gårding inequality, or weak Poincaré inequality in the literature. In particular, it has been used by Kozlov in [25] and more recently by Gloria and Habibi [20].

For simplicity, we state Lemma 5.5 in the particular case of a periodic function of the two-dimensional plane. The result of course applies to a higher-dimensional setting, and we will actually use it below in that setting.

Lemma 5.5 Consider two positive numbers $R>0$ and $S>0$, and assume that $R / S$ is not a Liouville-Roth number, in the sense of Definition 5.4. Denote by $Q=[0, R] \times[0, S]$. Then, there exists $s \in \mathbb{R}$ (the proof actually shows that $s \geq 1$ ) and $C>0$ such that, for any $Q$-periodic function $h=h(x, y) \in L_{\mathrm{loc}}^{2}\left(\mathbb{R}^{2}\right)$,

$$
\left\|h-\frac{1}{|Q|} \int_{Q} h\right\|_{L^{2}(Q)}^{2} \leq C\left\|\partial_{\frac{x+y}{2}} h\right\|_{H^{s}(Q)}^{2}
$$

Proof of Lemma 5.5: We use the expression of the periodic function $h$ in terms of its Fourier coefficients. We classically have (up to irrelevant universal constants)

$$
\begin{gathered}
\left\|h-\frac{1}{|Q|} \int_{Q} h\right\|_{L^{2}(Q)}^{2}=\sum_{(k, j) \in \mathbb{Z}^{2} \backslash\{(0,0)\}}\left|\widehat{h}_{k, j}\right|^{2}, \\
\left\|\partial_{\frac{x+y}{2}} h\right\|_{H^{s}(Q)}^{2}=\sum_{(k, j) \in \mathbb{Z}^{2}}\left(1+k^{2}+j^{2}\right)^{s}\left|\frac{k}{R}+\frac{j}{S}\right|^{2}\left|\widehat{h}_{k, j}\right|^{2},
\end{gathered}
$$

for all $s \in \mathbb{R}$, Since $R / S$ is not a Liouville-Roth number, there exists $\alpha \geq 2$ such that

$$
\left|\frac{k}{R}+\frac{j}{S}\right|=\frac{|j|}{R}\left|\frac{k}{j}+\frac{R}{S}\right| \geq \frac{|j|}{R} \frac{1}{|j|^{\alpha}}=\frac{1}{R}|j|^{1-\alpha} .
$$

Moreover, the fact that $R / S$ is not a Liouville-Roth number implies that $S / R$ is not a Liouville-Roth number either. Hence, there exists $\alpha^{\prime} \geq 2$ such that

$$
\left|\frac{k}{R}+\frac{j}{S}\right|=\frac{|k|}{S}\left|\frac{S}{R}+\frac{j}{k}\right| \geq \frac{|k|}{S} \frac{1}{|k|^{\alpha^{\prime}}}=\frac{1}{S}|k|^{1-\alpha^{\prime}} .
$$

Hence, setting $s=\max \left(\alpha-1, \alpha^{\prime}-1\right)$,

$$
\left\|\partial_{\frac{x+y}{2}} h\right\|_{H^{s}(Q)}^{2} \geq \sum_{(k, j) \neq(0,0) \in \mathbb{Z}^{2}}\left(\frac{1}{2 R}|j|^{2-2 \alpha}+\frac{1}{2 S}|k|^{2-2 \alpha^{\prime}}\right)\left(1+k^{2}+j^{2}\right)^{s}\left|\widehat{h}_{k, j}\right|^{2} \geq C\left\|h-\widehat{h}_{0,0}\right\|_{L^{2}(Q)}^{2},
$$

which concludes the proof.

Remark 20 Since $\alpha \geq 2$ and $\alpha^{\prime} \geq 2$, it is clear in the above proof that $s \geq 1$.

We now state an elementary result which will be used in the proof of Theorem 5.7 below.

Lemma 5.6 Assume that $w \in C^{1} \cap L^{\infty}(\mathbb{R})$ has a quasiperiodic derivative

$$
w^{\prime}(x)=V(x, x), \quad V \in C^{0}\left(\mathbb{R}^{2}\right),
$$

where $V$ is $(R, S)$ periodic in $\mathbb{R}^{2}$ and $R$ and $S$ satisfy the hypotheses of Lemma 5.5. Then, $w$ is quasiperiodic with the same quasiperiods as $w^{\prime}$, that is, $w(x)=v(x, x)$, where $v$ is $(R, S)$ periodic in $\mathbb{R}^{2}$. 
Proof: Using a density argument, we only need to prove the result for $V \in C^{\infty}$. In such a case, we can use a Fourier decomposition of $V$ :

$$
V(x, y)=\sum_{(j, k) \in \mathbb{Z}^{2}} c_{k, j} e^{2 i \pi\left(\frac{j x}{R}+\frac{k y}{S}\right)} .
$$

Using that $V$ is smooth, this series converges, since we have

$$
\forall s \geq 0, \quad \exists C_{s}>0, \quad\left|c_{k, j}\right| \leq \frac{C_{s}}{|k|^{s}+|j|^{s}}
$$

We define

$$
W(x, y)=\sum_{(j, k) \in \mathbb{Z}^{2} \backslash\{0,0\}} \frac{c_{k, j}}{2 i \pi\left(\frac{j}{R}+\frac{k}{S}\right)} e^{2 i \pi\left(\frac{j x}{R}+\frac{k y}{S}\right)} .
$$

The sum in (67) converges due to the fact that $R / S$ is not a Liouville-Roth number. Indeed, this implies that there exists $\alpha \geq 2$ such that (see the proof of Lemma 5.5)

$$
\left|\frac{k}{S}+\frac{j}{R}\right| \geq C\left(|k|^{1-\alpha}+|j|^{1-\alpha}\right) .
$$

Hence, the coefficients in (67) are bounded by

$$
\left|\frac{c_{k, j}}{2 i \pi\left(\frac{j}{R}+\frac{k}{S}\right)}\right| \leq C_{s} \frac{|k|^{1-\alpha}+|j|^{1-\alpha}}{|k|^{s}+|j|^{s}} .
$$

Since this is valid for any $s$, taking $s>2-\alpha$ gives uniform convergence. Hence, $W$ is well-defined and continuous, and is periodic with the same periods as $V$. Moreover, we clearly have $w(x)=W(x, x)-c_{0,0}$, which concludes the proof.

Our main result in this Section is the following.

Theorem 5.7 Let a be defined by (40), (41), (42). Assume that (7) (with $b=0$ ) is satisfied, and that, instead of the $C^{0, \alpha}$ regularity assumption (10), $a \in C^{\infty}$. Assume that the periods $R_{i}$ and $S_{i}, 2 \leq i \leq d$, satisfy (64). Then, problem (65) has a solution $w_{p} \in H_{\mathrm{loc}}^{1}\left(\mathbb{R}^{d}\right)$. This solution is strictly sublinear at infinity in the direction $\left|\left(x_{2}, \ldots, x_{d}\right)\right| \longrightarrow+\infty$. It is unique (up to the addition of a constant) among the solutions $w$ such that, for all $x_{1} \in \mathbb{R}, \nabla w\left(x_{1}, x_{2}, \ldots, x_{d}\right)$ is quasiperiodic in $x^{\prime}=\left(x_{2}, \ldots, x_{d}\right)$, that is,

$$
\nabla w\left(x_{1}, x^{\prime}\right)=V\left(x_{1}, x^{\prime}, x^{\prime}\right), \quad \text { with } \quad\left(x^{\prime}, y^{\prime}\right) \mapsto V\left(x_{1}, x^{\prime}, y^{\prime}\right) \quad(R, S)-\text { periodic. }
$$

If, in addition,

$$
\forall i \geq 2, \quad \frac{R_{i}}{S_{i}} \text { is not a Liouville-Roth number. }
$$

then the solution $w_{p}$ itself is a bounded quasiperiodic function in the directions $2 \leq i \leq d$.

Remark 21 The assumption $a \in C^{\infty}$ is not necessary. We need that the gradient of the solution to system (102) be continuous, so that we can take its trace on the subspace $x^{\prime}=y^{\prime}$. Hence, $a \in C^{d, \alpha}$ for some $\alpha>0$, is sufficient.

Before getting to the details of the proof, let us outline the argument.

The bottom line of the argument is motivated by the quasiperiodic nature of the problem that arises at the interface. The corrector problem (65) we consider may be formally seen as two coupled problems set in $D_{+}$on the one hand, and in $D_{-}$on the other hand:

$$
\left\{\begin{array}{l}
-\operatorname{div}\left(a_{-}\left(\nabla w_{p}+p\right)\right)=0 \text { in }\left\{x_{1}<0\right\}, \\
-\operatorname{div}\left(a_{+}\left(\nabla w_{p}+p\right)\right)=0 \text { in }\left\{x_{1}>0\right\},
\end{array}\right.
$$

where we have used the fact that $a^{*}$ is constant in each half-space $\left\{x_{1}>0\right\}$ and $\left\{x_{1}<0\right\}$. Since $a^{*}$ is discontinuous across the interface $\left\{x_{1}=0\right\}$, we have, in the sense of distributions,

$$
\operatorname{div}\left(a^{*} p\right)=\left[\left(a_{+}^{*}-a_{-}^{*}\right) p\right] \cdot e_{1} \delta_{\left\{x_{1}=0\right\}}
$$


Hence, we infer from (65) a coupling condition across the interface:

$$
a_{-}\left(\nabla w_{p}\left(x_{1}=0^{-}\right)+p\right) \cdot e_{1}=a_{+}\left(\nabla w_{p}\left(x_{1}=0^{+}\right)+p\right) \cdot e_{1}-\left[\left(a_{+}^{*}-a_{-}^{*}\right) p\right] \cdot e_{1} .
$$

Since the two periodic structures on the two sides of the interface do not share a common periodic pattern along the directions of that interface (as they used to in Section 5.2), the transmission conditions (70) across the interface are in essence quasiperiodic. The periodic structure on the half space $x_{1}<0$ is incompatible with that for $x_{1}>0$, and quasiperiodicity indeed follows. Now, quasiperiodicity can be seen as periodicity in a higher dimension (this is actually its very definition). Therefore, it is natural that, at some stage, the proof goes by lifting the original corrector problem in a higher dimensional space, so as to transform quasiperiodicity into periodicity along the directions of the interface. Reinstating periodicity brings up back, to some vague extent, to the setting of Section 5.2. A similar strategy was used by Kozlov in [25] to study homogenization in an almost periodic setting. We will return to this in Remark 25. Along that general outline, some details have to be worked out. The periodic problem obtained upon lifting the quasiperiodic problem in a higher dimension is, by construction, degenerate. It is not elliptic, and not even hypoelliptic. We therefore need to regularize it and pass to the limit in the regularization. To obtain the solution of the regularized problem, we are going to apply the Schwarz iteration method in order to build a solution to the coupled system. The proof of the convergence of this algorithm is similar to the method exposed in [27]. This is one among several strategies of proof, and, in Remark 22, we will mention a variant that does not employ Schwarz iterations.

Proof of Theorem 5.7: The proof consists in several steps.

Step 1: two coupled problems. As we did in Section 5.2, we subtract $w_{ \pm}$to $w_{p}$ on each side of the coupled problem (69):

$$
\left\{\begin{array}{l}
w_{p}=w_{-}+\tilde{w}_{-} \text {in }\left\{x_{1}<0\right\}, \\
w_{p}=w_{+}+\tilde{w}_{+} \text {in }\left\{x_{1}>0\right\},
\end{array}\right.
$$

where $w_{ \pm}$are defined by (45) and (46), respectively. Hence, the system (69) may be written as follows:

$$
\left\{\begin{array}{l}
-\operatorname{div}\left(a_{-} \nabla \tilde{w}_{-}\right)=0 \text { in }\left\{x_{1}<0\right\} \\
\limsup _{R \rightarrow+\infty}\left(\frac{1}{R^{d-1}} \int_{\left|x^{\prime}\right| \leq R} \int_{0}^{+\infty}\left|\nabla\left(w_{p}-w_{+}\right)\left(x_{1}, x^{\prime}\right)\right|^{2} d x_{1} d x^{\prime}\right)<+\infty \\
-\operatorname{div}\left(a_{+} \nabla \tilde{w}_{+}\right)=0 \text { in }\left\{x_{1}>0\right\} \\
\limsup _{R \rightarrow+\infty}\left(\frac{1}{R^{d-1}} \int_{\left|x^{\prime}\right| \leq R} \int_{-\infty}^{0}\left|\nabla\left(w_{p}-w_{-}\right)\left(x_{1}, x^{\prime}\right)\right|^{2} d x_{1} d x^{\prime}\right)<+\infty \\
\left(a_{+} \nabla \tilde{w}_{+}\right) \cdot e_{1}=\left(a_{-} \nabla \tilde{w}_{-}\right) \cdot e_{1}+\eta \text { on }\left\{x_{1}=0\right\}
\end{array}\right.
$$

where the function

$$
\eta=\left[\left(a_{+}^{*}-a_{-}^{*}\right) p-a_{+}\left(\nabla w_{+}+p\right)+a_{-}\left(\nabla w_{-}+p\right)\right] \cdot e_{1}
$$

is defined in $\mathbb{R}^{d}$ (recall that $a_{ \pm}^{*}$ are constants and that $a_{ \pm}$are periodic) and is the sum of two periodic functions with incommensurable periods. Hence, it is a quasiperiodic function in $\mathbb{R}^{d}$.

Step 2: doubling the dimension. In order to solve the quasiperiodic problem (71), we use additional variables and lift it into a periodic problem. We introduce the following notation:

$$
\forall x \in \mathbb{R}^{d}, \quad x=\left(x_{1}, x^{\prime}\right), \text { where } x^{\prime}=\left(x_{2}, \ldots, x_{d}\right) \in \mathbb{R}^{d-1},
$$

and we define the following functions for $x \in \mathbb{R}^{d}, y^{\prime}=\left(y_{2}, \ldots, y_{d}\right) \in \mathbb{R}^{d-1}$,

$$
\begin{aligned}
& \bar{a}_{-}\left(x, y^{\prime}\right)=a_{-}\left(x_{1}, x^{\prime}\right), \\
& \bar{a}_{+}\left(x, y^{\prime}\right)=a_{+}\left(x_{1}, y^{\prime}\right),
\end{aligned}
$$

so that $a_{ \pm}(x)=\bar{a}_{ \pm}\left(x_{1}, x^{\prime}, x^{\prime}\right)$, and $\bar{a}_{ \pm}$are periodic with respect to the variable $\left(x^{\prime}, y^{\prime}\right)$, of periods $R_{2}, \ldots, R_{d}, S_{2}, \ldots, S_{d}$. We also define

$$
\bar{\eta}\left(x, y^{\prime}\right)=\left[\left(a_{+}^{*} p-a_{+}\left(\nabla w_{+}+p\right)\right) \cdot e_{1}\right]\left(x_{1}, y^{\prime}\right)-\left[\left(a_{-}^{*} p-a_{-}\left(\nabla w_{-}+p\right)\right) \cdot e_{1}\right]\left(x_{1}, x^{\prime}\right) .
$$


Moreover, the definition of $a_{ \pm}^{*}$ implies that

$$
\operatorname{div}\left[a_{+}^{*} p-a_{+}\left(\nabla w_{+}+p\right)\right]=\operatorname{div}\left[a_{-}^{*} p-a_{-}\left(\nabla w_{-}+p\right)\right]=0,
$$

and that $\langle\eta\rangle=0$. Hence, applying Lemma 5.2, we deduce that $\bar{\eta}$ has zero average on each hyperplane $x_{1}=$ Cte:

$$
\forall x_{1} \in \mathbb{R}, \quad \int \bar{\eta}\left(x_{1}, x_{2}, \cdots, x_{d}, y_{2}, \cdots, y_{d}\right) d x_{2} \cdots d x_{d} d y_{2} \cdots d y_{d}=0
$$

We also define the following differential operators on $\mathbb{R}^{2 d-1}$ :

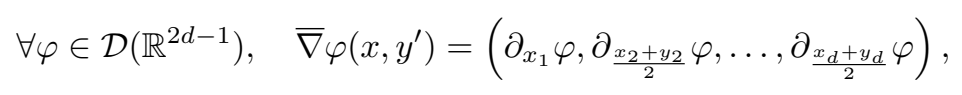

and

$$
\forall \phi \in \mathcal{D}\left(\mathbb{R}^{2 d-1}, \mathbb{R}^{d}\right), \quad \overline{\operatorname{div}}(\phi)=\partial_{x_{1}} \phi_{1}+\sum_{i=2}^{d} \frac{\partial_{x_{i}+y_{i}}}{2} \phi_{i} .
$$

It is clear that these operators have dense domain in $H^{1}$, and that they are adjoint to one another. We now consider the system

$$
\left\{\begin{array}{l}
-\overline{\operatorname{div}}\left(\bar{a}_{-} \bar{\nabla}_{\bar{w}_{-}}\right)=0 \text { in }\left\{x_{1}<0\right\} \\
\bar{\nabla} \bar{w}_{-} \in L^{2}\left(\bar{D}_{-}\right) \\
-\overline{\operatorname{div}}\left(\bar{a}_{+} \bar{\nabla} \bar{w}_{+}\right)=0 \text { in }\left\{x_{1}>0\right\} \\
\bar{\nabla}_{+} \in L^{2}\left(\bar{D}_{+}\right) \\
\left(\bar{a}_{+} \bar{\nabla}_{+}\right) \cdot e_{1}=\left(\bar{a}_{-} \bar{\nabla} \bar{w}_{-}\right) \cdot e_{1}+\bar{\eta} \text { on }\left\{x_{1}=0\right\} \\
\bar{w}_{ \pm} \text {is periodic in } x_{2}, \ldots, x_{d}, y_{2}, \ldots, y_{d}
\end{array}\right.
$$

where the domains $\bar{D}_{ \pm}$are defined by

$$
\bar{D}_{ \pm}=\mathbb{R}^{ \pm} \times\left[0, R_{2}\right] \times \cdots \times\left[0, R_{d}\right] \times\left[0, S_{2}\right] \times \cdots \times\left[0, S_{d}\right] .
$$

In the last line of (75), it is understood that, for all $2 \leq i \leq d$, the period in the variable $x_{i}$ is $R_{i}$, and the period for $y_{i}$ is $S_{i}$. If $\bar{w}_{ \pm}$is a solution to (75), then, setting

$$
\tilde{w}_{ \pm}(x)=\bar{w}_{ \pm}\left(x_{1}, x^{\prime}, x^{\prime}\right),
$$

it is clear that $\tilde{w}_{ \pm}$is a solution to (71), formally. To make it rigorous, and in particular to take the trace in (77), regularity is needed, which we will prove in the sequel.

Step 3: regularization. Problem (75) is now periodic in all variables except $x_{1}$. In that sense, it shares some similarity with the problems we considered in Section 5.2. However, and this is a sharp difference, the differential operator in (75) is degenerate. A regularization is thus in order. For this purpose, we wish to define, for any $\delta>0, \bar{w}_{\delta}=\left(\bar{w}_{\delta,-}, \bar{w}_{\delta,+}\right)$ as a solution of the problem

$$
\left\{\begin{array}{l}
-\overline{\operatorname{div}}\left(\bar{a}_{-} \bar{\nabla} \bar{w}_{\delta,-}\right)-\delta \Delta \bar{w}_{\delta,-}+\delta \bar{w}_{\delta,-}=0 \text { in }\left\{x_{1}<0\right\} \\
\bar{\nabla} \bar{w}_{\delta,-} \in L^{2}\left(\bar{D}_{-}\right) \\
-\overline{\operatorname{div}}\left(\bar{a}_{+} \bar{\nabla}_{\delta,+}\right)-\delta \Delta \bar{w}_{\delta,+}+\delta \bar{w}_{\delta,+}=0 \text { in }\left\{x_{1}>0\right\} \\
\bar{\nabla}_{\delta,+} \in L^{2}\left(\bar{D}_{+}\right), \\
\left(\bar{a}_{+} \bar{\nabla}_{\bar{w}_{\delta,+}}\right) \cdot e_{1}+\delta \nabla \bar{w}_{\delta,+} \cdot e_{1}=\left(\bar{a}_{-} \bar{\nabla} \bar{w}_{\delta,-}\right) \cdot e_{1}+\delta \nabla \bar{w}_{\delta,-} \cdot e_{1}+\bar{\eta} \\
\quad \text { on }\left\{x_{1}=0\right\} \\
\bar{w}_{\delta, \pm} \text { is periodic in } x_{2}, \ldots, x_{d}, y_{2}, \ldots, y_{d},
\end{array}\right.
$$

where we again imply that the period for the variable $x_{i}$ is $R_{i}$, and the period for $y_{i}$ is $S_{i}$, for any $i \geq 2$. The sets $\bar{D}_{ \pm}$are defined by (76). The operator $\Delta$ in (78) is the "full" laplacian, that is,

$$
\Delta=\sum_{i=1}^{d} \partial_{x_{i}}^{2}+\sum_{i=2}^{d} \partial_{y_{i}}^{2}
$$


which, of course, is strictly elliptic. Hence, at least formally, given $\bar{w}_{\delta,-}$, there exists a unique solution $\bar{w}_{\delta,+}$ to problem (78), and vice versa. The purpose of our next three steps, Steps 4 through 6 , is now to prove the existence of a solution $\left(\bar{w}_{\delta,-}, \bar{w}_{\delta,+}\right)$ to (78). Note that, strictly speaking, we do not need uniqueness (although it will be true, up to the addition of constants of course), but only existence.

Step 4: Schwarz iteration method. We apply the Schwarz iteration method in its (so-called parallel) form described in [27]. The slight difference between our setting and that of the reference [27] is that we work in a domain unbounded in one direction, but the presence of the zero order term $+\delta w$ in the equation compensates for this loss of compactness and allows us to perform very similar arguments. We only provide them here (in this step and the following two steps) for the convenience of the reader. To lighten the notation, and since throughout Steps 4 to 6 , the regularizing parameter $\delta$ is fixed, we temporarily drop the subscript $\delta$ in $\bar{w}_{\delta, \pm}$. We fix $\lambda>0$, and define the following sequence:

$$
\begin{gathered}
\left\{\begin{array}{c}
-\overline{\operatorname{div}}\left(\bar{a}_{-} \bar{\nabla} \bar{w}_{-}^{n+1}\right)-\delta \Delta \bar{w}_{-}^{n+1}+\delta \bar{w}_{-}^{n+1}=0 \text { in }\left\{x_{1}<0\right\} \\
\left(\bar{a}_{-} \bar{\nabla} \bar{w}_{-}^{n+1}\right) \cdot e_{1}+\delta \nabla \bar{w}_{-}^{n+1} \cdot e_{1}+\lambda \bar{w}_{-}^{n+1} \\
\quad=\left(\bar{a}_{+} \bar{\nabla} \bar{w}_{+}^{n}\right) \cdot e_{1}+\delta \nabla \bar{w}_{+}^{n} \cdot e_{1}+\lambda \bar{w}_{+}^{n}+\bar{\eta} \text { on }\left\{x_{1}=0\right\}, \\
\bar{w}_{-}^{n+1} \text { is periodic in } x_{2}, \ldots, x_{d}, y_{2}, \ldots, y_{d} .
\end{array}\right. \\
\left\{\begin{array}{c}
-\overline{\operatorname{div}}\left(\bar{a}_{+} \bar{\nabla} \bar{w}_{+}^{n+1}\right)-\delta \Delta \bar{w}_{+}^{n+1}+\delta \bar{w}_{+}^{n+1}=0 \text { in }\left\{x_{1}>0\right\} \\
-\left(\bar{a}_{+} \bar{\nabla} \bar{w}_{+}^{n+1}\right) \cdot e_{1}-\delta \nabla \bar{w}_{+}^{n+1} \cdot e_{1}+\lambda \bar{w}_{+}^{n+1} \\
=-\left(\bar{a}_{-} \bar{\nabla} \bar{w}_{-}^{n}\right) \cdot e_{1}-\delta \nabla \bar{w}_{-}^{n} \cdot e_{1}+\lambda \bar{w}_{-}^{n}+\bar{\eta} \text { on }\left\{x_{1}=0\right\}, \\
\bar{w}_{+}^{n+1} \text { is periodic in } x_{2}, \ldots, x_{d}, y_{2}, \ldots, y_{d} .
\end{array}\right.
\end{gathered}
$$

Here again, for all $2 \leq i \leq d$, the period is $R_{i}$ for the variable $x_{i}$, and $S_{i}$ is that for the variable $y_{i}$. The purpose of the presence of the extra parameter $\lambda>0$, inserted in the coupling conditions in (79)-(80), is to ensure the convergence of the iterations. We will see this shortly. For the moment, we notice that, precisely because of the presence of the regularizing terms in $\delta$, a simple application of Lax-Milgram lemma allows to prove that, given $\bar{w}_{ \pm}^{n}$, systems (79) and (80) have a unique solution $\bar{w}_{ \pm}^{n+1} \in H^{1}\left(\bar{D}_{ \pm}\right)$. The initial guess $\bar{w}_{ \pm}^{0}$ may be any periodic function, and we choose it here to be $\bar{w}_{ \pm}^{0}=0$.

$\underline{\text { Step 5: bounds on the sequence }}\left(\bar{w}_{ \pm}^{n}\right)_{n \in \mathbb{N}}$. We now prove, following [27], that the sequence defined by (79)(80) converges. To this end, we subtract the equation satisfied by $\bar{w}_{ \pm}^{n+1}$ to that satisfied by $\bar{w}_{ \pm}^{n-1}$, finding

$$
-\overline{\operatorname{div}}\left(\bar{a}_{-} \bar{\nabla}\left(\bar{w}_{-}^{n+1}-\bar{w}_{-}^{n-1}\right)\right)-\delta \Delta\left(\bar{w}_{-}^{n+1}-\bar{w}_{-}^{n-1}\right)+\delta\left(\bar{w}_{-}^{n+1}-\bar{w}_{-}^{n-1}\right)=0 \quad \text { in }\left\{x_{1}<0\right\},
$$

with the boundary condition

$$
\bar{a}_{-} \bar{\nabla}\left(\bar{w}_{-}^{n+1}-\bar{w}_{-}^{n-1}\right) \cdot e_{1}+\delta \nabla\left(\bar{w}_{-}^{n+1}-\bar{w}_{-}^{n-1}\right) \cdot e_{1}=\lambda\left(2 \bar{w}_{+}^{n}-\bar{w}_{-}^{n+1}-\bar{w}_{-}^{n+1}\right) \text { on }\left\{x_{1}=0\right\} .
$$

Similarly,

$$
-\overline{\operatorname{div}}\left(\bar{a}_{+} \bar{\nabla}\left(\bar{w}_{+}^{n+1}-\bar{w}_{+}^{n-1}\right)\right)-\delta \Delta\left(\bar{w}_{+}^{n+1}-\bar{w}_{+}^{n-1}\right)+\delta\left(\bar{w}_{+}^{n+1}-\bar{w}_{+}^{n-1}\right)=0 \quad \text { in }\left\{x_{1}>0\right\},
$$

with the boundary condition

$$
-\bar{a}_{+} \bar{\nabla}\left(\bar{w}_{+}^{n+1}-\bar{w}_{+}^{n-1}\right) \cdot e_{1}-\delta \nabla\left(\bar{w}_{+}^{n+1}-\bar{w}_{+}^{n-1}\right) \cdot e_{1}=\lambda\left(2 \bar{w}_{-}^{n}-\bar{w}_{+}^{n+1}-\bar{w}_{+}^{n+1}\right) \text { on }\left\{x_{1}=0\right\} .
$$

Next, we multiply (81) by $\bar{w}_{-}^{n+1}-\bar{w}_{-}^{n-1}$ and integrate over $\bar{D}_{-}$:

$$
\begin{aligned}
\int_{\bar{D}_{-}}\left[\bar{a}_{-} \bar{\nabla}\left(\bar{w}_{-}^{n+1}-\bar{w}_{-}^{n-1}\right)\right] \cdot\left[\bar{\nabla}\left(\bar{w}_{-}^{n+1}-\bar{w}_{-}^{n-1}\right)\right]+ & \delta \int_{\bar{D}_{-}}\left|\nabla\left(\bar{w}_{-}^{n+1}-\bar{w}_{-}^{n-1}\right)\right|^{2}+\delta \int_{\bar{D}_{-}}\left(\bar{w}_{-}^{n+1}-\bar{w}_{-}^{n-1}\right)^{2} \\
& =\int_{\Gamma} \lambda\left(2 \bar{w}_{+}^{n}-\bar{w}_{-}^{n-1}-\bar{w}_{-}^{n+1}\right)\left(\bar{w}_{-}^{n+1}-\bar{w}_{-}^{n-1}\right), \quad(83)
\end{aligned}
$$

where $\Gamma$ is the set

$$
\Gamma=\{0\} \times\left[0, R_{2}\right] \times \cdots \times\left[0, R_{d}\right] \times\left[0, S_{2}\right] \times \cdots \times\left[0, S_{d}\right]
$$


Reorganizing the right-hand side of (83), we obtain

$$
\begin{aligned}
& \int_{\bar{D}_{-}}\left[\bar{a}_{-} \bar{\nabla}\left(\bar{w}_{-}^{n+1}-\bar{w}_{-}^{n-1}\right)\right] \cdot {\left[\bar{\nabla}\left(\bar{w}_{-}^{n+1}-\bar{w}_{-}^{n-1}\right)\right]+\delta \int_{\bar{D}_{-}}\left|\nabla\left(\bar{w}_{-}^{n+1}-\bar{w}_{-}^{n-1}\right)\right|^{2} } \\
&+\delta \int_{\bar{D}_{-}}\left(\bar{w}_{-}^{n+1}-\bar{w}_{-}^{n-1}\right)^{2}+\lambda \int_{\Gamma}\left(\bar{w}_{+}^{n}-\bar{w}_{-}^{n+1}\right)^{2}=\lambda \int_{\Gamma}\left(\bar{w}_{-}^{n-1}-\bar{w}_{+}^{n}\right)^{2} .
\end{aligned}
$$

Similar computations lead to

$$
\begin{aligned}
\int_{\bar{D}_{+}}\left[\bar{a}_{+} \bar{\nabla}\left(\bar{w}_{+}^{n+1}-\bar{w}_{+}^{n-1}\right)\right] \cdot[ & {\left[\bar{\nabla}\left(\bar{w}_{+}^{n+1}-\bar{w}_{+}^{n-1}\right)\right]+\delta \int_{\bar{D}_{+}}\left|\nabla\left(\bar{w}_{+}^{n+1}-\bar{w}_{+}^{n-1}\right)\right|^{2} } \\
& +\delta \int_{\bar{D}_{+}}\left(\bar{w}_{+}^{n+1}-\bar{w}_{+}^{n-1}\right)^{2}+\lambda \int_{\Gamma}\left(\bar{w}_{-}^{n}-\bar{w}_{+}^{n+1}\right)^{2}=\lambda \int_{\Gamma}\left(\bar{w}_{+}^{n-1}-\bar{w}_{-}^{n}\right)^{2} .
\end{aligned}
$$

We next add up (84) and (85), use (7), and sum over $1 \leq n \leq N$. We have a telescopic sum for the terms involving $\lambda$, so some cancellations occur. We end up with:

$$
\begin{aligned}
\sum_{1 \leq n \leq N}\left(\underline{\mu} \int_{\bar{D}_{ \pm}} \mid\right. & \left.\bar{\nabla}\left(\bar{w}_{ \pm}^{n+1}-\bar{w}_{ \pm}^{n-1}\right)\right|^{2}+\delta \int_{\bar{D}_{ \pm}}\left|\nabla\left(\bar{w}_{ \pm}^{n+1}-\bar{w}_{ \pm}^{n-1}\right)\right|^{2} \\
& \left.+\delta \int_{\bar{D}_{ \pm}}\left(\bar{w}_{ \pm}^{n+1}-\bar{w}_{ \pm}^{n-1}\right)^{2}\right)+\lambda \int_{\Gamma}\left(\bar{w}_{ \pm}^{N}-\bar{w}_{\mp}^{N+1}\right)^{2} \leq \lambda \int_{\Gamma}\left(\bar{w}_{-}^{0}-\bar{w}_{+}^{1}\right)^{2}+\left(\bar{w}_{+}^{0}-\bar{w}_{-}^{1}\right)^{2}
\end{aligned}
$$

For $N$, arbitrary, fixed, we in particular obtain that

$$
\int_{\Gamma}\left(\bar{w}_{ \pm}^{N}-\bar{w}_{\mp}^{N+1}\right)^{2} \leq C
$$

where the constant $C$ does not depend on $N$. And, letting $N$ go to infinity, we separately obtain

$$
\sum_{n \geq 1}\left(\int_{\bar{D}_{ \pm}}\left|\nabla\left(\bar{w}_{ \pm}^{n+1}-\bar{w}_{ \pm}^{n-1}\right)\right|^{2}+\int_{\bar{D}_{ \pm}}\left(\bar{w}_{ \pm}^{n+1}-\bar{w}_{ \pm}^{n-1}\right)^{2}\right)<+\infty
$$

Returning to (81), we multiply it by $w_{-}^{n+1}$ and integrate over $\bar{D}_{-}$:

$$
\begin{array}{r}
\int_{\bar{D}_{-}}\left[\bar{a}_{-} \bar{\nabla}\left(\bar{w}_{-}^{n+1}-\bar{w}_{-}^{n-1}\right)\right] \cdot \bar{\nabla} \bar{w}_{-}^{n+1}+\delta \int_{\bar{D}_{-}} \nabla\left(\bar{w}_{-}^{n+1}-\bar{w}_{-}^{n-1}\right) \cdot \nabla \bar{w}_{-}^{n+1}+\delta \int_{\bar{D}_{-}}\left(\bar{w}_{-}^{n+1}-\bar{w}_{-}^{n-1}\right) \bar{w}_{-}^{n+1} \\
=\int_{\Gamma} \lambda\left(2 \bar{w}_{+}^{n}-\bar{w}_{-}^{n-1}-\bar{w}_{-}^{n+1}\right) \bar{w}_{-}^{n+1} .
\end{array}
$$

We now use the elementary property

$$
(\alpha-\beta) \cdot \alpha=\frac{1}{2}|\alpha|^{2}+\frac{1}{2}|\alpha-\beta|^{2}-\frac{1}{2}|\beta|^{2},
$$

for any couple of vectors $\alpha$ and $\beta$. We first apply it with $\alpha=\sqrt{\bar{a}_{-}} \bar{\nabla} w_{-}^{n+1}$ and $\beta=\sqrt{\bar{a}_{-}} \bar{\nabla} w_{-}^{n-1}$, then to $\alpha=\nabla w_{-}^{n+1}$ and $\beta=\nabla \bar{w}_{-}^{n-1}$. (Note that we implicitly assume here that $a_{ \pm}$is symmetric, otherwise the argument can be easily adapted.) We also have

$$
\left(2 \bar{w}_{+}^{n}-\bar{w}_{-}^{n+1}-\bar{w}_{-}^{n-1}\right) \bar{w}_{-}^{n+1}=-\frac{1}{2}\left(\bar{w}_{-}^{n+1}\right)^{2}-\frac{1}{2}\left(\bar{w}_{-}^{n-1}\right)^{2}+\left(\bar{w}_{+}^{n}\right)^{2}-\left(\bar{w}_{-}^{n+1}-\bar{w}_{+}^{n}\right)^{2}+\frac{1}{2}\left(\bar{w}_{-}^{n+1}-\bar{w}_{-}^{n-1}\right)^{2} .
$$


This leads to

$$
\begin{gathered}
\frac{1}{2} \int_{\bar{D}_{-}}\left[a_{-} \bar{\nabla}\left(\bar{w}_{-}^{n+1}-\bar{w}_{-}^{n-1}\right)\right] \cdot \\
\cdot \bar{\nabla}\left(\bar{w}_{-}^{n+1}-\bar{w}_{-}^{n-1}\right)+\frac{1}{2} \int_{\bar{D}_{-}}\left(\bar{a}_{-} \bar{\nabla} \bar{w}_{-}^{n+1}\right) \cdot \bar{\nabla} \bar{w}_{-}^{n+1} \\
+\frac{\delta}{2} \int_{\bar{D}_{-}}\left|\nabla\left(\bar{w}_{-}^{n+1}-\bar{w}_{-}^{n-1}\right)\right|^{2}+\left|\nabla \bar{w}_{-}^{n+1}\right|^{2}+\frac{\delta}{2} \int_{\bar{D}_{-}}\left(\bar{w}_{-}^{n+1}-\bar{w}_{-}^{n-1}\right)^{2}+\left(\bar{w}_{-}^{n+1}\right)^{2} \\
+\frac{\lambda}{2} \int_{\Gamma}\left(\bar{w}_{-}^{n+1}\right)^{2}+\left(\bar{w}_{-}^{n-1}\right)^{2}+2\left(\bar{w}_{-}^{n+1}-\bar{w}_{+}^{n}\right)^{2} \\
=\frac{1}{2} \int_{\bar{D}_{-}}\left(\bar{a}_{-} \bar{\nabla} \bar{w}_{-}^{n-1}\right) \cdot \bar{\nabla} \bar{w}_{-}^{n-1}+\frac{\delta}{2} \int_{\bar{D}_{-}}\left|\nabla \bar{w}_{-}^{n-1}\right|^{2}+\left(\bar{w}_{-}^{n-1}\right)^{2}+\lambda \int_{\Gamma}\left(\bar{w}_{+}^{n}\right)^{2}+\frac{1}{2}\left(\bar{w}_{-}^{n+1}-\bar{w}_{-}^{n-1}\right)^{2} .
\end{gathered}
$$

A similar computation, using (82), gives

$$
\begin{aligned}
& \frac{1}{2} \int_{\bar{D}_{+}}\left[a_{+} \bar{\nabla}\left(\bar{w}_{+}^{n+1}-\bar{w}_{+}^{n-1}\right)\right] \cdot \bar{\nabla}\left(\bar{w}_{+}^{n+1}-\bar{w}_{+}^{n-1}\right)+\frac{1}{2} \int_{\bar{D}_{+}}\left(\bar{a}_{+} \bar{\nabla} \bar{w}_{+}^{n+1}\right) \cdot \bar{\nabla} \bar{w}_{+}^{n+1} \\
&+\frac{\delta}{2} \int_{\bar{D}_{+}}\left|\nabla\left(\bar{w}_{+}^{n+1}-\bar{w}_{+}^{n-1}\right)\right|^{2}+\left|\nabla \bar{w}_{+}^{n+1}\right|^{2}+\frac{\delta}{2} \int_{\bar{D}_{+}}\left(\bar{w}_{+}^{n+1}-\bar{w}_{+}^{n-1}\right)^{2}+\left(\bar{w}_{+}^{n+1}\right)^{2} \\
&+\frac{\lambda}{2} \int_{\Gamma}\left(\bar{w}_{+}^{n+1}\right)^{2}+\left(\bar{w}_{+}^{n-1}\right)^{2}+2\left(\bar{w}_{+}^{n+1}-\bar{w}_{-}^{n}\right)^{2} \\
&=\frac{1}{2} \int_{\bar{D}_{+}}\left(\bar{a}_{+} \bar{\nabla} \bar{w}_{+}^{n-1}\right) \cdot \bar{\nabla} \bar{w}_{+}^{n-1}+\frac{\delta}{2} \int_{\bar{D}_{+}}\left|\nabla \bar{w}_{+}^{n-1}\right|^{2}+\left(\bar{w}_{+}^{n-1}\right)^{2}+\lambda \int_{\Gamma}\left(\bar{w}_{-}^{n}\right)^{2}+\frac{1}{2}\left(\bar{w}_{+}^{n+1}-\bar{w}_{+}^{n-1}\right)^{2} .
\end{aligned}
$$

We add (87) and (88), and sum the result over $1 \leq n \leq N$. Hence

$$
\begin{gathered}
\frac{\delta}{2} \sum_{n=1}^{N}\left(\left\|\bar{w}_{-}^{n+1}-\bar{w}_{-}^{n-1}\right\|_{H^{1}\left(\bar{D}_{-}\right)}^{2}+\left\|\bar{w}_{+}^{n+1}-\bar{w}_{+}^{n-1}\right\|_{H^{1}\left(\bar{D}_{+}\right)}^{2}\right)+\frac{\delta}{2}\left(\left\|\bar{w}_{-}^{N+1}\right\|_{H^{1}\left(\bar{D}_{-}\right)}^{2}+\left\|\bar{w}_{+}^{N+1}\right\|_{H^{1}\left(\bar{D}_{+}\right)}^{2}\right) \\
+\frac{\lambda}{2} \sum_{n=1}^{N}\left(\int_{\Gamma}\left(\bar{w}_{-}^{n+1}\right)^{2}+\left(\bar{w}_{-}^{n-1}\right)^{2}+2\left(\bar{w}_{-}^{n+1}-\bar{w}_{+}^{n}\right)^{2}\right)+\frac{\lambda}{2} \sum_{n=1}^{N}\left(\int_{\Gamma}\left(\bar{w}_{+}^{n+1}\right)^{2}+\left(\bar{w}_{+}^{n-1}\right)^{2}+2\left(\bar{w}_{+}^{n+1}-w_{-}^{n}\right)^{2}\right) \\
\leq C_{0}+\frac{\lambda}{2} \sum_{n=1}^{N}\left(\int_{\Gamma} 2\left(\bar{w}_{+}^{n}\right)^{2}+\left(\bar{w}_{-}^{n+1}-\bar{w}_{-}^{n-1}\right)^{2}\right)+\frac{\lambda}{2} \sum_{n=1}^{N}\left(\int_{\Gamma} 2\left(\bar{w}_{-}^{n}\right)^{2}+\left(\bar{w}_{+}^{n+1}-\bar{w}_{+}^{n-1}\right)^{2}\right), \quad(89)
\end{gathered}
$$

where $C_{0}=C_{0}^{+}+C_{0}^{-}$, with

$$
C_{0}^{ \pm}=\frac{1}{2} \int_{\bar{D}_{ \pm}} \bar{a}_{ \pm} \bar{\nabla} w_{ \pm}^{1} \cdot \bar{\nabla} \bar{w}_{ \pm}^{1}+\bar{a}_{ \pm} \bar{\nabla} w_{ \pm}^{0} \cdot \bar{\nabla} \bar{w}_{ \pm}^{0}+\frac{\delta}{2} \int_{\bar{D}_{ \pm}}\left|\nabla \bar{w}_{ \pm}^{1}\right|^{2}+\left|\nabla \bar{w}_{ \pm}^{0}\right|^{2}+\frac{\delta}{2} \int_{\bar{D}_{ \pm}}\left(\bar{w}_{ \pm}^{1}\right)^{2}+\left(\bar{w}_{ \pm}^{0}\right)^{2}
$$

In (89), the sums involving $\int_{\Gamma}\left(\bar{w}_{ \pm}^{k}\right)^{2}$ cancel out, so that we end up with

$$
\begin{gathered}
\frac{\delta}{2} \sum_{n=1}^{N}\left(\left\|\bar{w}_{-}^{n+1}-\bar{w}_{-}^{n-1}\right\|_{H^{1}\left(\bar{D}_{-}\right)}^{2}+\left\|\bar{w}_{+}^{n+1}-\bar{w}_{+}^{n-1}\right\|_{H^{1}\left(\bar{D}_{+}\right)}^{2}\right)+\frac{\delta}{2}\left(\left\|\bar{w}_{-}^{N+1}\right\|_{H^{1}\left(\bar{D}_{-}\right)}^{2}+\left\|\bar{w}_{+}^{N+1}\right\|_{H^{1}\left(\bar{D}_{+}\right)}^{2}\right) \\
+\lambda \sum_{n=1}^{N} \int_{\Gamma}\left(\bar{w}_{-}^{n+1}-\bar{w}_{+}^{n}\right)^{2}+\left(\bar{w}_{+}^{n+1}-\bar{w}_{-}^{n}\right)^{2}+\frac{\lambda}{2} \int_{\Gamma}\left(\bar{w}_{-}^{N+1}\right)^{2}+\left(\bar{w}_{+}^{N+1}\right)^{2} \\
\leq C_{0}+\frac{\lambda}{2} \int_{\Gamma}\left(\bar{w}_{-}^{N}\right)^{2}+\left(\bar{w}_{+}^{N}\right)^{2}+\frac{\lambda}{2} \sum_{n=1}^{N} \int_{\Gamma}\left(\bar{w}_{-}^{n+1}-\bar{w}_{-}^{n-1}\right)^{2}+\left(\bar{w}_{+}^{n+1}-\bar{w}_{+}^{n-1}\right)^{2} .
\end{gathered}
$$

A trace inequality over $\bar{D}_{ \pm}$together with (86) show that the last term is bounded independently of $N$. Again using a trace theorem, we have $\left\|\bar{w}_{ \pm}^{N}\right\|_{L^{2}(\Gamma)}^{2} \leq C\left\|\bar{w}_{ \pm}^{N}\right\|_{H^{1}\left(\bar{D}_{ \pm}\right)}^{2}$, so that the second term of the right-hand side of (90) is bounded, as soon as $\lambda$ is sufficiently small (say, $\lambda C^{2}<\delta$ ).

$$
\sup _{n \in \mathbb{N}}\left\|\bar{w}_{ \pm}^{n}\right\|_{H^{1}\left(\bar{D}_{ \pm}\right)}<+\infty
$$




$$
\sum_{n \geq 1}\left\|\bar{w}_{ \pm}^{n+1}-\bar{w}_{\mp}^{n}\right\|_{L^{2}(\Gamma)}<+\infty
$$

Step 6: convergence of the sequence. The bound (92) implies that

$$
\bar{w}_{ \pm}^{n+1}-\bar{w}_{\mp}^{n} \underset{n \rightarrow+\infty}{\longrightarrow} 0 \text { in } L^{2}(\Gamma) .
$$

In addition, (91) shows that we may extract a subsequence, which we denote by $\bar{w}_{ \pm}^{\alpha(n)}$, such that

$$
\bar{w}_{ \pm}^{\alpha(n)} \underset{n \rightarrow+\infty}{\longrightarrow} \bar{w}_{ \pm}, \quad \bar{w}_{ \pm}^{\alpha(n)+1} \underset{n \rightarrow+\infty}{\longrightarrow} \bar{v}_{ \pm} \text {both in } H^{1}\left(\bar{D}_{ \pm}\right),
$$

and such that $w_{ \pm}$and $v_{ \pm}$are all periodic. This allows to pass to the limit in the equations (79)-(80). We obtain

$$
-\overline{\operatorname{div}}\left(\bar{a}_{ \pm} \bar{\nabla} \bar{w}_{ \pm}\right)-\delta \Delta \bar{w}_{ \pm}+\delta \bar{w}_{ \pm}=0 \text { in } \bar{D}_{ \pm}
$$

and exactly likewise for $v_{ \pm}$. Because of (93), we have

$$
\bar{w}_{ \pm}=\bar{v}_{\mp} \text { on } \Gamma .
$$

Using the trace inequalities again, we may also pass to the limit in the boundary conditions and obtain

$$
\left(\bar{a}_{-} \bar{\nabla}_{-}\right) \cdot e_{1}+\delta\left(\nabla \bar{v}_{-}\right) \cdot e_{1}+\lambda \bar{v}_{-}=\left(\bar{a}_{+} \bar{\nabla} \bar{w}_{+}\right) \cdot e_{1}+\delta\left(\nabla \bar{w}_{+}\right) \cdot e_{1}+\lambda \bar{w}_{+}+\bar{\eta},
$$

on $\Gamma$, thus, since $\bar{w}_{ \pm}$and $\bar{v}_{\mp}$ agree on $\Gamma$,

$$
\left(\bar{a}_{-} \bar{\nabla} \bar{v}_{-}\right) \cdot e_{1}+\delta\left(\nabla \bar{v}_{-}\right) \cdot e_{1}=\left(\bar{a}_{+} \bar{\nabla}_{+}\right) \cdot e_{1}+\delta\left(\nabla \bar{w}_{+}\right) \cdot e_{1}+\bar{\eta}, \text { on } \Gamma .
$$

We conclude this step defining

$$
\bar{w}_{\delta}(x)= \begin{cases}\bar{v}_{-}(x) & \text { if } x \in \bar{D}_{-}, \\ \bar{w}_{+}(x) & \text { if } x \in \bar{D}_{+},\end{cases}
$$

which, in view of all the above observations, is a solution to (78).

Step 7: letting $\delta \rightarrow 0$ in (78). We multiply the two equations of (78) by $\bar{w}_{\delta}$, and integrate over $\bar{D}_{-}$and, respectively, $\bar{D}_{+}$. This gives

$$
\int_{\bar{D}_{-}}\left(\bar{a}_{-} \bar{\nabla} \bar{w}_{\delta}\right) \bar{\nabla}_{w_{\delta}}+\delta \int_{\bar{D}_{-}}\left|\nabla \bar{w}_{\delta}\right|^{2}+\delta \int_{\bar{D}_{-}}\left(\bar{w}_{\delta}\right)^{2}=\int_{\Gamma}\left[\left(a_{-} \bar{\nabla}_{\delta}\right) \cdot e_{1}+\delta\left(\nabla \bar{w}_{\delta}\right) \cdot e_{1}\right] \bar{w}_{\delta},
$$

and

$$
\int_{\bar{D}_{+}}\left(\bar{a}_{+} \bar{\nabla} \bar{w}_{\delta}\right) \bar{\nabla} \bar{w}_{\delta}+\delta \int_{\bar{D}_{+}}\left|\nabla \bar{w}_{\delta}\right|^{2}+\delta \int_{\bar{D}_{+}}\left(\bar{w}_{\delta}\right)^{2}=-\int_{\Gamma}\left[\left(a_{+} \bar{\nabla} \bar{w}_{\delta}\right) \cdot e_{1}+\delta\left(\nabla \bar{w}_{\delta}\right) \cdot e_{1}\right] \bar{w}_{\delta},
$$

Summing up these equalities, and using the coupling condition across the interface in (78), we have

$$
\begin{aligned}
\int_{\bar{D}_{-}}\left(\bar{a}_{-} \bar{\nabla} \bar{w}_{\delta}\right) \bar{\nabla}_{w_{\delta}}+\delta \int_{\bar{D}_{-}}\left|\nabla \bar{w}_{\delta}\right|^{2}+\delta \int_{\bar{D}_{-}}\left(\bar{w}_{\delta}\right)^{2}+\int_{\bar{D}_{+}}\left(\bar{a}_{+} \bar{\nabla}_{\bar{w}}\right) \bar{\nabla}_{\bar{w}_{\delta}}+\delta \int_{\bar{D}_{+}}\left|\nabla \bar{w}_{\delta}\right|^{2}+\delta \int_{\bar{D}_{+}}\left(\bar{w}_{\delta}\right)^{2} \\
=\int_{\Gamma} \bar{\eta} \bar{w}_{\delta} .
\end{aligned}
$$

Using the definition $(72)$ of $\bar{\eta}$, we have

$$
\int_{\Gamma} \bar{\eta} \bar{w}_{\delta}=\int_{\Gamma}\left[\left(\bar{a}_{+}\left(\bar{\nabla} w_{+}+p\right)-a_{+}^{*} p\right) \cdot e_{1}\right] \bar{w}_{\delta}-\int_{\Gamma}\left[\left(\bar{a}_{-}\left(\bar{\nabla} w_{-}+p\right)-a_{-}^{*} p\right) \cdot e_{1}\right] \bar{w}_{\delta},
$$

where we recall that $w_{ \pm}$are solution to (45) and (46), respectively. In (95), we make a slight abuse of notation: $w_{ \pm}$is extended to $\mathbb{R}^{2 d-1}$ as follows:

$$
w_{-}\left(x_{1}, x_{2}, \ldots, x_{d}, y_{2}, \ldots, y_{d}\right):=w_{-}\left(x_{1}, x_{2}, \ldots, x_{d}\right),
$$


and

$$
w_{+}\left(x_{1}, x_{2}, \ldots, x_{d}, y_{2}, \ldots, y_{d}\right):=w_{+}\left(x_{1}, y_{2}, \ldots, y_{d}\right) .
$$

We now observe that, in the first term of the right-hand side $(95)$, the factor $\left(\bar{a}_{+}\left(\bar{\nabla} w_{+}+p\right)-a_{+}^{*} p\right) \cdot e_{1}$ does not depend on $x^{\prime}=\left(x_{2}, \ldots, x_{d}\right)$. Similarly, in the second term, the factor $\left(\bar{a}_{-}\left(\bar{\nabla} w_{-}+p\right)-a_{-}^{*} p\right) \cdot e_{1}$ does not depend on $y^{\prime}=\left(y_{2}, \ldots, y_{d}\right)$. Moreover, an application of Lemma 5.2 to each of these terms, shows that their integral over $\Gamma$ vanishes. Hence, setting

$$
\left\langle\bar{w}_{\delta}\right\rangle_{\Gamma}=\frac{1}{|\Gamma|} \int_{\Gamma} \bar{w}_{\delta}
$$

we have

$$
\begin{aligned}
& \int_{\Gamma} \bar{\eta} \bar{w}_{\delta} \\
& \quad=\int_{\Gamma}\left[\left(\bar{a}_{+}\left(\bar{\nabla} w_{+}+p\right)-a_{+}^{*} p\right) \cdot e_{1}\right]\left(\bar{w}_{\delta}-\left\langle\bar{w}_{\delta}\right\rangle_{\Gamma}\right)-\int_{\Gamma}\left[\left(\bar{a}_{-}\left(\bar{\nabla} w_{-}+p\right)-a_{-}^{*} p\right) \cdot e_{1}\right]\left(\bar{w}_{\delta}-\left\langle\bar{w}_{\delta}\right\rangle_{\Gamma}\right),
\end{aligned}
$$

Next, we are going to use the fact that in the integrands, some functions depend only on $x^{\prime}=\left(x_{2}, \ldots, x_{d}\right)$, and some depend only on $y^{\prime}=\left(y_{2}, \ldots, y_{d}\right)$. For this purpose, we set

$$
\Gamma=\{0\} \times \underbrace{\left[0, R_{2}\right] \times \cdots \times\left[0, R_{d}\right]}_{:=\Gamma_{x}} \times \underbrace{\left[0, S_{2}\right] \times \cdots \times\left[0, S_{d}\right]}_{:=\Gamma_{y}},
$$

We thus have

$$
\int_{\Gamma}\left[\left(\bar{a}_{+}\left(\bar{\nabla} w_{+}+p\right)-a_{+}^{*} p\right) \cdot e_{1}\right]\left(\bar{w}_{\delta}-\left\langle\bar{w}_{\delta}\right\rangle_{\Gamma}\right)=\int_{\Gamma_{y}}\left[\left(\bar{a}_{+}\left(\bar{\nabla} w_{+}+p\right)-a_{+}^{*} p\right) \cdot e_{1}\right]\left(\int_{\Gamma_{x}}\left(\bar{w}_{\delta}-\left\langle\bar{w}_{\delta}\right\rangle_{\Gamma}\right)\right)
$$

Setting

$$
W_{\delta}(y)=\int_{\Gamma_{x}}\left(\bar{w}_{\delta}-\left\langle\bar{w}_{\delta}\right\rangle_{\Gamma}\right)
$$

it is clear that $W_{\delta}$ has zero mean value over $\Gamma_{x}$. Hence, a combination of Cauchy-Schwarz inequality and a trace inequality gives

$$
\left|\int_{\Gamma}\left[\left(\bar{a}_{+}\left(\bar{\nabla} w_{+}+p\right)-a_{+}^{*} p\right) \cdot e_{1}\right]\left(\bar{w}_{\delta}-\left\langle\bar{w}_{\delta}\right\rangle_{\Gamma}\right)\right| \leq C\left(\int_{[0,1] \times \Gamma_{y}}\left(\left|\nabla_{y^{\prime}} W_{\delta}\right|^{2}+\left(\partial_{x_{1}} W_{\delta}\right)^{2}\right) d x_{1} d y^{\prime}\right)^{1 / 2}
$$

Now, we have

$$
\begin{aligned}
& \int_{[0,1] \times \Gamma_{y}}\left(\left|\nabla_{y^{\prime}} W_{\delta}\right|^{2}+\left(\partial_{x_{1}} W_{\delta}\right)^{2}\right) d y \\
= & \int_{[0,1] \times \Gamma_{y}}\left|\int_{\Gamma_{x}} \nabla_{y^{\prime}} \bar{w}_{\delta}\left(x_{1}, x^{\prime}, y^{\prime}\right) d x^{\prime}\right|^{2} d y^{\prime}+\int_{[0,1] \times \Gamma_{y}}\left|\int_{\Gamma_{x}} \partial_{x_{1}} \bar{w}_{\delta}\left(x_{1}, x^{\prime}, y^{\prime}\right) d x^{\prime}\right|^{2} d y^{\prime} \\
= & \int_{[0,1] \times \Gamma_{y}}\left|\int_{\Gamma_{x}} \bar{\nabla} \bar{w}_{\delta}\left(x_{1}, x^{\prime}, y^{\prime}\right) d x^{\prime}\right|^{2} d y^{\prime}+\int_{[0,1] \times \Gamma_{y}}\left|\int_{\Gamma_{x}} \partial_{x_{1}} \bar{w}_{\delta}\left(x_{1}, x^{\prime}, y^{\prime}\right) d x^{\prime}\right|^{2} d y^{\prime} \leq C \int_{\bar{D} \cap\left\{0<x_{1}<1\right\}}\left|\bar{\nabla} \bar{w}_{\delta}\right|^{2} .
\end{aligned}
$$

Here, we have used the fact that $\bar{w}_{\delta}$ is periodic with respect to $x$ in order to introduce an additional gradient term under the integral $\int_{\Gamma_{x}}$. We insert this estimate into (97), and use a similar argument to deal with the last term of (96). This finally gives

$$
\left|\int_{\Gamma} \overline{\eta w}_{\delta}\right| \leq\left(\int_{\bar{D}}\left|\bar{\nabla} \bar{w}_{\delta}\right|^{2}\right)^{1 / 2}
$$


We insert this estimate into (94), and find

$$
\begin{array}{r}
\underline{\mu} \int_{\bar{D}_{-}}\left|\bar{\nabla} \bar{w}_{\delta}\right|^{2}+\underline{\mu} \int_{\bar{D}_{+}}\left|\bar{\nabla} \bar{w}_{\delta}\right|^{2}+\delta\left(\left\|\bar{w}_{\delta}\right\|_{H^{1}\left(\bar{D}_{-}\right)}^{2}+\left\|\bar{w}_{\delta}\right\|_{H^{1}\left(\bar{D}_{+}\right)}^{2}\right) \\
\leq C\left(\left\|\bar{\nabla} \bar{w}_{\delta}\right\|_{L^{2}\left(\bar{D}_{-}\right)}+\left\|\bar{\nabla} \bar{w}_{\delta}\right\|_{L^{2}\left(\bar{D}_{+}\right)}\right) .
\end{array}
$$

Here, we have used the fact that $a$ is elliptic. Consequently, we have the following bounds:

$$
\begin{gathered}
\left\|\bar{\nabla} \bar{w}_{\delta}\right\|_{L^{2}\left(\bar{D}_{-}\right)}+\left\|\bar{\nabla}_{\bar{w}_{\delta}}\right\|_{L^{2}\left(\bar{D}_{+}\right)} \leq C, \\
\left\|\bar{w}_{\delta}\right\|_{H^{1}\left(\bar{D}_{-}\right)}+\left\|\bar{w}_{\delta}\right\|_{H^{1}\left(\bar{D}_{+}\right)} \leq \frac{C}{\sqrt{\delta}}
\end{gathered}
$$

both for a constant $C$ independent of $\delta>0$. Now, estimate (100) allows to extract a subsequence such that $\bar{\nabla} \bar{w}_{\delta}$ converges weakly to some limit $V$. This limit is a partial gradient due to the fact that differential operators are continuous with respect to weak convergence topology: $V=\bar{\nabla} w_{0}$. Moreover, (101) allows to pass to the limit $\delta \rightarrow 0$ in the weak formulation of (78). We thus have a solution to (75).

Step 8: taking the trace $x^{\prime}=y^{\prime}$. We now need to conclude the existence part of Theorem 5.7, proving that we may obtain a solution to (71) from that of (75). For this purpose, as indicated in Step 2, we need regularity, since we need to take the trace $x^{\prime}=y^{\prime}$. The passage to the limit we performed in Step 7 is not sufficient to get that regularity. In order to indeed obtain that regularity, we make the following observation (which crucially exploits the regularity of the coefficient $a$ of our equation). We note that we may differentiate the equations with respect to $x^{\prime}$. Setting $u=\partial_{x^{\prime}} \bar{w}_{\delta},(78)$ yields

$$
\left\{\begin{array}{l}
-\overline{\operatorname{div}}\left(\bar{a}_{-} \bar{\nabla} u_{-}\right)-\delta \Delta u_{-}+\delta u_{-}=\overline{\operatorname{div}}\left(\partial_{x^{\prime}} \bar{a}_{-} \bar{\nabla} \bar{w}_{\delta,-}\right) \text { in }\left\{x_{1}<0\right\} \\
\bar{\nabla} u_{-} \in L^{2}\left(\bar{D}_{-}\right), \\
-\overline{\operatorname{div}}\left(\bar{a}_{+} \bar{\nabla} u_{+}\right)-\delta \Delta u_{+}+\delta u_{+}=\overline{\operatorname{div}}\left(\partial_{x^{\prime}} \bar{a}_{+} \bar{\nabla} \bar{w}_{\delta,+}\right) \text { in }\left\{x_{1}>0\right\} \\
\bar{\nabla} u_{+} \in L^{2}\left(\bar{D}_{+}\right), \\
\left(\bar{a}_{+} \bar{\nabla} u_{+}\right) \cdot e_{1}+\delta \nabla u_{+} \cdot e_{1}=\left(\bar{a}_{-} \bar{\nabla} u_{-}\right) \cdot e_{1}+\delta \nabla u_{-} \cdot e_{1}+\partial_{x^{\prime}} \bar{\eta} \\
\quad \text { on }\left\{x_{1}=0\right\} \\
u_{ \pm} \text {is periodic in } x_{2}, \ldots, x_{d}, y_{2}, \ldots, y_{d}
\end{array}\right.
$$

Hence, we may repeat our argument of Step 7 for $u$ instead of $\bar{w}_{\delta}$. This leads to an estimate similar to (99), with an additional term due to the right-hand side of (102), namely:

$$
\begin{aligned}
& \underline{\mu} \int_{\bar{D}_{-}}|\bar{\nabla} u|^{2}+\underline{\mu} \int_{\bar{D}_{+}}|\bar{\nabla} u|^{2}+\delta\left(\|u\|_{H^{1}\left(\bar{D}_{-}\right)}^{2}+\|u\|_{H^{1}\left(\bar{D}_{+}\right)}^{2}\right) \\
& \quad \leq C\left(\|\bar{\nabla} u\|_{L^{2}\left(\bar{D}_{-}\right)}+\|\bar{\nabla} u\|_{L^{2}\left(\bar{D}_{+}\right)}\right)+C\left(\|\bar{\nabla} u\|_{L^{2}\left(\bar{D}_{-}\right)}\left\|\bar{\nabla} \bar{w}_{\delta}\right\|_{L^{2}\left(\bar{D}_{-}\right)}+\|\bar{\nabla} u\|_{L^{2}\left(\bar{D}_{+}\right)}\left\|\bar{\nabla} \bar{w}_{\delta}\right\|_{L^{2}\left(\bar{D}_{+}\right)}\right) .
\end{aligned}
$$

Hence, inserting (100) into the last line of (103), we find that $u=\partial_{x^{\prime}} \bar{w}_{\delta}$ satisfies (100) and (101). Iterating this argument, one easily shows that any derivative of $\bar{w}_{\delta}$ with respect to $x^{\prime}$ satisfies (100) and (101). The same property is true for derivatives with respect to $y^{\prime}$. Hence, we see that $\left\|\bar{\nabla} \nabla^{s} \bar{w}_{\delta}\right\|_{L^{2}\left(D_{ \pm}\right)}$is bounded independently of $\delta$, for any $s \geq 0$. Hence, taking $s$ sufficiently large, the limit is continuous. We can take its trace on the set $x^{\prime}=y^{\prime}$, thus finding a solution to (71). Indeed, the first and third line of (71) are clear. The second and fourth line are a simple consequence of the fact that $\bar{\nabla} \bar{w}_{\delta}$ is periodic in $x^{\prime}, y^{\prime}$ and bounded in $L^{2}\left(\bar{D}_{ \pm}\right)$. Finally, we note that the solution we obtain is quasiperiodic with respect to $x^{\prime}$, and that its gradient has mean value zero. Hence this solution is sublinear at infinity with respect to $x^{\prime}$ (that is, in the directions parallel to the interface). 
Step 9: uniqueness. Consider two solutions of (65). Their difference, which we denote by $u$, satisfies $-\operatorname{div}(a \nabla u)=0$ in $\mathbb{R}^{d}$. In addition, $\nabla u$ is quasiperiodic in $x^{\prime}=\left(x_{2}, \ldots, x_{d}\right)$, and satisfies

$$
\int_{\mathbb{R}}\left\langle|\nabla u|^{2}\right\rangle_{\mathbb{R}^{d-1}} d x_{1}<+\infty
$$

where $\langle\cdot\rangle_{\mathbb{R}^{d-1}}$ denotes the average with respect to the variable $x^{\prime}$. Hence, we may apply Lemma A.2: there exists a sequence of smooth functions $\left(u_{k}\right)_{n \in \mathbb{N}}$ having compact support in $x_{1}$ such that

$$
\int_{\mathbb{R}}\left\langle\left|\nabla u_{k}-\nabla u\right|^{2}\right\rangle_{\mathbb{R}^{d-1}} d x_{1} \underset{n \rightarrow+\infty}{\longrightarrow} 0 .
$$

We multiply the equation $-\operatorname{div}(a \nabla u)=0$ by $u_{k}$, integrate it over the domain $B_{R}^{\prime}=\left\{x \in \mathbb{R}^{d}, \quad\left|x^{\prime}\right| \leq R\right\}$, and integrate by parts:

$$
\int_{B_{R}^{\prime}}(a \nabla u) \cdot \nabla u_{k}=\int_{\partial B_{R}^{\prime}} u_{k}(a \nabla u) \cdot n \text {. }
$$

Hence,

$$
\left|\frac{1}{R^{d-1}} \int_{B_{R}^{\prime}}(a \nabla u) \cdot \nabla u_{k}\right| \leq\|\nabla u\|_{L^{\infty}}\left\|u_{k}\right\|_{L^{\infty}} \frac{R^{d-2}}{R^{d-1}} \underset{R \rightarrow+\infty}{\longrightarrow} 0 .
$$

We conclude that, for any $k \in \mathbb{N}$,

$$
\int_{\mathbb{R}}\left\langle(a \nabla u) \cdot \nabla u_{k}\right\rangle_{\mathbb{R}^{d-1}} d x_{1}=0
$$

Letting $k \rightarrow+\infty$, and using (104), we infer

$$
\int_{\mathbb{R}}\left\langle|\nabla u|^{2}\right\rangle_{\mathbb{R}^{d-1}} d x_{1}=0
$$

hence, $\nabla u=0$.

Step 10: the case of non-Liouville-Roth numbers. Here, we deal with the case where (68) is satisfied. All the above steps are still valid. Step 8 implies that the solution $\bar{w}$ satisfies $\bar{\nabla} \bar{w} \in H^{s}\left(\bar{D}_{ \pm}\right)$, for any $s \geq 0$. Hence, applying Lemma 5.5, we have

$$
\bar{w}-\langle\bar{w}\rangle \in L^{2}\left(\bar{D}_{ \pm}\right),
$$

where, as above, $\langle\bar{w}\rangle=\langle\bar{w}\rangle\left(x_{1}\right)$ is the average of $\bar{w}$ with respect to variables $x^{\prime}, y^{\prime}$. In fact, we may also apply Lemma 5.5 to any derivative of $\bar{w}$, so that

$$
\bar{w}-\langle\bar{w}\rangle \in H^{s}\left(\bar{D}_{ \pm}\right), \quad \forall s \geq 0 .
$$

As a consequence, $\bar{w}-\langle\bar{w}\rangle \in C^{0} \cap L^{\infty}\left(\mathbb{R}^{2 d+1}\right)$. Taking the trace, we thus have that $\tilde{w}-\langle\bar{w}\rangle\left(x_{1}\right) \in L^{\infty}\left(\mathbb{R}^{d}\right)$, where

$$
\forall x_{1} \in \mathbb{R}, \quad \forall x^{\prime} \in \mathbb{R}^{d-1}, \quad \tilde{w}\left(x_{1}, x^{\prime}\right)=\bar{w}\left(x_{1}, x^{\prime}, x^{\prime}\right) .
$$

We now fix $x_{1} \in \mathbb{R}$, and infer that $x^{\prime} \mapsto \widetilde{w}\left(x_{1}, x^{\prime}\right)$ is bounded, and that its gradient is quasiperiodic. Hence, applying Lemma 5.6, we have that $\widetilde{w}$ is quasiperiodic. We also note that, due to its definition, we have

$$
\forall x_{1} \in \mathbb{R}, \quad\langle\tilde{w}\rangle\left(x_{1}\right)=\langle\bar{w}\rangle\left(x_{1}\right),
$$

where the first notation $\langle\cdot\rangle$ denotes the mean value of quasiperiodic functions, and the second one stands for mean value of periodic functions.

It remains to prove that $\tilde{w}$ is bounded with respect to $x_{1}$. We give the proof for $x_{1}>0$, the same argument being valid for $x_{1}<0$. For this purpose, we first recall that,

$$
\forall s \geq 1, \quad\left\langle\left|\nabla^{s} \tilde{w}\right|^{2}\right\rangle \in L^{1}\left(\mathbb{R}^{+}\right) .
$$

Hence, defining

$$
F_{s}\left(x_{1}\right)=\left\langle\left|\nabla^{s} \tilde{w}\right|^{2}\right\rangle\left(x_{1}\right)
$$


for $s \geq 1$, and

$$
F_{0}\left(x_{1}\right)=\left\langle|\tilde{w}-\langle\tilde{w}\rangle|^{2}\right\rangle\left(x_{1}\right)
$$

we have $F_{s} \in L^{1}\left(\mathbb{R}^{+}\right)$, for any $s \geq 0$. In addition,

$$
\forall s \geq 1, \quad\left|F_{s}^{\prime}\left(x_{1}\right)\right| \leq\left\langle 2\left|\nabla^{s} \partial_{x_{1}} \tilde{w}\right|\left|\nabla^{s} \tilde{w}\right|\right\rangle \leq 2 \sqrt{F_{s+1}\left(x_{1}\right)} \sqrt{F_{s}\left(x_{1}\right)} \in L^{1}\left(\mathbb{R}^{+}\right),
$$

and

$$
\left|F_{0}^{\prime}\left(x_{1}\right)\right| \leq 2\left\langle\left|\partial_{x_{1}}(\tilde{w}-\langle\tilde{w}\rangle)\right||\tilde{w}-\langle\tilde{w}\rangle|\right\rangle \leq 2 \sqrt{F_{1}\left(x_{1}\right)} \sqrt{F_{0}\left(x_{1}\right)} \in L^{1}\left(\mathbb{R}^{+}\right) .
$$

Similarly, for any integer $q \geq 0, F_{s}^{(q)} \in L^{1}\left(\mathbb{R}^{+}\right)$. This also implies that $F_{s}^{(q)} \in L^{\infty}\left(\mathbb{R}^{+}\right)$.Hence,

$$
\forall s \geq 0, \quad \forall q \geq 0, \quad F_{s}^{(q)} \in L^{1}\left(\mathbb{R}^{+}\right) \cap L^{\infty}\left(\mathbb{R}^{+}\right) .
$$

Next, we define

$$
G_{s}(T)=\int_{T}^{+\infty} F_{s}\left(x_{1}\right) d x_{1} .
$$

Multiplying the equation $\operatorname{div}(a \nabla \tilde{w})=0\left(\right.$ if $\left.x_{1} \neq 0\right)$ by $\tilde{w}$, and integrating over the domain $\left\{\left|x^{\prime}\right| \leq R, T \leq x_{1} \leq T^{\prime}\right\}$, where $T>0$, one gets

$$
\begin{aligned}
\int_{\left\{\left|x^{\prime}\right| \leq R, T \leq x_{1} \leq T^{\prime}\right\}}(a \nabla \tilde{w}) \cdot \nabla \tilde{w}=\int_{\left\{\left|x^{\prime}\right|=R,\right.} & \left.T \leq x_{1} \leq T^{\prime}\right\} \\
& +\int_{\left\{\left|x^{\prime}\right| \leq R, x_{1}=T^{\prime}\right\}} \tilde{w}(a \nabla \tilde{w}) \cdot n
\end{aligned}
$$

Dividing by $R^{d-1}$, using the fact that all functions are quasiperiodic in $x^{\prime}$, letting $R \rightarrow+\infty$, and using that $a$ is elliptic, we infer

$$
\underline{\mu} \int_{T}^{T^{\prime}}\left\langle|\nabla \tilde{w}|^{2}\right\rangle \leq\left\langle\tilde{w}(a \nabla \tilde{w}) \cdot e_{1}\right\rangle\left(T^{\prime}\right)-\left\langle\tilde{w}(a \nabla \tilde{w}) \cdot e_{1}\right\rangle(T) .
$$

We integrate the equation over the same domain and use the same strategy to prove that

$$
\forall T, T^{\prime}>0, \quad\left\langle(a \nabla \tilde{w}) \cdot e_{1}\right\rangle\left(T^{\prime}\right)=\left\langle(a \nabla \tilde{w}) \cdot e_{1}\right\rangle(T) .
$$

Hence, $T \mapsto\left\langle(a \nabla \tilde{w}) \cdot e_{1}\right\rangle(T)$ is a constant. If this constant is not 0 , then we reach a contradiction with the fact that $\left\langle|\nabla \tilde{w}|^{2}\right\rangle \in L^{1}\left(\mathbb{R}^{+}\right)$. Hence, we have the equivalent of (62) in the present quasiperiodic setting. Using this in (106), we see that we can add any constant to $\tilde{w}$ in each term of the right-hand side. Hence,

$$
\int_{T}^{T^{\prime}} F_{1}\left(x_{1}\right) d x_{1} \leq C \sqrt{F_{1}\left(T^{\prime}\right) F_{0}\left(T^{\prime}\right)}+C \sqrt{F_{1}(T) F_{0}(T)},
$$

for some constant $C$ independent of $T$ and $T^{\prime}$. Using the fact that $F_{0}, F_{1} \in L^{1}$, we can find a sequence $T^{\prime} \rightarrow+\infty$ such that the first term of the right-hand side vanishes. Hence,

$$
G_{1}(T)=\int_{T}^{+\infty} F_{1}\left(x_{1}\right) d x_{1} \leq \sqrt{F_{1}(T) F_{0}(T)} \leq C \sqrt{F_{1}(T)}=C \sqrt{-G_{1}^{\prime}(T)} .
$$

Here, we have used the bound (105). Applying Lemma A.3 to $G_{1}$, we thus have

$$
\int_{T}^{+\infty} F_{1}\left(x_{1}\right) d x_{1} \leq \frac{C_{1}}{T}
$$

for some constant $C_{1}$ independent of $T$. We can repeat the same argument with $F_{s}$ instead of $F_{1}$, for any $s \geq 1$. Indeed, as pointed out above, $\nabla^{s} \tilde{w}$ satisfies the same equation. The only difference is that the right-hand side is not zero, introducing an additional term, giving, instead of (107):

$$
G_{s}(T)=\int_{T}^{+\infty} F_{s}\left(x_{1}\right) d x_{1} \leq C \sqrt{F_{s}(T) F_{s-1}(T)}+C \int_{T}^{+\infty} \sqrt{F_{s-1}\left(x_{1}\right) F_{s}\left(x_{1}\right)} d x_{1} .
$$


Hence, we can argue by induction: we have (108), that is,

$$
\int_{T}^{+\infty} F_{s}\left(x_{1}\right) d x_{1} \leq \frac{C_{s}}{T}
$$

for $s=1$. If it holds for $F_{s-1}$, then we have, applying $(105), G_{s}(T) \leq C \sqrt{F_{s}(T)}+\frac{1}{2} G_{s}(T)+C G_{s-1}(T)$, that is,

$$
G_{s}(T) \leq C \sqrt{-G_{s}^{\prime}(T)}+\frac{C}{T}
$$

Consequently, by application of Lemma A.3, we get

$$
G_{s}(T)=\int_{T}^{+\infty} F_{s}\left(x_{1}\right) d x_{1} \leq \frac{C_{s}}{T},
$$

where $C_{s}$ depends on $s$ but not on $T$. Then, we have

$$
F_{s}(T) \leq \int_{T}^{+\infty}\left|F_{s}^{\prime}\left(x_{1}\right)\right| d x_{1} \leq 2\left(\int_{T}^{+\infty} F_{s+1}\right)^{1 / 2}\left(\int_{T}^{+\infty} F_{s}\right)^{1 / 2}
$$

Thus, (109) implies that, for any $s \geq 1$,

$$
F_{s}(T) \leq \frac{C}{T}
$$

Applying Lemma 5.5 again, we prove that (110) is also valid for $s=0$. Hence, going back to (107), we infer

$$
G_{1}(T)=\int_{T}^{+\infty} F_{1}\left(x_{1}\right) d x_{1} \leq \frac{C}{\sqrt{T}} \sqrt{F_{1}(T)}=\frac{C}{\sqrt{T}} \sqrt{-G_{1}^{\prime}(T)} .
$$

Dividing this inequality by $G_{1}(T)$, we find $-\frac{G_{1}^{\prime}}{G_{1}^{2}} \geq C T$, hence, integrating with respect to $T$,

$$
\int_{T}^{+\infty} F_{1}\left(x_{1}\right) d x_{1}=G_{1}(T) \leq \frac{C}{T^{2}} .
$$

Then, we define $\varphi=\langle\tilde{w}\rangle$. It clearly satisfies $\varphi^{\prime}\left(x_{1}\right)=\left\langle\partial_{x_{1}} \tilde{w}\right\rangle$, hence $\left|\varphi^{\prime}\left(x_{1}\right)\right| \leq F_{1}\left(x_{1}\right)$. We then apply Lemma A.4 to $\varphi$, which proves that $x_{1} \mapsto\langle\tilde{w}\rangle\left(x_{1}\right)$ is bounded. Using the fact that $F_{0} \in L^{\infty}\left(\mathbb{R}^{+}\right)$and that $\tilde{w}$ is smooth, we conclude that $\tilde{w}$ is bounded.

Remark 22 As briefly mentioned above, the Schwarz iteration technique employed in Steps 4 through 6 to prove that the problem (78) has a solution is only one option. An alternative proof is based upon the same argument as that used in Section 5.2 in the proof of Theorem 5.1 since the problem is periodic in the directions transverse to $x_{1}$ and is elliptic.

Remark 23 In the spirit of Remark 17, we may observe that, when the two periodic coefficients $a_{-}$and $a_{+}$ on the two sides of the interface are close to one another (say in $L^{\infty}$ norm), then it is possible to prove a result similar to Theorems 5.1 and 5.7 using an argument based on the Banach fixed point Theorem. In the setting of Section 5.2 and Theorem 5.1, it is a simple application of the proof described in Remark 17. In the setting of Theorem 5.7, the fixed point argument may be used to circumvent the proof of Steps 4 through 6 on the regularized problem (78), while the other steps of the proof remain unchanged.

Remark 24 We have used above the fact that if $\bar{w}$ is a solution of the problem in the augmented dimension space (75), then its trace is a solution to the original problem (71). Actually, we can prove that these two problems are equivalent. The main difficulty here is that $w$ itself is a priori not quasiperiodic. If it were, the result would be easy to prove. 
Indeed, consider a solution $w$ to the original problem (71), which gradient is quasiperiodic. Then, we know that $\nabla w$ is quasiperiodic. Hence, there exists $W: \mathbb{R}^{2 d-1} \rightarrow \mathbb{R}^{d}$ which is periodic, and such that $\nabla w(x)=W(x, x)$. As a consequence, we have

$$
\forall i, j \geq 2, \quad\left(\frac{\partial_{x_{i}+y_{i}}}{2} W_{j}\right)(x, x)=\left(\partial_{\frac{x_{j}+y_{j}}{2}} W_{i}\right)(x, x),
$$

and

$$
\forall i \geq 2, \quad\left(\partial_{\frac{x_{i}+y_{i}}{2}} W_{1}\right)(x, x)=\left(\partial_{x_{1}} W_{i}\right)(x, x)
$$

Hence, there exists a function $\bar{w}: \mathbb{R}^{2 d-1} \rightarrow \mathbb{R}$ such that $W=\bar{\nabla} \bar{w}$. Now, we have

$$
\left.\overline{\operatorname{div}}(\bar{a} \bar{\nabla} \bar{w})\right|_{x=y}=\operatorname{div}(a \nabla w) .
$$

Not that here, we use the fact that $\bar{a}$ is smooth. Hence, the equation in the augmented dimension space is satisfied by $\bar{w}$ in the set $\{x=y\}$. Now, due to the fact that we deal with periodic functions with incommensurable periods, the equation is satisfied in a set which is dense in the unit cell. Hence, by continuity of the functions involved, it is satisfied everywhere.

We conclude this section with some considerations on a problem of a similar nature to that examined here: the corrector problem for quasiperiodic homogenization.

\subsection{Remarks on the corrector in quasiperiodic homogenization}

Consider a quasiperiodic coefficient $a$, which enjoys all the usual properties necessary to correctly consider the oscillatory elliptic equation (1). The question of determining the homogenized limit of that equation is classical. The traditional approach to that problem is to embed the quasiperiodic setting into the almost periodic setting, and to interpret the latter as a particular case of a random stationary ergodic setting:

$$
-\operatorname{div}\left(a(x / \varepsilon, \omega) \nabla u^{\varepsilon}(x, \omega)\right)=f
$$

using as abstract probability space the Bohr compactification and as abstract probability measure the Haar measure. Doing so, one obtains the existence of a homogenized limit for (111). In passing, one establishes the existence of a corrector, in the sense that the equation

$$
\left\{\begin{array}{l}
-\operatorname{div}\left(a(., \omega)\left(p+\nabla w_{p}(., \omega)\right)\right)=0 \\
\frac{w_{p}(x, \omega)}{1+|x|} \stackrel{|x| \rightarrow \infty}{\longrightarrow} 0
\end{array}\right.
$$

(analogous to (8)) is shown to almost surely have a solution. Put differently, the existence of a corrector is known almost surely. Now, even though $\mathbb{R}^{d}$ is included in its Bohr compactification, we cannot infer any information from (112) regarding a quasiperiodic corrector. Indeed, $\mathbb{R}^{d}$ is of zero measure in that space. Otherwise, the restriction of the Haar measure to $\mathbb{R}^{d}$, being translation invariant, would be a multiple of the Lebesgue measure, which cannot be of finite mass (see [1, Theorem 2.7] for a detailed proof). In addition, the corrector provided by this stochastic setting is only known to have a gradient that is stationary and of zero mean, that is, in the present context, to be an almost periodic gradient with zero mean. Although this information is sufficient to proceed with the theory of homogenization for the equation (111), it is a fair question to ask whether, in the specific context of a quasiperiodic setting, one can obtain a better information, that is: (i) solve the corrector equation (and not "almost surely solve it") and (ii) determine whether the corrector, or its gradient, has quasiperiodic (and not only almost periodic) properties. Similar delicate questions have been excellently addressed in [1].

When the quasiperiodic coefficient is sufficiently regular, it turns out that the ingredients of the above proof of Theorem 5.7 allow to completely clarify the situation and answer to the above two questions, as was announced and outlined in [28]. We have the following result: 
Theorem 5.8 Consider

$$
\left\{\begin{array}{l}
-\operatorname{div}\left(a\left(p+\nabla w_{p}\right)\right)=0 \\
\frac{w_{p}(x)}{1+|x|} \stackrel{|x| \rightarrow \infty}{\longrightarrow} 0
\end{array}\right.
$$

for a quasiperiodic coefficient a enjoying the usual coercivity and continuity assumptions. Assume (for simplicity) that the periodic coefficient $\bar{a}$, the trace of which is a, is smooth, has periods $R_{i}, S_{i}, 1 \leq i \leq d$, which, for simplicity of exposition again, we assume non commensurable: $R_{i} / S_{i} \notin \mathbb{Q}$, for all $1 \leq i \leq d$. Then there exists a solution $w_{p}$ to (113). The solution is unique up to the addition of a constant, and smooth. The gradient $\nabla w_{p}$ of that function is quasiperiodic, has zero mean, is bounded with all derivatives bounded. If, in addition, the quasiperiods of a give rise to quotients $R_{i} / S_{i}$ that all are not Liouville-Roth numbers, then $w_{p}$ itself is a quasiperiodic, bounded function.

Remark 25 As briefly mentioned above, Kozlov increased the dimension in [25] in order to transform a quasiperiodic problem into a periodic problem, and study homogenization beyond the periodic setting without explicitly resorting to the random stationary setting. The specifics of the proof in [25] are different (a Galerkin approximation technique is employed, instead of a regularization as we do here) but the pattern of the proof is definitely similar, and the proof also makes use a Gärding type inequality (Lemma 5.5 above). We however wish to point out that, even though the ingredients (augmented dimension and Gärding type inequality) are identical, the purpose of the proof in [25] is not to establish the existence of a corrector in the quasiperiodic setting. That article proves the existence of a corrector with an almost-periodic gradient when the coefficient is a trigonometric polynomial, then argues by density to prove homogenization in the almost periodic case. Also, the quasiperiodic character and the boundedness of the corrector itself (as opposed to its gradient), depending on the Liouville-Roth nature of the quasiperiods, are not addressed in [25]. We finally notice that the paper [13] elaborates on the results of [25] to address some nonlinear problems.

Sketch of proof: One first lifts equation (112) in an equation in a space of higher dimension, as in Step 2 of the proof of Theorem 5.7. That equation is not elliptic, and thus one regularizes it, as was done when passing from system (71) to system (78) in Step 3. It is easily shown that this regularized equation

$$
-\overline{\operatorname{div}}\left(a\left(\bar{\nabla}_{\bar{w}}+p\right)\right)-\delta \Delta \bar{w}_{\delta}+\delta \bar{w}_{\delta}=0,
$$

has a solution. Here, we use notation similar to those of the proof of Theorem 5.7, that is, $\bar{w}_{\delta}$ is the function in the higher dimensional space, $w_{\delta}$ being its trace, and $\overline{\operatorname{div}}$ and $\bar{\nabla}$ are degenerate differential operators defined as in (73) and (74). Thus, $\bar{w}_{\delta}$ is periodic, and a simple computation shows that

$$
\left\langle\left|\bar{\nabla} \bar{w}_{\delta}\right|^{2}\right\rangle+\delta\left\langle\left|\nabla \bar{w}_{\delta}\right|^{2}\right\rangle+\delta\left\langle\bar{w}_{\delta}^{2}\right\rangle \leq C
$$

for some constant $C$ that does not depend on $\delta$. Here, $\langle\cdot\rangle$ denotes the average of the periodic function considered. This allows to pass to the limit $\delta \rightarrow 0$, finding a solution to the equation with $\delta=0$. Moreover, $\bar{w}_{\delta}$ satisfies $\left\langle\bar{\nabla} \bar{w}_{\delta}\right\rangle=0$, which passes to the limit, hence $\langle\bar{\nabla} \bar{w}\rangle=0$. Then, Step 8 of the proof of Theorem 5.7 applies to the present case, proving that $\bar{\nabla} \bar{w}$ is smooth, so we can take its trace and find a solution to the original equation.

Next, we prove uniqueness. For this purpose, we consider the difference $u$ of two solutions, which is such that $\nabla u$ is quasiperiodic, has zero mean value, and satisfies

$$
-\operatorname{div}(a \nabla u)=0
$$

in $\mathbb{R}^{d}$. Hence, when integrating (115) against $u$ over the ball $B_{R}$, we find

$$
\frac{1}{\left|B_{R}\right|} \int_{B_{R}}|\nabla u|^{2} \leq \frac{C}{\left|B_{R}\right|} \int_{\partial B_{R}}|\nabla u||u| \text {. }
$$

We bound the right-hand side using the fact that $a$ is Hölder continuous. This implies that $\nabla u \in L^{\infty}$, hence

$$
\frac{1}{\left|B_{R}\right|} \int_{B_{R}}|\nabla u|^{2} \leq \frac{C}{\left|B_{R}\right|} \int_{\partial B_{R}}|u|
$$


Since $\langle\nabla u\rangle=0$, we know that $u$ is sublinear at infinity. Thus,

$$
\frac{1}{\left|B_{R}\right|} \int_{B_{R}}|\nabla u|^{2} \leq \frac{C}{\left|B_{R}\right|}\left|\partial B_{R}\right| o(R) \underset{R \rightarrow+\infty}{\longrightarrow} 0 .
$$

Hence, $|\nabla u|^{2}$ is a non-negative quasiperiodic function having zero average. It must be zero. Note that, if $u$ itself is quasiperiodic, it is bounded, and the above argument is not needed. Even if $a$ is not smooth, the above argument may be adapted by introducing a smooth cut-off function in (116).

Finally, we consider the case of non-Liouville-Roth numbers. Here again, we know that the solution $w_{\delta}$ we have built satisfies $\left\|\bar{w}_{\delta}\right\|_{H^{s}(Q)} \leq C$, for any $s \geq 0$, where $C$ does not depend on $\delta$. Here, $Q$ is the unit cell of the periodic lattice we use in the space of augmented dimension. We apply Lemma 5.5, which implies that $\bar{w}_{\delta}$ itself is bounded in $H^{s}(Q)$, for any $s \geq 0$. As a consequence, we may pass to the limit $\delta \rightarrow 0$, and find $\bar{w}=\lim \left(\bar{w}_{\delta}\right)$, which is smooth, bounded, periodic, and solution to (114). Hence, we can take its trace. It is a solution to (113), and is quasiperiodic and bounded.

Remark 26 As it was pointed out in Remark 24, the problem set in the augmented dimension space is equivalent to the one in the original space. The proof of this fact is an easy adaptation of that of Remark 24. Here again, the main difficulty is that $w$ itself is not quasiperiodic, otherwise the result would be simple.

Remark 27 In the above proof, uniqueness is proved in the original space $\mathbb{R}^{d}$. However, since the problem in the space of augmented dimension is equivalent to the original one, it is also possible to prove it in this space. Then, one deals with periodic functions, but with a degenerate elliptic operator. The spirit of the proof is the same: integrating the equation over a large domain, letting this domain go to infinity, and proving that the average of $|\nabla u|^{2}$ is zero.

Remark 28 The technique of proof of Theorem 5.8 carries over to the quasiperiodic homogenization of an equation that is not in divergence form, up to some necessary adaptations. We refer to [28] for an exposition of the results obtained.

Remark 29 Of course the cases when some of the $R_{i} / S_{i}$ are rational, or some of them are Liouville-Roth numbers and some others not, can be easily addressed, based on the result and techniques of proof of the above theorem. Likewise, if a is not smooth but has regularity $C^{k}$ for $k$ large enough, the result can be easily adapted.

Remark 30 Similar questions could be considered about almost periodic homogenization instead of quasiperiodic homogenization. Not everything seems clear to us in that setting yet (see [1] for a work in this vein): is it possible to prove existence of a corrector without using the general stochastic homogenization theory? And is it possible, in some specific cases to be determined, to prove that the corrector itself (not only its gradient) is almost periodic and/or bounded? As we already mentioned (see Remark 25), in [25], Kozlov used a similar strategy but did not study this question: he proved that homogenization holds without proving that the corrector exists.

\section{$6 \quad$ Related issues and future works}

We briefly approach in this section a topic we would like to more thoroughly explore in forthcoming works.

In the linear elliptic case examined in details in this article, we have seen that the presence of a local defect (modeled by the term $b$, say in $L^{2}\left(\mathbb{R}^{d}\right)$ for simplicity) does not modify the macroscopic behavior (that is, the homogenized matrix and the homogenized solution) but only affects the approximation of the exact solution $u^{\varepsilon}$ locally microscopically. It has also some macroscopic effects (see our Remark 5), but only in stronger Sobolev norms.

In order to show that a local defect may have more drastic consequences, even at the macroscopic scale, we consider the case of a Hamilton-Jacobi type equation. The insertions of "defects" for the homogenization of such equations will be studied in details in [29], but we wish to give here a flavor of the new difficulties and phenomena that arise in this setting. 
For this purpose, we consider the first-order Hamilton-Jacobi equation

$$
u^{\varepsilon}+\left|\nabla u^{\varepsilon}\right|=b(x / \varepsilon),
$$

posed on $\mathbb{R}^{d}$, with a right-hand side $b$ that satisfies

$$
b \in \mathcal{D}\left(\mathbb{R}^{d}\right), \quad b \leq 0, \quad \inf _{\mathbb{R}^{d}} b<0,
$$

and, to fix the ideas,

$$
b(0)=\inf _{\mathbb{R}^{d}} b .
$$

Intuitively, $b$ models a defect with respect to a periodic background $V_{\text {per }}(x)$ for the general Hamilton-Jacobi equation

$$
u^{\varepsilon}+\left|\nabla u^{\varepsilon}\right|=V_{p e r}(x / \varepsilon)+b(x / \varepsilon)
$$

and we have chosen the simplest possible case where $V_{\text {per }} \equiv 0$. In view of the results obtained in this article on the linear elliptic equation, one could (apparently) reasonably anticipate that, when $\varepsilon \rightarrow 0$, the solution $u^{\varepsilon}$ to (117) converges to that to

$$
u+|\nabla u|=0,
$$

which, when that equation is complemented with ad hoc boundary conditions "at infinity", is unique and is identically zero. Actually, we are going to see that this expected behavior is not correct. We mention that the essential ingredient responsible for the strange, unexpected behavior of $u^{\varepsilon}$ is the condition $\inf _{\mathbb{R}^{d}} b<0$ in (118) (all the other conditions are taken here for simplicity, and the behavior would be in line with the results of the linear case if $b$ were nonnegative). In the case of a fully nonlinear equation such as (117), the presence of the defect can significantly modify, at the macroscopic scale, the homogenized limit. In some loose sense, such a nonlinear equation is much less "forgiving" (that is, robust with respect to local perturbations) than a simple elliptic equation.

To start with, we make everything explicit and simple: we consider the one-dimensional version of (117)

$$
u^{\varepsilon}+\left|\left(u^{\varepsilon}\right)^{\prime}\right|=b(x / \varepsilon),
$$

set on the whole real line, with the condition that $u^{\varepsilon}$ vanishes at $\pm \infty$. Again to make things simple, we additionally assume that $b^{\prime}(x) \geq 0$ for $x>0$ and $b^{\prime}(x) \leq 0$ for $x<0$. Then it is immediate to see that the unique $C^{1}$ solution to (121) reads

$$
u^{\varepsilon}(x)= \begin{cases}b(0) e^{x}+e^{x} \int_{x}^{0} e^{-t} b(t / \varepsilon) d t, & \text { for } \quad x \leq 0, \\ b(0) e^{-x}+e^{-x} \int_{0}^{x} e^{t} b(t / \varepsilon) d t, & \text { for } \quad x \geq 0 .\end{cases}
$$

One then immediately reads the limit of $u^{\varepsilon}$ on this formula:

$$
u^{\varepsilon}(x) \longrightarrow \bar{u}(x)=b(0) e^{-|x|},
$$

in the uniform norm. Since we have assumed that $b(0)<0$, this function is not the zero function, therefore not the solution (vanishing at infinity) to the one-dimensional version of (120). The function $\bar{u}$ is actually the solution to

$$
\begin{cases}\bar{u}+\left|\bar{u}^{\prime}\right|=0, & \text { for } \quad x \neq 0, \\ \bar{u}(0)=b(0), & \bar{u}(x) \stackrel{|x| \rightarrow \infty}{\longrightarrow} 0\end{cases}
$$

which equation is, in essence, very different from (120). The behavior we have just observed in the onedimensional setting is actually general to all dimensions. We now outline the general argument. 
In the higher dimensional setting, one cannot solve equation (117) explicitly. First, the right notion of solutions should be adopted and, in that setting, it is the notion of viscosity solution. Equation (117) is indeed recognized as a particular instance of the more general first order Hamilton Jacobi equation

$$
H_{\varepsilon}\left(x, u^{\varepsilon}, \nabla u^{\varepsilon}\right)=0
$$

for the particular choice of Hamiltonian function

$$
H_{\varepsilon}(x, u, p)=u+|p|-b(. / \varepsilon) .
$$

General theorems (see e.g. [4]) of the theory of viscosity solutions provide us with a well-posedness result in $W^{1, \infty}$ for that equation. Then, the simple observation

$$
u^{\varepsilon}(0) \leq b(0)=\inf _{\mathbb{R}^{d}} b<0
$$

suffices to show that, when $\varepsilon \rightarrow 0, u^{\varepsilon}$ cannot continuously converge to zero, the unique viscosity solution to (120) in $\mathbb{R}^{d}$. We emphasize again the importance of the sign of the function $b$, more precisely that $\inf _{\mathbb{R}^{d}} b<0$. Otherwise, as in our discussion above on the one-dimensional setting, the limit would be different.

Nevertheless, the statement (124) above is not sufficient to claim that $u^{\varepsilon}$ does not converge to zero in a topology weaker than that of the continuous norm. In order to entirely settle the issue, it is indeed possible, using classical arguments of the theory of viscosity solutions, to show that $u^{\varepsilon}$ continuously converges to $\bar{u}(x)=b(0) e^{-|x|}$, the solution to the $d$-dimensional version of (123), namely

$$
\begin{cases}\bar{u}+|\nabla \bar{u}|=0, & \text { for } \quad x \neq 0 \in \mathbb{R}^{d}, \\ \bar{u}(0)=b(0), \quad \bar{u}(x) \stackrel{|x| \rightarrow \infty}{\longrightarrow} 0\end{cases}
$$

As in the one-dimensional case, we observe that the presence of the local "defect" $b$ has significantly, macroscopically modified the homogenized limit.

Remark 31 Of course the fact the $b$ attains its infimum at the origin does not play any particular role in the above. If (119) does not hold, the limit obtained is $\bar{u}(x)=\left(\inf _{\mathbb{R}^{d}} b\right) e^{-|x|}$. The point is $\inf _{\mathbb{R}^{d}} b<0$. If $b \in L^{p}, p<+\infty$, instead of $b \in \mathcal{D}$, we conclude similarly.

\section{A Technical lemmas}

In this section, we prove the following results, which were used in the proof of Theorem 5.7. In all the following, for any function $f$ that is quasiperiodic with respect to $x^{\prime}=\left(x_{2}, \ldots, x_{d}\right)$, we define

$$
\left\langle|f|^{2}\right\rangle_{\mathbb{R}^{d-1}}=\lim _{R \rightarrow+\infty} \frac{1}{\left|B_{R}\right|} \int_{B_{R}}\left|f\left(x_{1}, x^{\prime}\right)\right|^{2} d x^{\prime} .
$$

Lemma A.1 Assume that $F: \mathbb{R}^{d} \rightarrow \mathbb{R}^{d}$ is smooth, quasiperiodic with respect to the variables $\left(x_{2}, \ldots, x_{d}\right)=$ $x^{\prime}$ and satisfies

$$
\int_{\mathbb{R}}\left\langle|F|^{2}\right\rangle_{\mathbb{R}^{d-1}} d x_{1}<+\infty
$$

where $\left\langle|F|^{2}\right\rangle_{\mathbb{R}^{d-1}}$ is defined by (125). Then, there exists a unique (up to the addition of a constant) $u \in L_{\mathrm{loc}}^{1}$ such that $\nabla u$ is quasiperiodic with respect to $x^{\prime}$ with the same quasiperiod as $F$,

$$
\int_{\mathbb{R}}\left\langle|\nabla u|^{2}\right\rangle_{\mathbb{R}^{d-1}} d x_{1}<+\infty
$$

and

$$
-\Delta u=\operatorname{div}(F)
$$


Moreover, there exists a constant $C$ independent of $F$ such that

$$
\int_{\mathbb{R}}\left\langle|\nabla u|^{2}\right\rangle_{\mathbb{R}^{d-1}} d x_{1} \leq C \int_{\mathbb{R}}\left\langle|F|^{2}\right\rangle_{\mathbb{R}^{d-1}} d x_{1}
$$

If, in addition, $F$ is a trigonometric polynomial in the variables $x^{\prime}$, then $u$ is also a trigonometric polynomial.

Proof: the existence may be proved using the same arguments as for the existence of the corrector in Theorem 5.7. However, it is also possible to deal with it using Fourier series. Indeed, it is known that trigonometric polynomials are dense in the space of quasiperiodic functions equipped with the uniform norm (see for instance [13] or [26]). Hence, it is sufficient to prove the result for $F$ being a trigonometric polynomial:

$$
F(x)=\sum_{k=1}^{K} F_{k}\left(x_{1}\right) e^{2 i \pi \omega_{k} \cdot x^{\prime}},
$$

where for each $k, \omega_{k} \in \mathbb{R}^{d-1}$. As a consequence,

$$
\operatorname{div}(F)=\sum_{k=1}^{K}\left(F_{k}^{\prime}\left(x_{1}\right) \cdot e_{1}+2 i \pi F_{k}\left(x_{1}\right) \cdot \omega_{k}\right) e^{2 i \pi \omega_{k} \cdot x^{\prime}}
$$

Here, by abuse of notation, we define $F_{k}\left(x_{1}\right) \cdot \omega_{k}$ as the scalar product between $F_{k}\left(x_{1}\right) \in \mathbb{R}^{d}$ and $\left(0, \omega_{k}\right) \in \mathbb{R}^{d}$. Now, we look for $u$ as

$$
u(x)=\sum_{k=1}^{K} u_{k}\left(x_{1}\right) e^{2 i \pi \omega_{k} \cdot x^{\prime}},
$$

and compute

$$
-\Delta u(x)=\sum_{k=1}^{K}\left(-u_{k}^{\prime \prime}\left(x_{1}\right)+4 \pi^{2}\left|\omega_{k}\right|^{2} u_{k}\left(x_{1}\right)\right) e^{2 i \pi \omega_{k} \cdot x^{\prime}} .
$$

Hence, by identification, we have

$$
\forall k \in\{1, \ldots, K\}, \quad-u_{k}^{\prime \prime}+4 \pi^{2}\left|\omega_{k}\right|^{2} u_{k}=F_{k}^{\prime}\left(x_{1}\right) \cdot e_{1}+2 i \pi F_{k}\left(x_{1}\right) \cdot \omega_{k} .
$$

If $\omega_{k}=0$, we find $u_{k}^{\prime}=F_{k} \cdot e_{1}$ due to the fact that $u_{k}^{\prime} \in L^{2}(\mathbb{R})$, so that any primitive of $F_{k} \cdot e_{1}$ is a solution. If $\omega_{k} \neq 0$, we define

$$
W_{k}\left(x_{1}\right)=\frac{1}{4 \pi\left|\omega_{k}\right|} e^{-2 \pi\left|\omega_{k}\right|\left|x_{1}\right|},
$$

which is solution to $-W_{k}^{\prime \prime}+4 \pi^{2}\left|\omega_{k}\right|^{2} W_{k}=\delta_{0}$. Hence, $u_{k}=W_{k} *\left(F_{k}^{\prime} \cdot e_{1}+2 i \pi F_{k} \cdot \omega_{k}\right)$ This convolution product exists since $F_{k}^{\prime} \cdot e_{1}+2 i \pi F_{k} \cdot \omega_{k} \in H^{-1}(\mathbb{R})$ and $W_{k} \in H^{1}(\mathbb{R})$. This reads

$$
u_{k}\left(x_{1}\right)=\int_{\mathbb{R}} W_{k}\left(x_{1}-y_{1}\right) 2 i \pi F_{k}\left(y_{1}\right) \cdot \omega_{k} d y_{1}+\int_{\mathbb{R}} W_{k}^{\prime}\left(x_{1}-y_{1}\right) F_{k}\left(y_{1}\right) \cdot e_{1} d y_{1}
$$

We differentiate this equality, and use the differential equation satisfied by $W_{k}$, finding

$$
u_{k}^{\prime}\left(x_{1}\right)=-F_{k}\left(x_{1}\right) \cdot e_{1}+\int_{\mathbb{R}} 4 \pi^{2}\left|\omega_{k}\right|^{2} W_{k}\left(x_{1}-y_{1}\right) d y_{1}+\int_{\mathbb{R}} 2 i \pi F_{k}\left(y_{1}\right) \cdot \omega_{k} W_{k}^{\prime}\left(x_{1}-y_{1}\right) d y_{1} .
$$

Hence, applying Young's inequality and (130),

$$
\begin{aligned}
& \left\|u_{k}^{\prime}\right\|_{L^{2}(\mathbb{R})} \leq\left\|F_{k}\left(x_{1}\right)\right\|_{L^{2}(\mathbb{R})}+2\left\|4 \pi^{2}\left|\omega_{k}\right|^{2} W_{k} * F_{k}\right\|_{L^{2}(\mathbb{R})} \\
& \quad \leq\left\|F_{k}\left(x_{1}\right)\right\|_{L^{2}(\mathbb{R})}+2\left\|F_{k}\right\|_{L^{2}(\mathbb{R})}\left\|4 \pi^{2}\left|\omega_{k}\right|^{2} W_{k}\right\|_{L^{1}(\mathbb{R})}=3\left\|F_{k}\left(x_{1}\right)\right\|_{L^{2}(\mathbb{R})} .
\end{aligned}
$$

This proves that, both in the case $\omega_{k}=0$ and in the case $\omega_{k} \neq 0, u_{k}^{\prime} \in L^{2}$. A similar argument allows to prove that

$$
\left\|2 \pi \omega_{k} u_{k}\right\|_{L^{2}(\mathbb{R})} \leq\left\|F_{k}\right\|_{L^{2}(\mathbb{R})} .
$$


Hence, $u$ defined by (129) does satisfy (128). Finally, it is clear that $u$ is a solution of (127).

Bessel inequality allows to use a density argument and conclude the proof of existence in the general case.

Finally, uniqueness may be proved as follows: if $\nabla u$ satisfies (126), then it can be decomposed in Fourier series. The above analysis shows that it is equal to the limit obtained in the above density argument.

Lemma A.2 Let $u \in H_{\mathrm{loc}}^{1}\left(\mathbb{R}^{d}\right)$ be such that $\nabla u$ is quasiperiodic in $x^{\prime}=\left(x_{2}, \ldots, x_{d}\right)$, and

$$
\int_{\mathbb{R}}\left\langle|\nabla u|^{2}\right\rangle_{\mathbb{R}^{d-1}} d x_{1}<+\infty
$$

where $\left\langle|\nabla u|^{2}\right\rangle_{\mathbb{R}^{d-1}}$ is defined by (125). Then, there exists a sequence $\left(u_{n}\right)_{n \in \mathbb{N}}$ such that $u_{n} \in C^{\infty}\left(\mathbb{R}^{d}\right), \nabla u_{n}$ is quasiperiodic in $x^{\prime}$ (with same quasiperiods as $u$ ), $u_{n}$ has compact support with respect to $x_{1}$, and

$$
\int_{\mathbb{R}}\left\langle\left|\nabla u-\nabla u_{n}\right|^{2}\right\rangle_{\mathbb{R}^{d-1}} d x_{1} \underset{n \rightarrow+\infty}{\longrightarrow} 0 .
$$

Remark 32 As it is clear in the proof below, it is possible to assume that the size of the support of $u_{n}$ in $x_{1}$ is bounded uniformly with respect to $x^{\prime}$, that is,

$$
\forall n \in \mathbb{N}, \quad \exists R_{n}>0, \quad \text { such that } \operatorname{supp} u_{n} \subset\left\{x \in \mathbb{R}^{d}, \quad\left|x_{1}\right| \leq R_{n}\right\} .
$$

Proof: Here again, using a density argument, we can assume that $u$ is a trigonometric polynomial in the variables $x^{\prime}$. We define a smooth cut-off $\chi_{n} \in C^{\infty}(\mathbb{R})$ such that:

$$
\chi_{n} \geq 0, \quad \chi_{n}\left(x_{1}\right)=1 \text { if }\left|x_{1}\right| \leq n, \quad \chi_{n}\left(x_{1}\right)=0 \text { if }\left|x_{1}\right| \geq n+1, \quad\left\|\chi_{n}^{\prime}\right\|_{L^{\infty}} \leq 2 .
$$

Applying Lemma A.1, we know that there exists $v_{n} \in H_{\text {loc }}^{1}\left(\mathbb{R}^{d}\right)$ such that $v_{n}$ is quasiperiodic in $x^{\prime}=$ $\left(x_{2}, \ldots, x_{n}\right)$ (with same quasiperiod as $u$ ),

$$
-\Delta v_{n}=-\operatorname{div}\left(\chi_{n} \nabla u\right),
$$

and

$$
\int_{\mathbb{R}}\left\langle\left|\nabla v_{n}\right|^{2}\right\rangle_{\mathbb{R}^{d-1}} d x_{1}<+\infty
$$

Still applying Lemma A.1, we have

$$
\int_{\mathbb{R}}\left\langle\left|\nabla v_{n}-\nabla u\right|^{2}\right\rangle d x_{1} \leq C \int_{\mathbb{R}}\left\langle\left|\chi_{n}-1\right|^{2}|\nabla u|^{2}\right\rangle d x_{1} \leq C \int_{\left|x_{1}\right| \geq n}\left\langle\left|\chi_{n}-1\right|^{2}|\nabla u|^{2}\right\rangle d x_{1} \underset{n \rightarrow+\infty}{\longrightarrow} 0 .
$$

The last estimate is a simple application of the dominated convergence theorem. In addition, we know that $v_{n}$ itself is a trigonometric polynomial. We thus write

$$
v_{n}(x)=\sum_{k=1}^{K} v_{n, k}\left(x_{1}\right) e^{2 i \pi \omega_{k} \cdot x^{\prime}}
$$

where, for each $k, \omega_{k} \in \mathbb{R}^{d-1}$, and, according to (131),

$$
\int_{\mathbb{R}}\left|v_{n, k}^{\prime}\right|^{2}+4 \pi^{2}\left|\omega_{k}\right|\left|v_{n, k}\right|^{2}<+\infty
$$

hence $v_{n, k} \in H^{1}(\mathbb{R})$, except possibly if $\omega_{k}=0$. But in such a case, $v_{n, k}^{\prime} \in L^{2}(\mathbb{R})$, and $v_{n, k}^{\prime \prime}\left(x_{1}\right)=0$ if $\left|x_{1}\right| \geq n+1$, which implies that $v_{n, k}$ is a constant at infinity. Hence it is bounded. In the case $\omega_{k} \neq 0$, $v_{n, k} \in H^{1}\left(\mathbb{R}^{d}\right) \subset L^{\infty}(\mathbb{R})$, hence it is bounded too. This proves that $v_{n} \in L^{\infty}\left(\mathbb{R}^{d}\right)$. Finally, we define a new cut-off function $\phi_{p} \in C^{\infty}(\mathbb{R})$ such that

$$
\phi_{p} \geq 0, \quad \phi_{p}\left(x_{1}\right)=1 \text { if }\left|x_{1}\right| \leq p, \quad \phi_{p}\left(x_{1}\right)=0 \text { if }\left|x_{1}\right| \geq 2 p, \quad\left\|\phi_{p}^{\prime}\right\|_{L^{\infty}} \leq \frac{2}{p}
$$


Setting

$$
u_{n, p}(x)=\phi_{p}\left(x_{1}\right) v_{n}(x),
$$

we have that $u_{n, p}$ is a trigonometric polynomial, hence is quasiperiodic, and has compact support in $x_{1}$. Further, we have, for any $p \geq n+1$,

$$
\nabla u_{n, p}-\nabla v_{n}=\left(\phi_{p}\left(x_{1}\right)-1\right) \nabla v_{n}+\phi_{p}^{\prime}\left(x_{1}\right) v_{n} e_{1}=\phi_{p}^{\prime}\left(x_{1}\right) v_{n} e_{1}
$$

Hence,

$$
\int_{\mathbb{R}}\left\langle\left|\nabla u_{n, p}-\nabla v_{n}\right|^{2}\right\rangle_{\mathbb{R}^{d-1}} d x_{1} \leq \frac{4}{p^{2}} \int_{p \leq\left|x_{1}\right| \leq 2 p}\left\|v_{n}\right\|_{L^{\infty}}^{2} d x_{1}=\frac{4\left\|v_{n}\right\|_{L^{\infty}}^{2}}{p}
$$

Combining this inequality with (132), we let first $p$, then $n$, tend to infinity, and we get the result.

Lemma A.3 Let $F: \mathbb{R}^{+} \rightarrow \mathbb{R}^{+}$be a $C^{1}$ non-increasing function such that there exists $C_{1}, C_{2}>0$ satisfying

$$
\forall R \geq 1, \quad F(R) \leq C_{1} \sqrt{-F^{\prime}(R)}+\frac{C_{2}}{R} .
$$

Then there exists a constant $C>0$ such that

$$
\forall R \geq 0, \quad F(R) \leq \frac{C}{R}
$$

Proof: we first note that, multiplying $F$ by some constant if necessary, we may assume that $C_{1}=1$. Then, we fix $\gamma \geq 3$ large enough to have

$$
\forall r \in[0,1], \quad F(r)<\frac{\gamma}{r}, \quad \text { and } \quad \gamma<\left(\gamma-C_{2}\right)^{2} .
$$

We then define two subsets of $\mathbb{R}^{+}$:

$$
\begin{aligned}
& A=\left\{r \in \mathbb{R}^{+}, \quad F(r) \leq \frac{\gamma}{r}\right\}, \\
& B=\left\{r \in \mathbb{R}^{+}, \quad F(r)>\frac{\gamma}{r}\right\} .
\end{aligned}
$$

The definition of $\gamma$ implies that $A$ is not empty. We first claim that $B$ has no bounded connected component. Indeed, if $B_{0}$ is such a set, then $B_{0}=\left(R_{1}, R_{2}\right)$, with $1<R_{1}<R_{2}$ and $R_{1} F\left(R_{1}\right)=R_{2} F\left(R_{2}\right)=\gamma$. Moreover, we have, according to (133) and the definition of $B, \forall r \in B_{0}, \quad \sqrt{-F^{\prime}(r)} \geq F(r)-\frac{C_{2}}{r} \geq \frac{\gamma-C_{2}}{r}$. This implies $-F^{\prime}(r) \geq\left(\gamma-C_{2}\right)^{2} / r^{2}$, hence

$$
F\left(R_{1}\right)-F\left(R_{2}\right) \geq\left(\gamma-C_{2}\right)^{2} \int_{R_{1}}^{R_{2}} \frac{d r}{r^{2}}=\left(\gamma-C_{2}\right)^{2}\left(\frac{1}{R_{1}}-\frac{1}{R_{2}}\right)
$$

This implies $\gamma \geq\left(\gamma-C_{2}\right)^{2}$, which is in contradiction with (135). Hence, $B$ is of the form $B=\left(R_{1},+\infty\right)$, for some $R_{1} \geq 1$. Here again, we have, for any $r \in B,-F^{\prime}(r) \geq\left(\gamma-C_{2}\right)^{2} / r^{2}$, hence

$$
F\left(R_{1}\right)-F(r) \geq\left(\gamma-C_{2}\right)^{2}\left(\frac{1}{R_{1}}-\frac{1}{r}\right) .
$$

Letting $r \rightarrow+\infty$, we find $F\left(R_{1}\right) \geq\left(\gamma-C_{2}\right)^{2} / R_{1}$, which implies $\gamma \geq\left(\gamma-C_{2}\right)^{2}$. This is a contradiction again.

We deduce that $B=\emptyset$, so that $A=\mathbb{R}^{+}$, thereby proving (134).

Lemma A.4 Consider $\varphi: \mathbb{R}^{+} \rightarrow \mathbb{R}$ such that $\varphi^{\prime} \in L^{2}\left(\mathbb{R}^{+}\right)$. Assume that there exists a constant $C>0$ satisfying

$$
\forall T \geq 0, \quad \int_{T}^{+\infty}\left|\varphi^{\prime}(t)\right|^{2} d t \leq \frac{C}{T^{2}}
$$

Then, $\varphi \in L^{\infty}\left(\mathbb{R}^{+}\right)$. 
Proof: The fact that $\varphi^{\prime} \in L^{2}$ clearly implies that $\varphi \in L_{\text {loc }}^{\infty}\left(\mathbb{R}^{+}\right)$, so that we only need to prove that $\varphi$ is bounded at infinity. In order to do so, we write

$$
\forall x \in \mathbb{R}^{+}, \quad|\varphi(2 x)-\varphi(x)|=\left|\int_{x}^{2 x} \varphi^{\prime}(t) d t\right| \leq \sqrt{x}\left(\int_{x}^{2 x}\left|\varphi^{\prime}(t)\right|^{2} d t\right)^{1 / 2} .
$$

Hence, applying (136), we have

$$
\forall x \in \mathbb{R}^{+}, \quad|\varphi(2 x)-\varphi(x)|=\frac{C}{\sqrt{x}} .
$$

Now, for $x \in \mathbb{R}^{+}, x \neq 0$, let $n \in \mathbb{N}$ be such that $2^{n} \leq x<2^{n+1}$. We write

$$
\varphi(x)=\sum_{k=0}^{n}\left[\varphi\left(\frac{x}{2^{k}}\right)-\varphi\left(\frac{x}{2^{k+1}}\right)\right]+\varphi\left(\frac{x}{2^{n+1}}\right) .
$$

Applying (137), the $k$-th term in the sum is bounded by $C \sqrt{2^{k+1} / x}$, whence

$$
|\varphi(x)| \leq \frac{C}{\sqrt{x}} \sum_{k=0}^{n} 2^{\frac{k+1}{2}}+\left|\varphi\left(\frac{x}{2^{n+1}}\right)\right| \leq \frac{C}{\sqrt{x}} \sqrt{2} \frac{2^{\frac{n+1}{2}}-1}{\sqrt{2}-1}+\|\varphi\|_{L^{\infty}(0,1)} \leq C \frac{\sqrt{2}}{\sqrt{2}-1} \sqrt{\frac{2^{n+1}}{x}}+\|\varphi\|_{L^{\infty}(0,1)} .
$$

According to the definition of $n$, this implies that the right-hand side is bounded independently of $x$. Hence, (136) is proved.

\section{Acknowledgement}

The work of the second author is partly supported by ONR under Grant N00014-12-1-0383 and by EOARD under Grant FA8655-13-1-3061.

\section{References}

[1] L. Ambrosio, H. Frid, Multiscale Young measures in almost periodic homogenization and applications. Arch. Ration. Mech. Anal. 192 (2009), no. 1, 37-85.

[2] L. Ambrosio, N. Fusco, D. Pallara, Functions of bounded variation and free discontinuity problems. Oxford Mathematical Monographs. The Clarendon Press, Oxford University Press, New York, 2000.

[3] M. Avellaneda, F. H. Lin, Compactness methods in the theory of homogenization, Communications on Pure and Applied Mathematics 40, no. 6, pp 803 - 847, 1987.

[4] G. Barles, Solutions de viscosité des équations de Hamilton-Jacobi, Mathématiques et Applications, Springer, 1994.

[5] A. Bensoussan, J. L. Lions, G. Papanicolaou, Asymptotic analysis for periodic structures, Studies in Mathematics and its Applications, 5. North-Holland Publishing Co., Amsterdam-New York, 1978.

[6] J. Bergh, J. Löfström, Interpolation spaces. An introduction, Grundlehren der mathematischen Wissenschaften, Vol. 223, Springer, Berlin Heidelberg, 1976.

[7] X. Blanc, C. Le Bris, P.-L. Lions, A definition of the ground state energy for systems composed of infinitely many particles, Communications in P.D.E., Vol 28, no 1-2, pp 439-475, 2003.

[8] X. Blanc, C. Le Bris, P.-L. Lions, Une variante de la théorie de l'homogénéisation stochastique des opérateurs elliptiques, [A variant of stochastic homogenization theory for elliptic operators], Note aux Comptes Rendus de l'Académie des Sciences, t. 343, Série 1, p 711-724, 2006.

[9] X. Blanc, C. Le Bris, P.-L. Lions, Stochastic homogenization and random lattices, Journal de Mathématiques Pures et Appliquées, 88, pp 34-63, 2007. 
[10] X. Blanc, C. Le Bris, P.-L. Lions, A possible homogenization approach for the numerical simulation of periodic microstructures with defects, Milan Journal of Mathematics, Vol. 80, pp 351-367, 2012.

[11] X. Blanc, C. Le Bris, P.-L. Lions, Profils locaux et problèmes elliptiques à plusieurs échelles avec défauts [Local profiles and elliptic problems at different scales with defects], C. R. Acad. Sci. Paris, Ser. I 353 (2015) 203-208.

[12] X. Blanc, F. Legoll, A. Anantharaman, Asymptotic behaviour of Green functions of divergence form operators with periodic coefficients, Applied Mathematics Research Express, (1), pp 79-101 (2013).

[13] A. Braides, V. Chiado Piat, and A. Defranceschi. Homogenization of almost periodic monotone operators. Annales de l'Institut Henri Poincare (C) Non Linear Analysis, 9(4):399-432, 1992.

[14] G. Dolzmann, S. Müller, Estimates for Green's matrices of elliptic systems by $L^{p}$ theory, Manuscripta Mathematica 88, no. 2, pp 261-273, 1995.

[15] Y. Efendiev, T. Hou, Multiscale Finite Element method, Theory and applications. Surveys and Tutorials in the Applied Mathematical Sciences, 4. Springer, New York, 2009.

[16] S. R. Finch, Mathematical constants, in Encyclopedia of Mathematics and its Applications, 94, Cambridge University Press, Cambridge, 2003.

[17] M. Giaquinta, Multiple integrals in the calculus of variations and nonlinear elliptic systems, Princeton University Press, 1983.

[18] D. Gilbarg, N. S. Trudinger, Elliptic partial differential equations of second order. Reprint of the 1998 edition. Classics in Mathematics. Springer-Verlag, Berlin, 2001.

[19] A. Gloria, D. Marahrens, Annealed estimates on the Green functions and uncertainty quantification, arXiv:1409.0569, 2014.

[20] A. Gloria, Z. Habibi, Reduction of the resonance error in numerical homogenization II: correctors, extrapolation and spectral theory, 2014. https://hal.inria.fr/hal-00933234v2

[21] M. Grüter, K. O. Widman, The Green function for uniformly elliptic equations, Manuscripta Mathematica 37, no. 3, pp 303 - 342, 1982.

[22] V. V. Jikov, S. M. Kozlov, O. A. Oleinik, Homogenization of differential operators and integral functionals, Springer-Verlag, 1994.

[23] C. Kenig and W. M. Ni, On the elliptic equation $L u-k+K \exp [2 u]=0$, Ann. Scuola Norm. Super. Pisa (IV) 12:191-224, 1985.

[24] C. Kenig, H. Koch, J. Pipher, T. Toro, A new approach to absolute continuity of elliptic measure, with applications to non-symmetric equations, Advances in Mathematics, 153, 2:231-298, 2000.

[25] S. M. Kozlov, Averaging of differential operators with almost periodic rapidly oscillating coefficients, Mat. Sb. (N.S.), 107(149)(2):199-217, 317, 1978.

[26] B. M. Levitan and V. V. Zhikov. Almost periodic functions and differential equations. Cambridge University Press, Cambridge, 1982.

[27] P.-L. Lions, On the Schwarz alternating method. III. A variant for nonoverlapping subdomains. Third International Symposium on Domain Decomposition Methods for Partial Differential Equations (Houston, TX, 1989), 202-223, SIAM, Philadelphia, PA, 1990.

[28] P.-L. Lions, Lectures 2013-2014 at Collège de France, video recording available at www. college-de-france.fr/site/pierre-louis-lions.

[29] P.-L. Lions, P. Souganidis, in preparation. 
[30] N. G. Meyers, An $L^{p}$-estimate for the gradient of solutions of second order elliptic divergence form equations, Ann. Scuola Norm. Sup. Pisa. 17, pp 189-206, 1963.

[31] J. Moser, On Harnack's theorem for elliptic differential equations, Communications on Pure and Applied Mathematics 14, no. 3, pp 577-591, 1961.

[32] O. A. Oleunik and V. V. Zhikov. On the homogenization of elliptic operators with almost-periodic coefficients. In Proceedings of the international conference on partial differential equations dedicated to Luigi Amerio on his 70th birthday (Milan/Como, 1982), volume 52, pages 149-166 (1985), 1982.

[33] L. C. Piccinini, S. Spagnolo, On the Hölder continuity of solutions of second order elliptic equations in two variables. Ann. Scuola Norm. Sup. Pisa (3) 26 (1972), 391-402.

[34] L. Tartar, An introduction to Sobolev spaces and interpolation spaces, Lecture Notes of the Unione Matematica Italiana 3, Berlin, Springer, 2007.

[35] W.P. Ziemer, Weakly differentiable functions, Springer, New-York, 1989. 